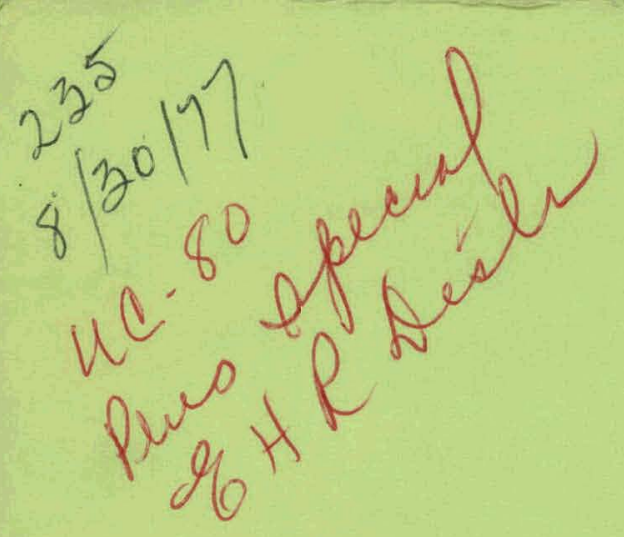

\title{
Operating History
}

\section{U.S. Central Station Nuclear Power Plants}

Energy Research \& Development Administration Division of Nuclear Research \& Applications 9503532 


\section{DISCLAIMER}

This report was prepared as an account of work sponsored by an agency of the United States Government. Neither the United States Government nor any agency Thereof, nor any of their employees, makes any warranty, express or implied, or assumes any legal liability or responsibility for the accuracy, completeness, or usefulness of any information, apparatus, product, or process disclosed, or represents that its use would not infringe privately owned rights. Reference herein to any specific commercial product, process, or service by trade name, trademark, manufacturer, or otherwise does not necessarily constitute or imply its endorsement, recommendation, or favoring by the United States Government or any agency thereof. The views and opinions of authors expressed herein do not necessarily state or reflect those of the United States Government or any agency thereof. 


\section{DISCLAIMER}

Portions of this document may be illegible in electronic image products. Images are produced from the best available original document. 


\section{NOTICE}

Tiis report was prepared as an account of work sponsored by the United States Government. Neither the United States nor the United States Energy Research and Development Administration, nor any of their employees, nor any of their contractors, subcontractors, or their employees, makes any warranty, express or implied, or assumes any legal liability or responsibility for the accuracy, coinpleteness or usefulness of any information, apparatus, product or process disclosed, or represents that its use would not infringe privately owned rights.

Avallable from:

National Technical Information Service (NTIS)

U.S. Department of Commerce

5285 Port Royal Road

Springfield, Virginia 22161

Price:

Printed Copy:

$\$ 5.00$

Microfiche:

$\$ 3.00$ 


\section{OPERAT ING HISTORY}

$\mathrm{OF}$

U.S. CENTRAL STATION NUCLEAR POWER PLANTS

The information assembled in this booklet highlights the operating history of U.S. Central Station nuclear power plants through December 31,1975 . The information presented herein is based on data furnished by the operating electric utilities and was prepared by the Division of Nuclear Research and Applications of ...the U.S. Energy Research and Development Administration (ERDA). The information is presented in the form of statistical tables and computer printouts of major shutdown periods for each nuclear unit. Starting with 1976, the information is based on data prepared by the utilities for the Nuclear Regulatory Commission (NRC), whereas previously the data was submitted separately to ERDA and the former U.S. Atomic Energy Commission. For 1976 the capacity factor data for each unit is presented both on the basis of its net design electrical rating and its net maximum dependable capacity, as reported by the operating utility to the Nuclear Regulatory

Commission. 
CONTENTS

TABLES

$\underline{\text { Page }}$

1. Selected Operating Statistics - 1976

2. Generation of Thermal Energy by Central Station Nuclear Power Plants - Gross Thermal

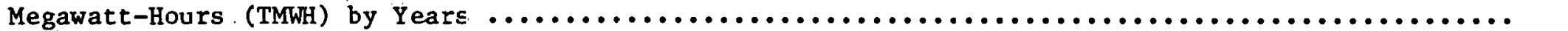

3. Generation of Electricity by Central Station Nuclear Power Plants - Gross Electrical

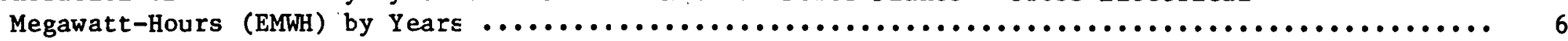

4. Generation of Electricity by Central Station Nuclear Power Plants - Net Electrical

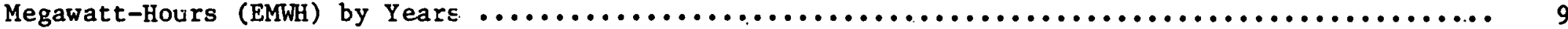

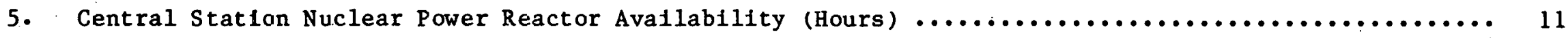

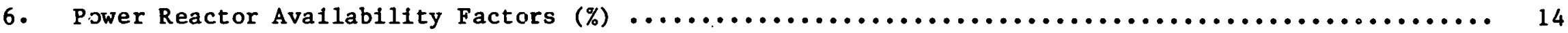

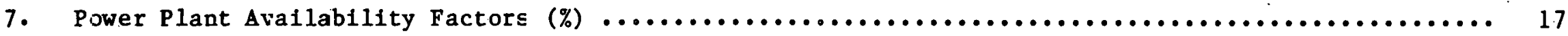

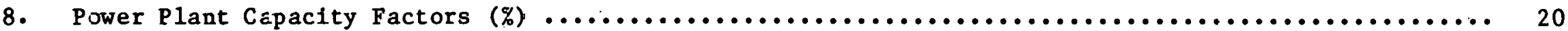

\section{HISTORY OF MAJOR SHUTDOWNS - OPERABLE PLANTS}

Arkansas Nuclear One, Unit \#1

Duane Arnold Energy Center, Unit $\|_{1}$

Beaver Valley, Unit \#1 


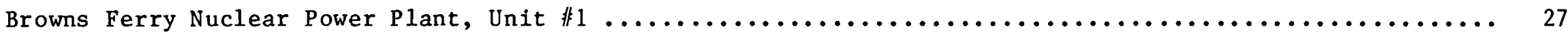

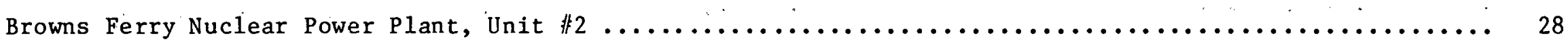

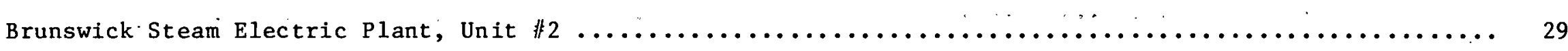

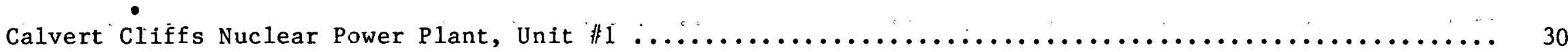

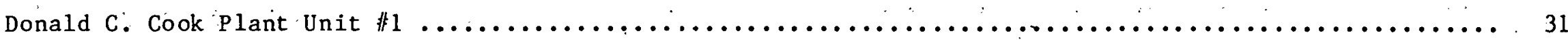

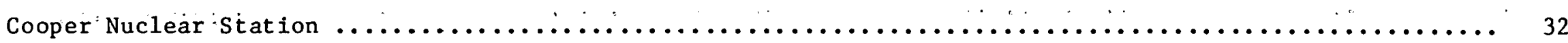

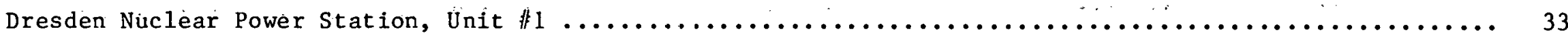

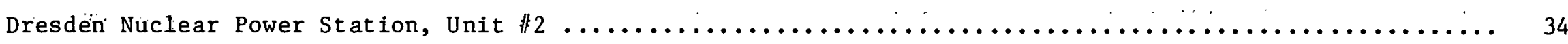

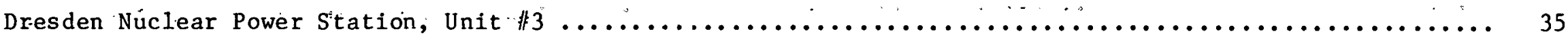

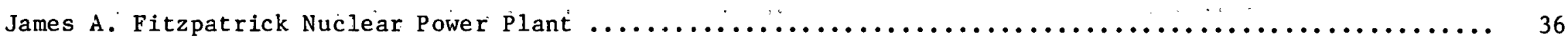

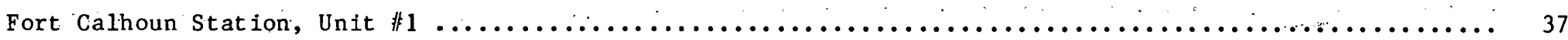

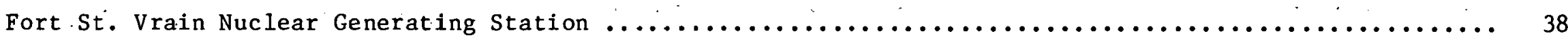

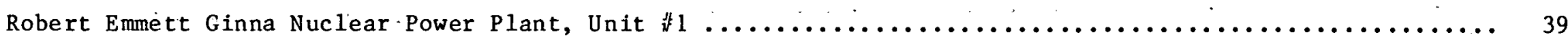

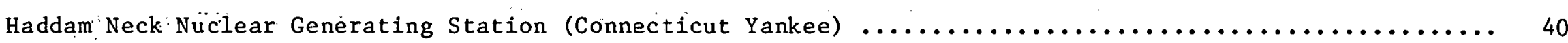

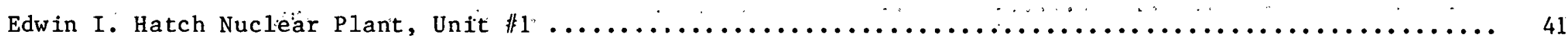

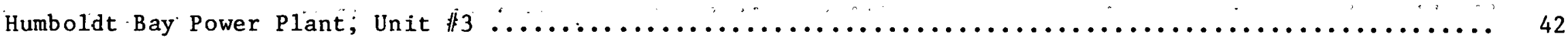


Indian Point Station, Unit 11

Indian Point Station, Unit $\left.\right|_{2}$

Indian Point Station, Unit \#3 .

Kewaunee Nuclear Power Plant, Unit $\neq 1$

LaCrosse (Genoa) Kuclear Generating Station

Maine Yankee Atomic Power Plant

Millstone Nuclear Power Station, Unit \#1

Millstone Nuclear Power Station, Unit 非

-

Monticello Nuclear Generating Plant

...............

Nine Mile Point Nuclear Station, Unzt \#1

Oconee Nuclear Station, Unit $\|_{1}$

\#1

Oconee Nuclear Station, Unit \#2

Oconee Nuclear Station, Unit 非

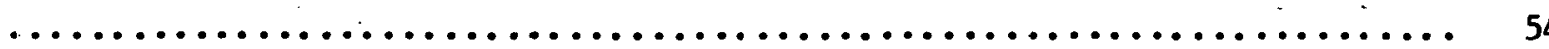

Oyster Creek Nuclear Power Plant, Unit \#1

Palisades Plant

Peach-Bottom Atomfc Power Station; tnit $\#_{2}$

Peach Bottom Atomic Power Station, lnit 非 


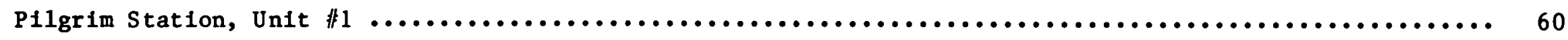

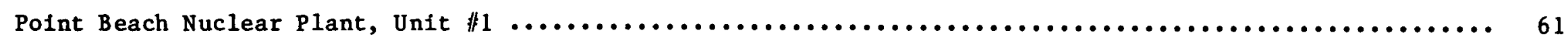

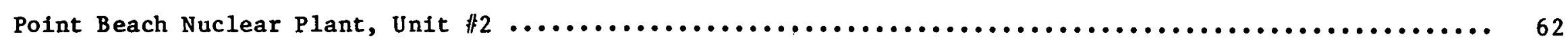

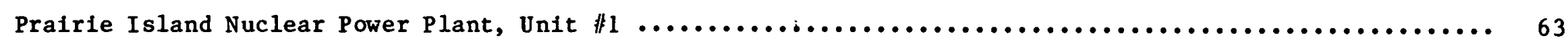

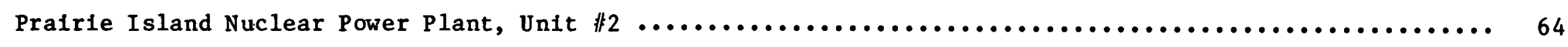

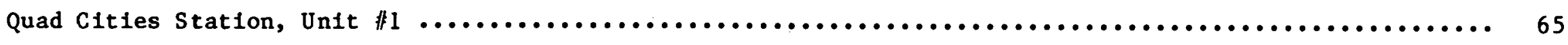

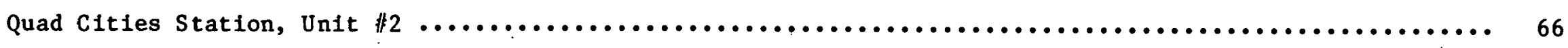

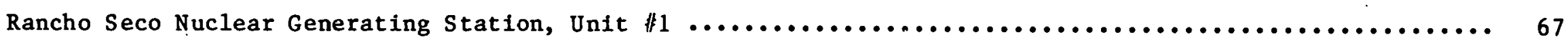

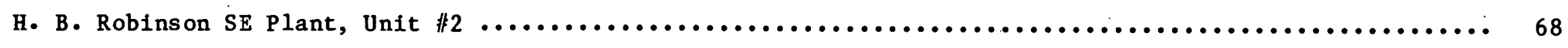

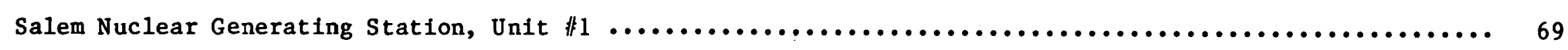

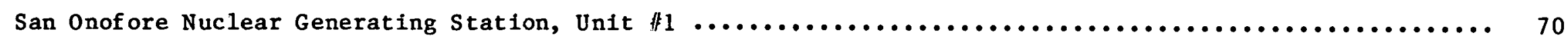

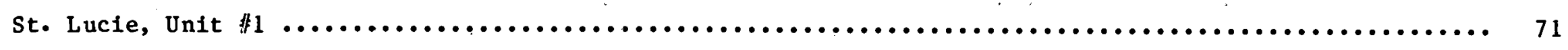

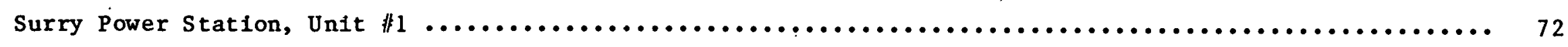

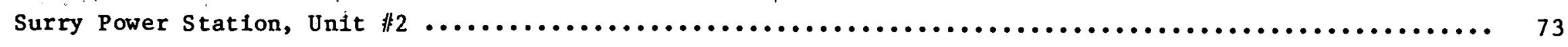

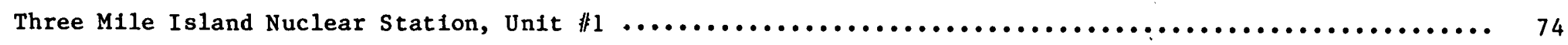

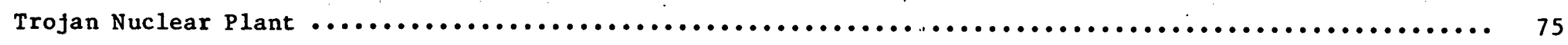


Turkey Point Station, Unit \#3

Turkey Point Station, Unit \#4

Vermont Yankee Generating Station

Zion Station, Unit 非 1 


\section{TA B LES}


TABLE 1 - SELECTEC OPERATINE STATISTICS 1976

FERFCRNANCE INCICATCFS

\begin{tabular}{|c|c|c|c|c|c|c|c|c|c|}
\hline $\begin{array}{c}\text { CESIGN } \\
\text { CAPACITY } \\
\text { (NWE NET) }\end{array}$ & $\begin{array}{c}\text { INI I'IAL } \\
\text { CRITICALITY }\end{array}$ & $\begin{array}{c}\text { FIRST } \\
\text { ELECTR ICITY }\end{array}$ & $\begin{array}{l}\text { ELECTFICAL } \\
\text { (MWH NET) }\end{array}$ & $\begin{array}{c}\text { TRERNAL } \\
\text { (MWH GROSS) }\end{array}$ & $\begin{array}{c}\text { CFIT ICALITY } \\
\text { HCURS }\end{array}$ & $\begin{array}{l}\text { AVAIL } \\
\text { FAC }(\Phi)\end{array}$ & $\begin{array}{l}\text { AVAIL } \\
F \triangle C(q)\end{array}$ & $\begin{array}{l}\text { CESICN } \\
\text { CAPACIT) } \\
\text { FAC }(\&)\end{array}$ & $\begin{array}{l}\text { MAX D DE } \\
\text { CAPACIT } \\
\text { FAC }(\%)\end{array}$ \\
\hline $\begin{array}{r}90.0 \\
200.0 \\
175.0 \\
265.0 \\
72.0 \\
63.0 \\
850.0 \\
430.0 \\
575.0 \\
50.0 \\
650.0 \\
610.0 \\
490.0 \\
809.0 \\
712.0 \\
652.0 \\
497.0 \\
545.0 \\
809.0 \\
668.0 \\
789.0 \\
514.0 \\
785.0 \\
497.0 \\
655.0 \\
822.0 \\
693.0 \\
79 C .0 \\
822.0 \\
887.0 \\
873.0 \\
693.0 \\
1, C 40.0 \\
1, C 65.0 \\
457.0 \\
1, C 65.0 \\
887.0 \\
530.0 \\
1,040.0 \\
330.0 \\
778.0\end{array}$ & $\begin{array}{l}12 / 57 \\
10 / 59 \\
68 / 60 \\
08 / 62 \\
09 / 62 \\
02 / 63 \\
12 / 63 \\
06 / 67 \\
67 / 67 \\
07 / 67 \\
05 / 69 \\
09 / 69 \\
11 / 69 \\
01 / 70 \\
09 / 70 \\
10 / 70 \\
11 / 70 \\
12 / 70 \\
01 / 71 \\
05 / 71 \\
10 / 71 \\
03 / 72 \\
04 / 72 \\
05 / 72 \\
66 / 772 \\
07 / 72 \\
10 / 72 \\
10 / 72 \\
03 / 73 \\
04 / 73 \\
05 / 73 \\
06 / 73 \\
06 / 73 \\
08 / 72 \\
08 / 73 \\
09 / 73 \\
11 / 72 \\
12 / 73 \\
12 / 73 \\
01 / 74 \\
02 / 74\end{array}$ & 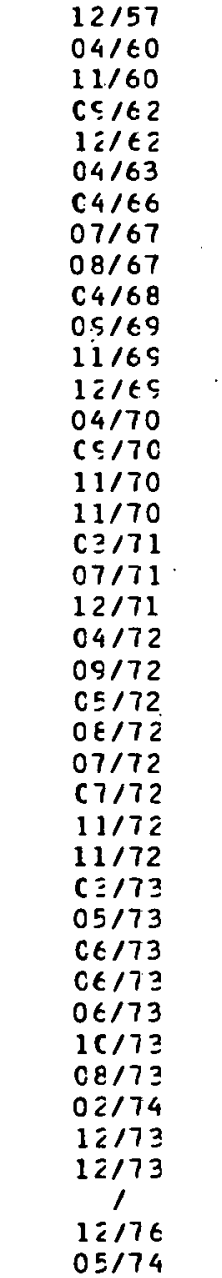 & 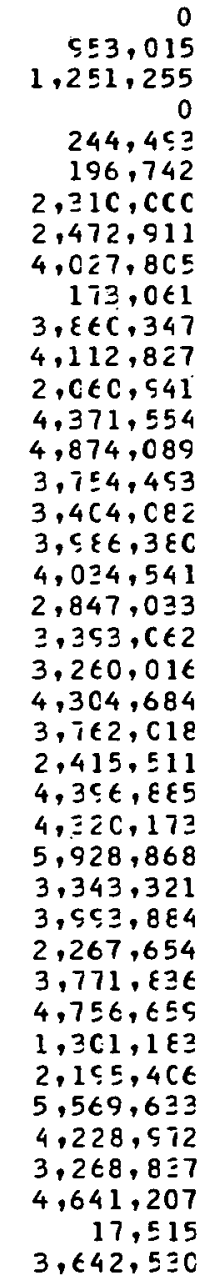 & 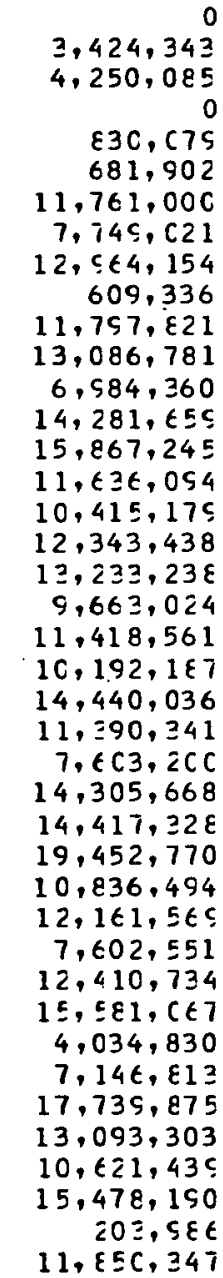 & $\begin{array}{r}0 \\
7,534 \\
7,598 \\
0 \\
4,515 \\
4,111 \\
3,351 \\
6,253 \\
7,587 \\
4,372 \\
7,627 \\
7,887 \\
5,301 \\
6,874 \\
7,579 \\
6,853 \\
7,444 \\
8,228 \\
7,264 \\
5,184 \\
5,548 \\
6,54 C \\
7,331 \\
8,665 \\
5,463 \\
6,058 \\
6,76 C \\
8,256 \\
4,685 \\
5,344 \\
3,247 \\
6,676 \\
5,643 \\
2,335 \\
6,2228 \\
6,147 \\
5,668 \\
6,545 \\
6,556 \\
1,803 \\
6,756\end{array}$ & 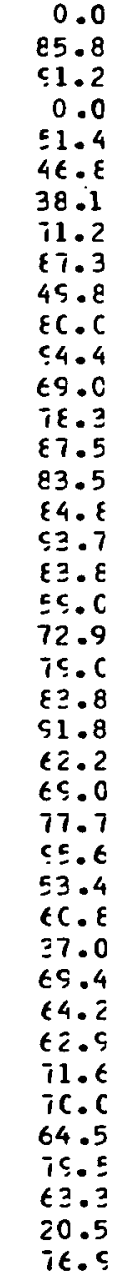 & $\begin{array}{r}0.0 \\
84.2 \\
85.8 \\
0 . C \\
5 C .1 \\
46.4 \\
38.0 \\
7 C .5 \\
82.5 \\
47.4 \\
75.3 \\
88.2 \\
58.3 \\
75.5 \\
84.7 \\
76.1 \\
82.7 \\
91.5 \\
82.4 \\
55.2 \\
64.9 \\
77.1 \\
81.3 \\
90.7 \\
6 C .7 \\
68.5 \\
75.5 \\
55 . C \\
52.3 \\
57.3 \\
34.8 \\
66.4 \\
62.1 \\
34.8 \\
65.5 \\
68.3 \\
62.5 \\
77.4 \\
61.8 \\
75 / A \\
75.5\end{array}$ & 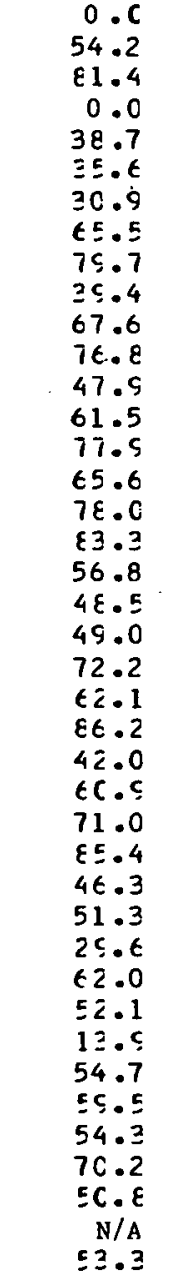 & 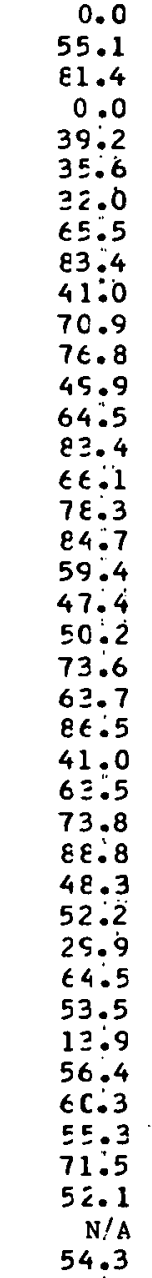 \\
\hline
\end{tabular}


TABLE 1 - SELECTEC OPERATINE STATISTICS ISTE

FERFCFMANCE INCICATORS

\begin{tabular}{|c|c|c|c|c|c|c|c|c|c|c|}
\hline 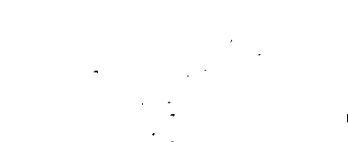 & $\begin{array}{l}\text { CESICN } \\
\text { CAFACITY } \\
\text { (MHE NET) }\end{array}$ & $\begin{array}{c}\text { INITIAL } \\
\text { CRITICALITY }\end{array}$ & $\begin{array}{c}\text { FIRST } \\
\text { ELECTRICITY }\end{array}$ & $\begin{array}{l}\text { ELECTRICAL } \\
\text { (MWt NET) }\end{array}$ & $\begin{array}{c}\text { THERMAL } \\
\text { (MWH GROSS) }\end{array}$ & $\begin{array}{l}\text {... . REACTOH } \\
\text { CRITICALITY } \\
\text { HOURS }\end{array}$ & $\begin{array}{l}\text { A IAIL } \\
\text { FAC }(q)\end{array}$ & $\begin{array}{l}\ldots \ldots \\
A V A I L \\
F A C(x)\end{array}$ & $\begin{array}{r}\text {. F FLANT } \\
\text { [EESICN } \\
\text { CAFACITY } \\
\text { FAC (q) }\end{array}$ & $\begin{array}{l}\text { MAX DEP } \\
\text { CAPACITY } \\
\text { FACI }\end{array}$ \\
\hline 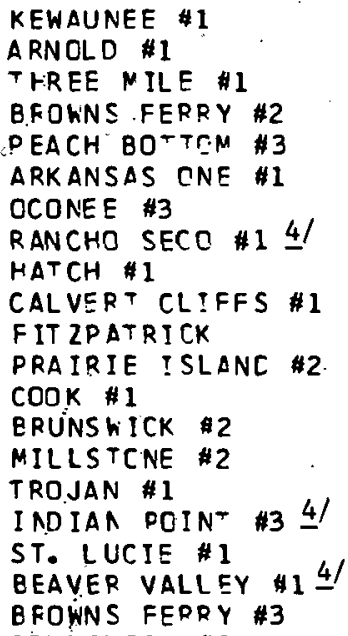 & $\begin{array}{r}535.0 \\
538.0 \\
819.0 \\
1,065.0 \\
1,065.0 \\
850.0 \\
887.0 \\
513.0 \\
786.0 \\
.845 .0 \\
821.0 \\
530.0 \\
1,054.0 \\
821.0 \\
828.0 \\
1,130.0 \\
873.0 \\
810.0 \\
852.0 \\
1,065.0\end{array}$ & $\begin{array}{l}03 / 74 \\
C 3 / 74 \\
06 / 74 \\
07 / 74 \\
C 8 / 74 \\
08 / 74 \\
09 / 74 \\
09 / 74 \\
09 / 74 \\
10 / 74 \\
11 / 74 \\
12 / 74 \\
01 / 75 \\
03 / 75 \\
10 / 75 \\
12 / 75 \\
04 / 76 \\
04 / 76 \\
05 / 76 \\
08 / 76\end{array}$ & $\begin{array}{l}04 / 74 \\
05 / 74 \\
0 \in / 74 \\
08 / 74 \\
0 \subseteq / 74 \\
C E / 74 \\
09 / 74 \\
1 C / 74 \\
11 / 74 \\
12 / 74 \\
0</ 75 \\
12 / 74 \\
02 / 75 \\
C 4 / 75 \\
11 / 75 \\
1 / 7 \\
04 / 76 \\
05 / 76 \\
C 5 / 76 \\
1 !\end{array}$ & $\begin{array}{r}3,3 \varepsilon 3,265 \\
2,489,342 \\
4,335, \in 25 \\
1,5 \in 7,17 C \\
6,049,644 \\
3, E \varepsilon 7,546 \\
4,755,2 C 8 \\
2,205,091 \\
4,1 \equiv 3,7 \in 5 \\
6,3 C 3,941 \\
4,156,2 \subseteq 8 \\
2,6 \in C, 7 C 1 \\
6,804,954 \\
2,486,535 \\
4,543,17 C \\
2,102,986 \\
1,812,547 \\
58,975 \\
441,53 C \\
1,416,851\end{array}$ & $\begin{array}{r}1 C, E 0 \epsilon, \sum 17 \\
8,023,776 \\
13,526,275 \\
4,55 C, C \in C \\
19,458,506 \\
12,072,74 \epsilon \\
14,45 C, 167 \\
6,908,230 \\
13,77 C, \epsilon \subseteq 5 \\
15,757,729 \\
12,637,857 \\
5,555,455 \\
21,484,038 \\
7,807,5 \epsilon 4 \\
15,155,524 \\
7,540,494 \\
5,872,7 \varepsilon 2 \\
247,602 \\
1,573,248 \\
4,456,4 \varepsilon \epsilon\end{array}$ & $\begin{array}{l}7,216 \\
7,002 \\
5,839 \\
2,837 \\
7,656 \\
5,664 \\
6,258 \\
2,966 \\
7,731 \\
8,400 \\
6,527 \\
6,84 C \\
7,384 \\
5,255 \\
7,281 \\
3,631 \\
2,345 \\
1,63 C \\
1,574 \\
2,284\end{array}$ & $\begin{array}{r}84.9 \\
75.7 \\
7 \Xi .4 \\
1 C C . C \\
8 C .3 \\
59.9 \\
71.2 \\
57.8 \\
88.0 \\
56.1 \\
74.3 \\
78.5 \\
84.1 \\
59.9 \\
C 5.4 \\
54.8 \\
78.8 \\
\text { N/A } \\
\text { N/A } \\
\text { N/A }\end{array}$ & $\begin{array}{l}78.5 \\
78.0 \\
65.4 \\
25.0 \\
77.7 \\
56.6 \\
65.2 \\
30.4 \\
83.1 \\
55.2 \\
71.6 \\
75.8 \\
83.1 \\
56.0 \\
7 E .7 \\
64.0 \\
76.6 \\
\text { N/A } \\
-\mathrm{N} / \mathrm{A} \\
77.5\end{array}$ & $\begin{array}{l}72.0 \\
52.7 \\
6 C .3 \\
16.8 \\
64.7 \\
52.1 \\
61.0 \\
27.5 \\
59.9 \\
64.5 \\
57.6 \\
57.2 \\
73.5 \\
34.5 \\
62.5 \\
21.2 \\
72.1 \\
4 \epsilon .3 \\
. \mathrm{N} / \mathrm{A} \\
5 \mathrm{C} .1\end{array}$ & $\begin{array}{l}74.8 \\
55.0 \\
\epsilon Z .3 \\
1 \epsilon .8 \\
6 \epsilon .5 \\
52.9 \\
62.2 \\
28.8 \\
59.9 \\
85.7 \\
\epsilon 1: 5 \\
58.3 \\
74.2 \\
35.8 \\
6 \epsilon .3 \\
25.3 \\
72.1 \\
4 \epsilon .7 \\
N / A \\
50.1\end{array}$ \\
\hline $\begin{array}{l}\text { BRUNSWICK \#1 } \\
\text { CAL VERT CLIFFS \#2 } \\
\text { SALEM \#I }\end{array}$ & $\begin{array}{r}821.0 \\
845.0 \\
1,090 . \mathrm{C}\end{array}$ & $\begin{array}{l}10 / 76 \\
11 / 76 \\
12 / 76\end{array}$ & $\begin{array}{l}12 / 76 \\
1</ 76\end{array}$ & $\begin{array}{r}30,399 \\
116,011\end{array}$ & $\begin{array}{r}160,372 \\
434, C 58 \\
48,6 \subseteq 7\end{array}$ & $\begin{array}{l}\text { N/A } \\
554 \\
302\end{array}$ & $\begin{array}{l}N / A \\
N / A \\
N / A\end{array}$ & $\begin{array}{l}\text { N/A } \\
\text { N/A } \\
\text { N/A }\end{array}$ & $\begin{array}{l}N / A \\
N / A \\
N / A\end{array}$ & $\begin{array}{l}\text { N/A } \\
\text { N/A } \\
\text { N/A }\end{array}$ \\
\hline
\end{tabular}

1/. Unit capacity factor based on Nuclear Regulatory Commission. (NRC) net design electric rating (DER)

2/ Unit capacity factor based on NRC net maximum dependable capacity (MDC) rating

3/. Not in operation in 1976

4! Put in commercial operation in 1976

NOTE - Cryatal River 3 licensed to operate ln late 1976 with no data available yet 
TABLE 2 - GENERATICN OF THERMAL ENEREY EY CENTRAL STATION NUCLEAR POWER PLANTS (GRCSS TNHH EY YEARS)

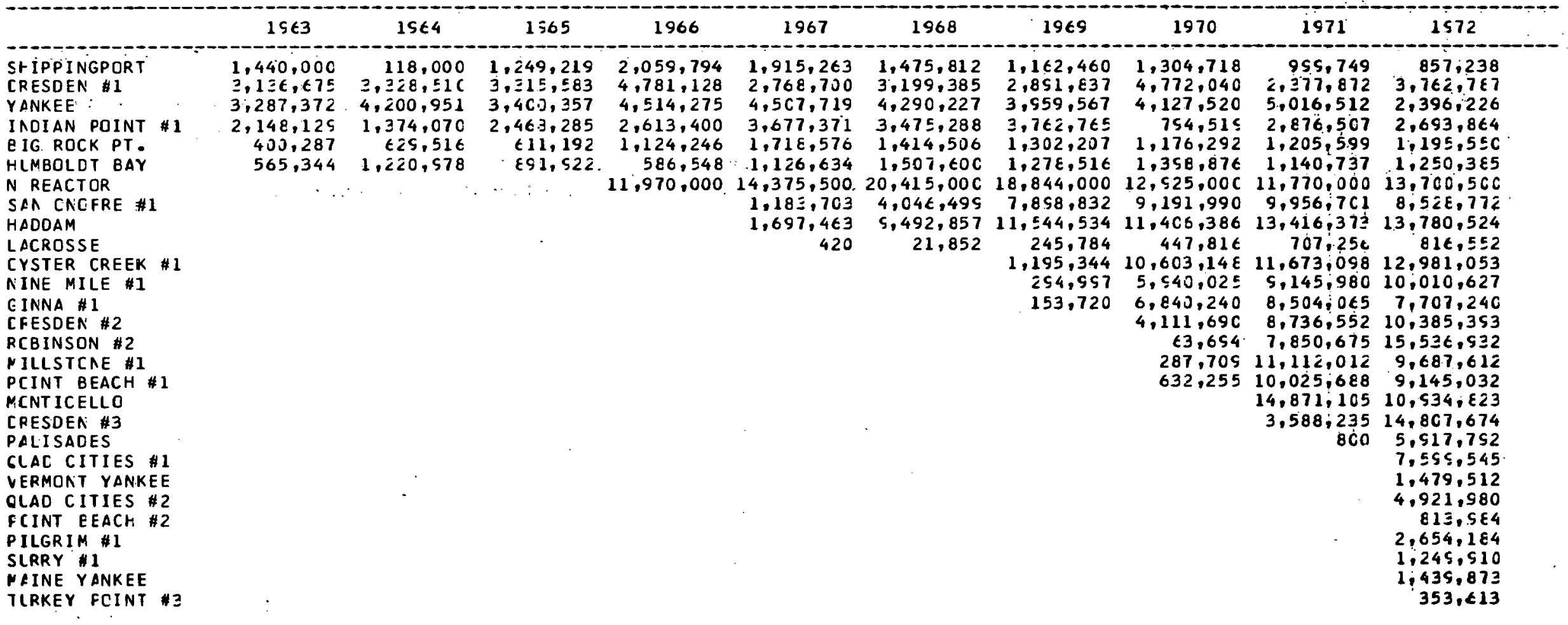

Decommissioned planti:

Hallam: 638,760 TMWH for 1963-1964

E1k River: 1, 272,416 TMWH Еor 1963-1968

Piqua: 256,972 TMH for 1963-1966

CVTR: 859,105 TMNH for 1963-1967

Bonus: 259,588 TMWH for 1964-1967

Pathfinder: 399,241 TMWH for 1966-1967

Peach Bottom 非: 3,722,550 TMWH for 1966-1974

Ferm1 非1: 161,383 TMWH for 1966; 1970-1971 
TABLE 2 - GEMERATICN GF THERNAL ENERGY EY CENTRAL STATION NUCLEAR POWER PLANTS IGRCSS TM.hH EY YEARSI

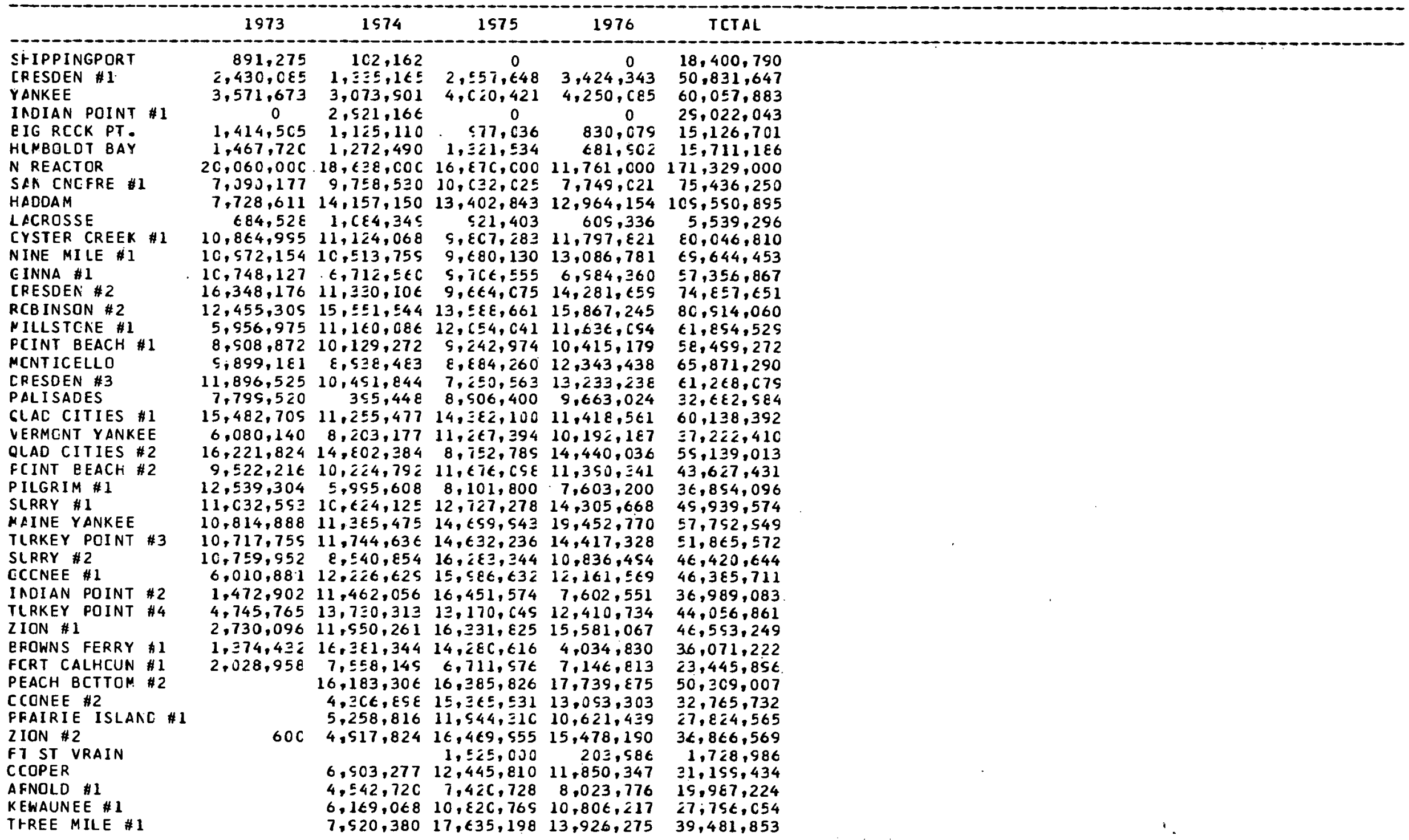


TABLE 2 - GENERATJEN OF THERMAL ENEREY BY CENTRAL STATION NLCLEAR POHER PLANTS IGRCSS TMHH EY YEARSI

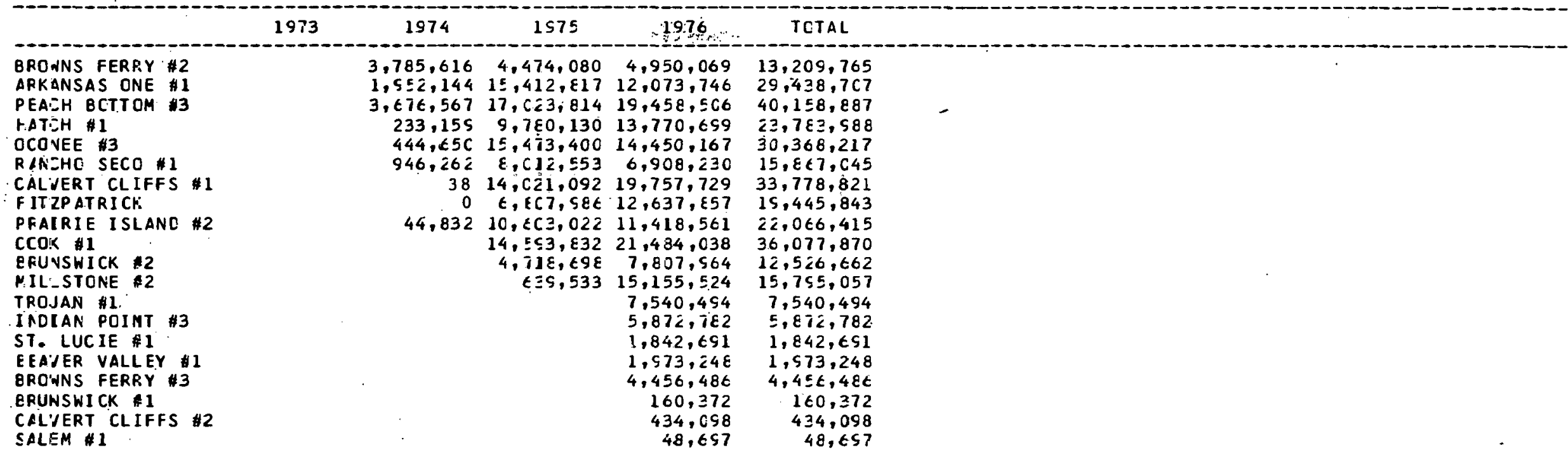


TABLE 3 - GENERATIGA CF ELECTRICITY EY CENTRAL STATION NUCLEAR PCWER FLANTS (GRCSS EMHH EY YEARS)

\begin{tabular}{|c|c|c|c|c|c|c|c|c|c|c|}
\hline & 1963 & 1964 & 1965 & 1966 & 1967 & 1968 & 1969 & 1970 & 1571 & 1572 \\
\hline 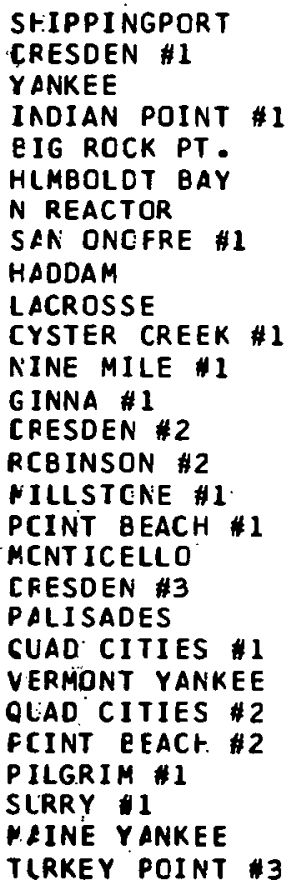 & $\begin{array}{r}395,361 \\
589,721 \\
1,004,088 \\
553,900 \\
130,371 \\
176,025\end{array}$ & $\begin{array}{r}25,406 \\
1,637,511 \\
1,257,556 \\
625,560 \\
203,633 \\
380,222\end{array}$ & $\begin{array}{r}363,067 \\
1, C 18,344 \\
1, C 27,725 \\
1,145,510 \\
150,715 \\
272,064\end{array}$ & $\begin{array}{r}587,270 \\
1,474,832 \\
1,371,620 \\
1,227,785 \\
363,306 \\
1 \epsilon t, 404 \\
1,050,000\end{array}$ & $\begin{array}{r}532,999 \\
853,567 \\
1,348,424 \\
1,752,250 \\
530,110 \\
335,786 \\
2,100,000 \\
386,457 \\
561,512\end{array}$ & $\begin{array}{r}410,470 \\
966,792 \\
1,292,877 \\
1,640,550 \\
448,997 \\
465,510 \\
4,035,000 \\
1,356,055 \\
3,16 \varepsilon, 738 \\
3,245\end{array}$ & $\begin{array}{r}342,487 \\
873,285 \\
1,211,897 \\
1,806,850 \\
421,779 \\
3 \varepsilon \varepsilon, 561 \\
3,780,000 \\
2,751,185 \\
3,841,00 C \\
76,721 \\
351,435 \\
7 C, 567 \\
38,159\end{array}$ & $\begin{array}{r}387,117 \\
1,428,82 \mathrm{C} \\
1,280,213 \\
380,87 \mathrm{C} \\
380,91 \mathrm{C} \\
431,443 \\
2,7 \mathrm{C}, 00 \mathrm{C} \\
3,205,80 \mathrm{C} \\
3,720,14 \mathrm{C} \\
134,270 \\
3,592,855 \\
1,517,70 \mathrm{E} \\
2,314,072 \\
1,252,204 \\
17,42 \mathrm{C} \\
67,251 \\
213,27 \mathrm{C}\end{array}$ & $\begin{array}{r}258,568 \\
645, t \in \varepsilon \\
1,520,005 \\
1,345,360 \\
388,6 \geq 5 \\
346,810 \\
2,602,000 \\
3,468,000 \\
4,399,442 \\
210,460 \\
3,972,659 \\
3,632,972 \\
2,871,730 \\
2,806,520 \\
2,565,345 \\
3,747,156 \\
3,446,080 \\
1,465,724 \\
1,188,630 \\
26 C\end{array}$ & 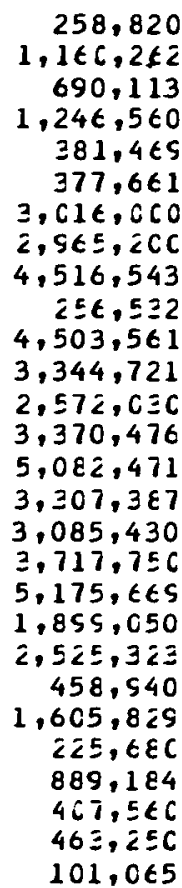 \\
\hline
\end{tabular}

Decomm1sstoned plants:

Hallam: 192,458 EMWH for 1963-1964

Eik River: 510,598 EMWH for 1963-1968

P1qua: 70,601 EMWH for 1963-1966

CVTR: 212,216 EMWH for 1963-1967

Bonus : 68,297 EMWH for 1964-1967

Pathfinder: 96,450 EMWH for 1966-1967

Peach Bottoo \$1: 1,385,919 ENWH for $1967-1974$

rermi: 33,430 EMWH for $1968 ; 1970-1971$ 
TABLE 3 - GEAERATICA CF ELECTRICITY EY CENTRAL STATIGN NUCLEAR POHER PLANTS (GRCSS EMWH EY YEARS)

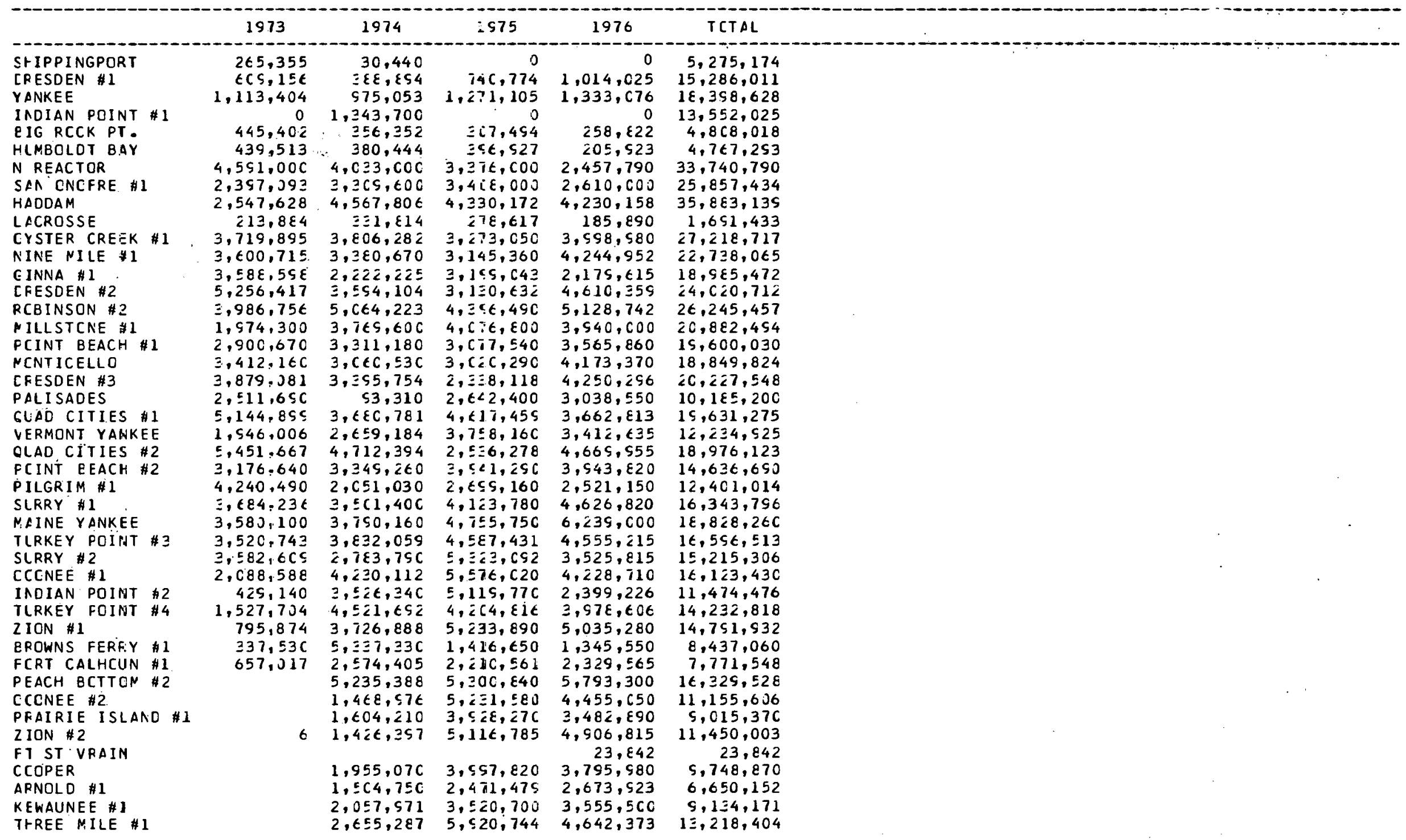


TABLE 3 - GENERATICA CF ELECTFICITY EY CENTRAL STATICN NUCLEAR PCWER PLANTS (GAOSS EMWH EY YEARS)

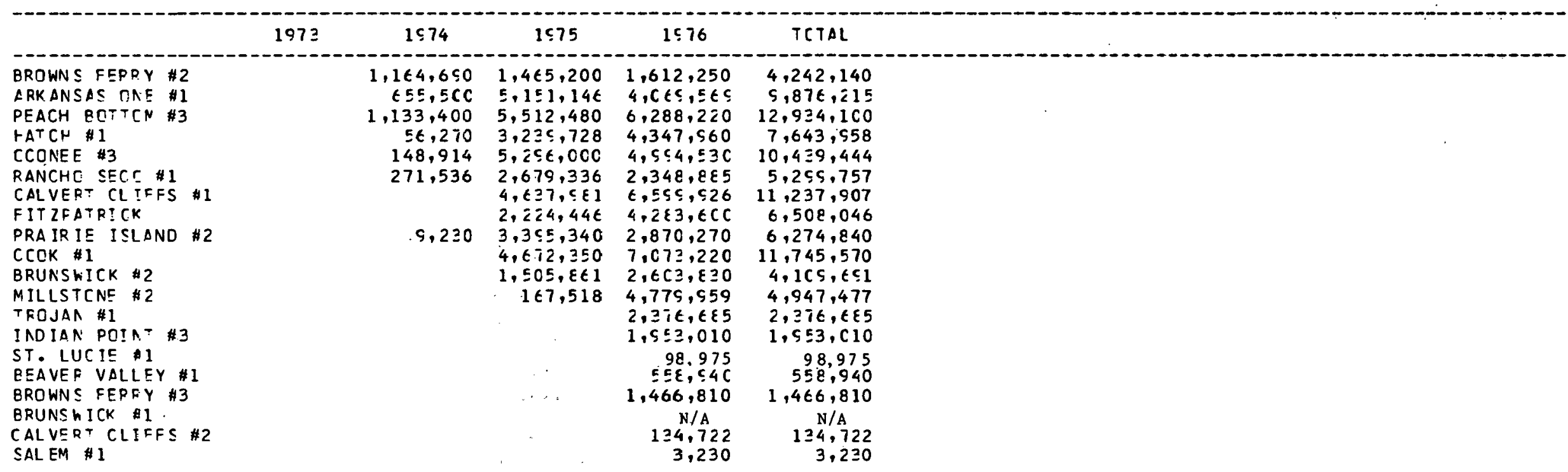


TABLE 4 - GEAER/TICA. OF ELECTRICITY OY CENTRAL STATION NUCLEAR POLER FLANTS (NET EMHE. EY YEARS)

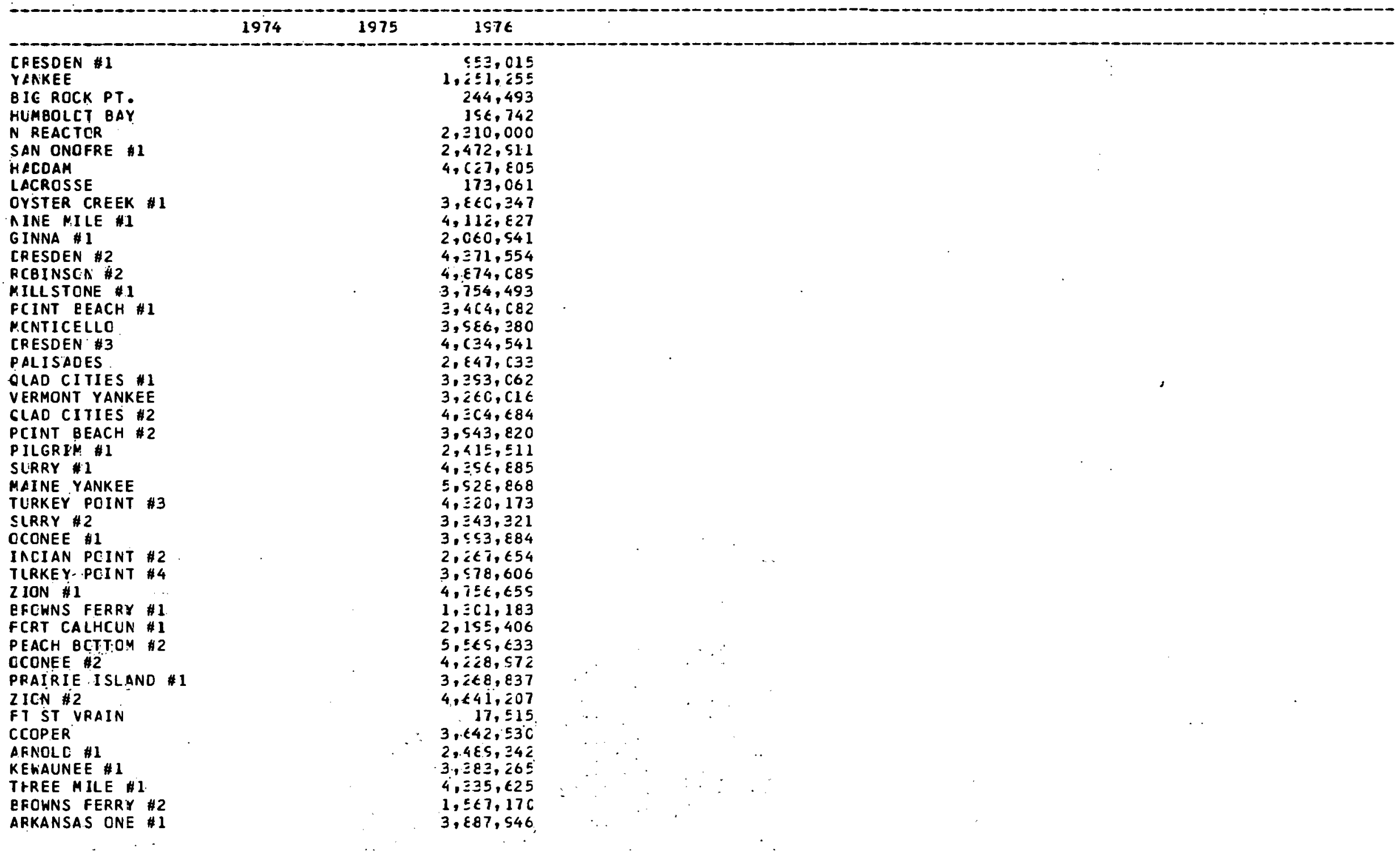


TABLE 4 - EENERATIOA OF ELECTRICITY EY CENTRAL STATICN NUCLEAR PCHER FLANTS (NET ENHT. EY YEARS)

\begin{tabular}{|c|c|}
\hline 1574 & 1975 \\
\hline $\begin{array}{l}\text { PEACH BOTTOM \#3 } \\
\text { HATCH \#1 } \\
\text { CCONEE \#3 } \\
\text { RANCHC SECC \#1 } \\
\text { CALVERT CLIFFS \#1 } \\
\text { FITZPATRICK } \\
\text { PRAIRIE ISLANC \#2 } \\
\text { CCOK \#1 } \\
\text { EFUNSWICK \#2 } \\
\text { HILLSTONE \#2 } \\
\text { TROJAN \#1 } \\
\text { INDIAN POINT \#3 } \\
\text { ST. LUCIE \#1 } \\
\text { EEAVER VALLEY \#1 } \\
\text { BRGWNS FERRY \#3 } \\
\text { BRUNSWICK \#1 } \\
\text { CALVERT CLIFFS \#2. }\end{array}$ & $\begin{array}{r}6, C 49,644 \\
4,133,765 \\
4,755,208 \\
2,2 C 5, C 91 \\
6,3 C 3,541 \\
4,256,258 \\
3, \equiv 53, C 62 \\
6,8 C 4,554 \\
2,486,535 \\
4,543,170 \\
2,162,586 \\
1,872,547 \\
473,155 \\
441,530 \\
1,416,891 \\
30,399 \\
116, C 11\end{array}$ \\
\hline
\end{tabular}


TABLE 5 - CENTRAL STATION NUCLEAR PCWER REACTCR AVAILABILITY - LNITS OPERABLE IN IST6 (HOURS REACTCR CRITICAL FER YEAR PLUS RESERVE SHUTCChN HOURS)

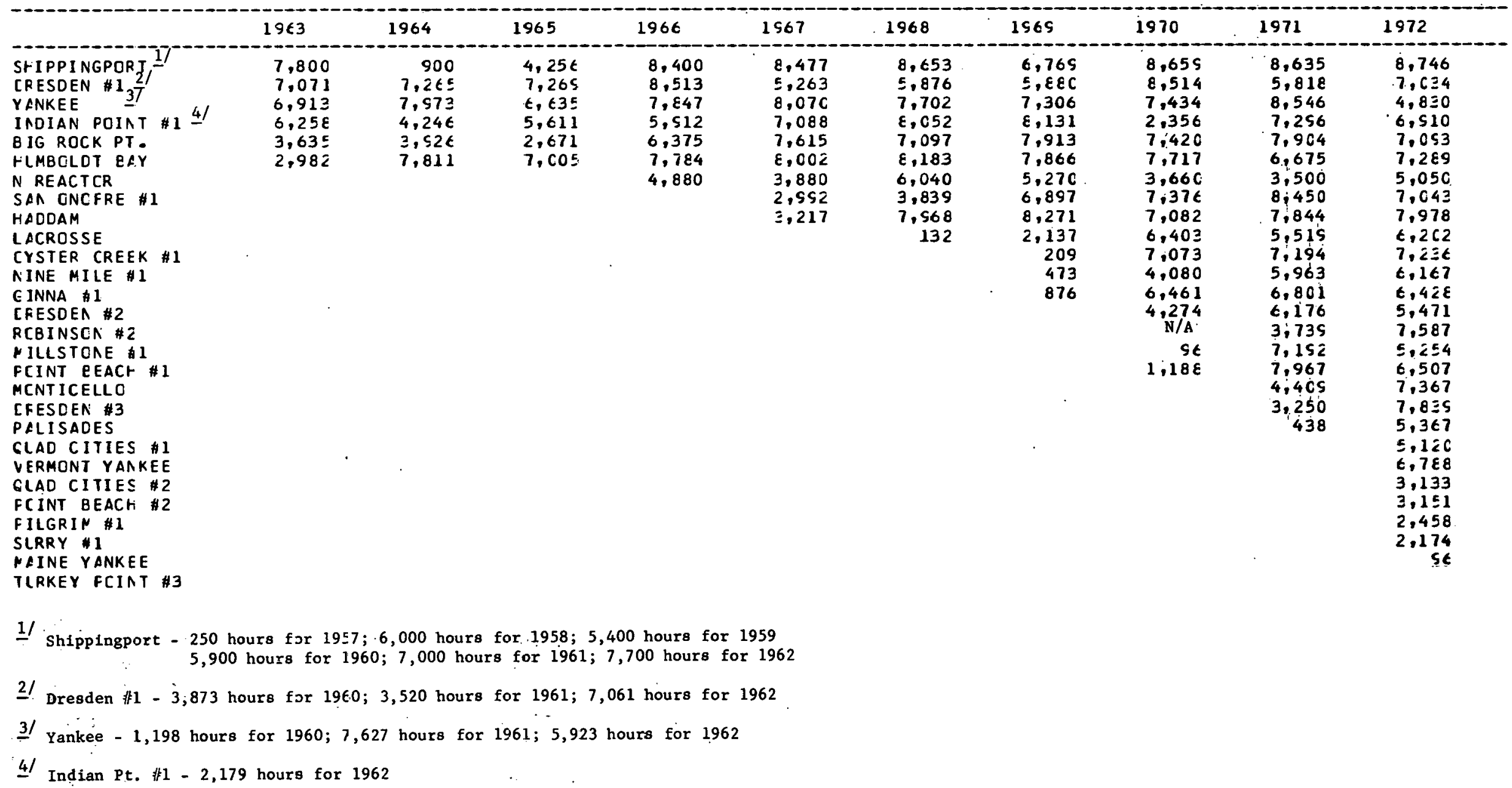


TABLE 5 - CENTFAL STATICN NUCLEAR POWER REACTOR AVAILABILITY - UNITS CFERAELE IA IC76 (HCURS REACTCA CFITICAL FEF YEAR PLUS RESERVE SHUTCOWN HOURS)

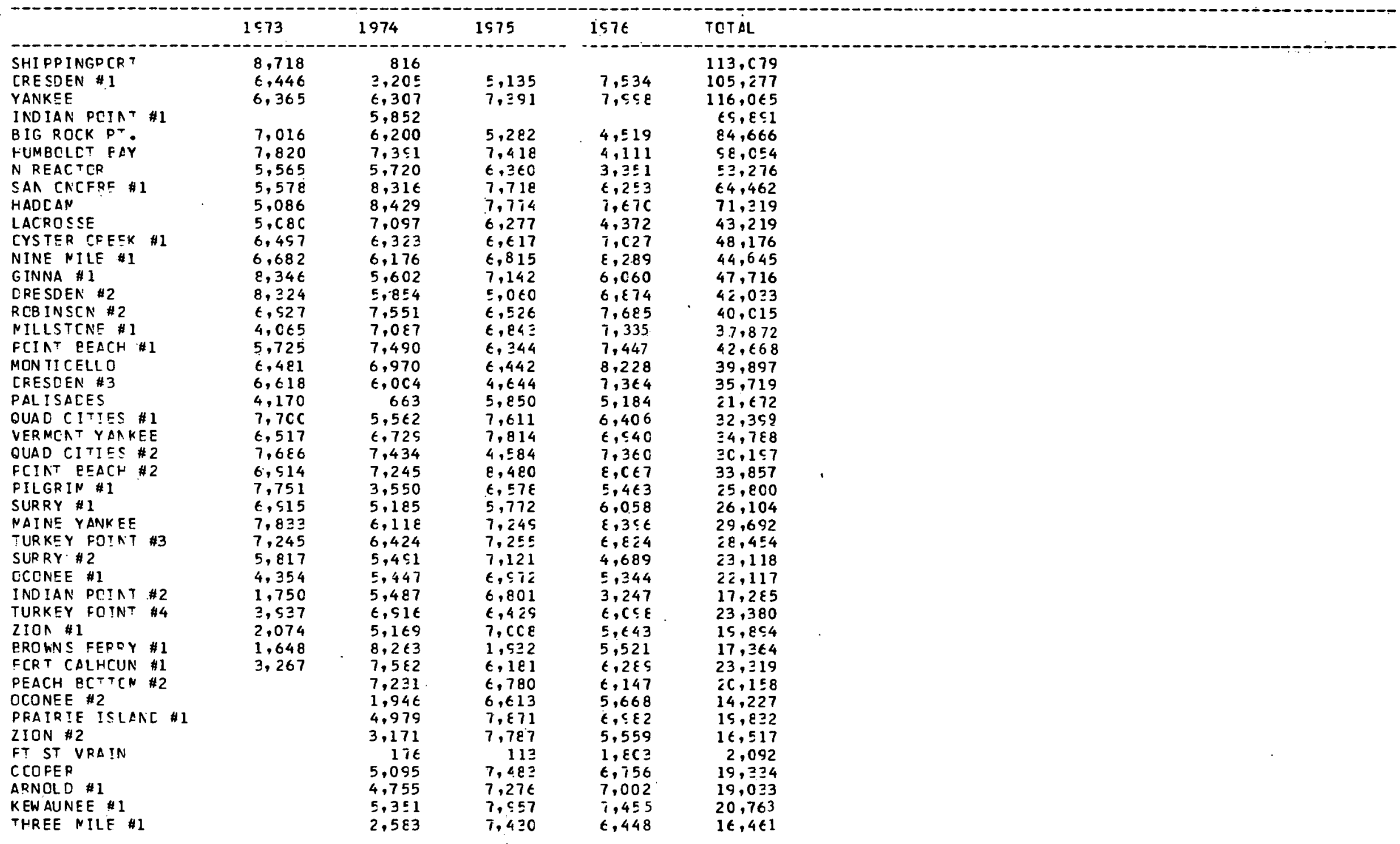


TABLE 5 - CENTFAL STATICN NUCLEAR FOWER REACTOR AVAILABILITY - UNITS CFEFAELE IN IS76 (HCUFS REACTOR CRITICAL FEF YEAR PLUS RESEFVE SHUTCOWN HOLRSI

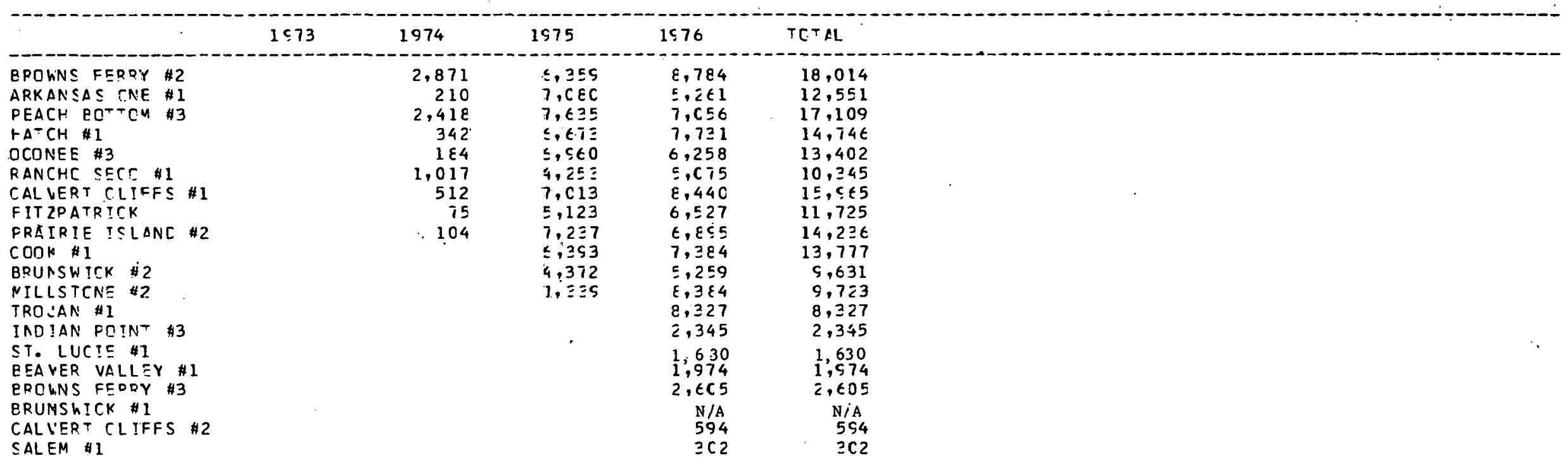


TAELE 6 - FCWEF REACTOR AVAILABILITY FACTCRS (W) - UNITS CPERAELE IN ISTE

\begin{tabular}{|c|c|c|c|c|c|c|c|c|c|c|c|c|}
\hline & 1961 & $19 \in 2$ & 1963 & 1964 & 1SE5 & $1 S E t$ & 1967 & $1 s \in \varepsilon$ & $1969^{\circ}$ & 1970 & $197 i$ & 1972 \\
\hline $\begin{array}{l}\text { SHIPPINGPCRT } \frac{1}{2} \\
\text { CRESOEN \#1 } \\
\text { YANKEE } \\
\text { INOIAN PCINT \#1 } \\
\text { BIG ROCK PT. } \\
\text { HLMBOLOT BAY } \\
\text { N REACTCR } \\
\text { SAN ONOFRE \#1 } \\
\text { HACCAM } \\
\text { LACROSSE } \\
\text { CYSTER CREEK \#1 } \\
\text { A INE NILE \#I } \\
\text { GINNA \#1 } \\
\text { CFESDEN \#2 } \\
\text { RCBINSCN \#2 } \\
\text { NILLSTCNE \#1 } \\
\text { FCINT EEACH \#1 } \\
\text { NCNTICELLC } \\
\text { CRESDEN \#3 } \\
\text { FALISACES } \\
\text { QLAD CITIES \#1 } \\
\text { VERMONT YANKEE } \\
\text { GLAD CITIES \#2 } \\
\text { PCINT BEACH \#2 } \\
\text { PILGRIM \#I } \\
\text { SLRRY \#I } \\
\text { MAINE YANKEE } \\
\text { TLRKEY POINT \#3 }\end{array}$ & $\begin{array}{l}79.93 \\
40.20 \\
87.00\end{array}$ & $\begin{array}{l}87.50 \\
8 C .60 \\
67.60 \\
60.50\end{array}$ & 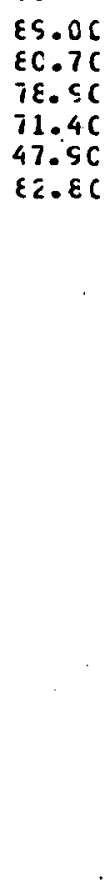 & $\begin{array}{l}13.25 \\
82.70 \\
91.10 \\
48.40 \\
42.80 \\
85.10\end{array}$ & $\begin{array}{l}48.5 \varepsilon \\
83.0 C \\
75.7 C \\
64.0 C \\
30.30 \\
75.7 C\end{array}$ & $\begin{array}{l}55.70 \\
57.20 \\
85.58 \\
67.50 \\
75.00 \\
8 E .80 \\
77 . C C\end{array}$ & $\begin{array}{l}56.6 C \\
60.10 \\
92.12 \\
80.5 C \\
89.7 C \\
51.30 \\
44.30 \\
62.50 \\
87.60\end{array}$ & $\begin{array}{l}98.50 \\
67.16 \\
87.68 \\
91.7 C \\
8 C .5 C \\
93.20 \\
65.00 \\
43.7 C \\
90.7 C \\
1.51\end{array}$ & $\begin{array}{r}77.20 \\
67.10 \\
83.40 \\
92.80 \\
51.50 \\
89.80 \\
60.00 \\
78.7 C \\
94.40 \\
24.39 \\
96.63 \\
5.4 C \\
65.3 C\end{array}$ & $\begin{array}{r}98.80 \\
97.20 \\
84.86 \\
26.90 \\
86.4 \mathrm{C} \\
88.1 \mathrm{C} \\
42.00 \\
84.2 \mathrm{C} \\
80.84 \\
73.05 \\
80.75 \\
61.10 \\
72.5 \mathrm{C} \\
68.00 \\
\mathrm{~N} / \mathrm{A} \\
100 . \mathrm{CC} \\
89.00\end{array}$ & $\begin{array}{l}98.60 \\
66.40 \\
97.56 \\
83.30 \\
90.04 \\
76.20 \\
40.00 \\
56.46 \\
89.54 \\
62.99 \\
82.12 \\
70.33 \\
77.63 \\
70.50 \\
51.93 \\
82.00 \\
93.59 \\
62.50 \\
83.10\end{array}$ & $\begin{array}{l}99.60 \\
79.50 \\
55.15 \\
78.70 \\
81.80 \\
83.00 \\
58.00 \\
80.18 \\
90.81 \\
70.60 \\
82.40 \\
72.60 \\
71.95 \\
64.60 \\
88.65 \\
60.00 \\
74.50 \\
83.90 \\
52.70 \\
61.10 \\
79.30 \\
100.00 \\
65.28 \\
87.98 \\
55.50 \\
49.20 \\
1.05 \\
49.00\end{array}$ \\
\hline
\end{tabular}

\footnotetext{
1/ Shippingport: 67.3 in 1960

2/ Dresden \#1: 58.7 in 1960
} 
TABLE 6 - FChEF reactCR AVAILAEILITY FACTORS (q) - UNITS OfERABLE IN ICTE

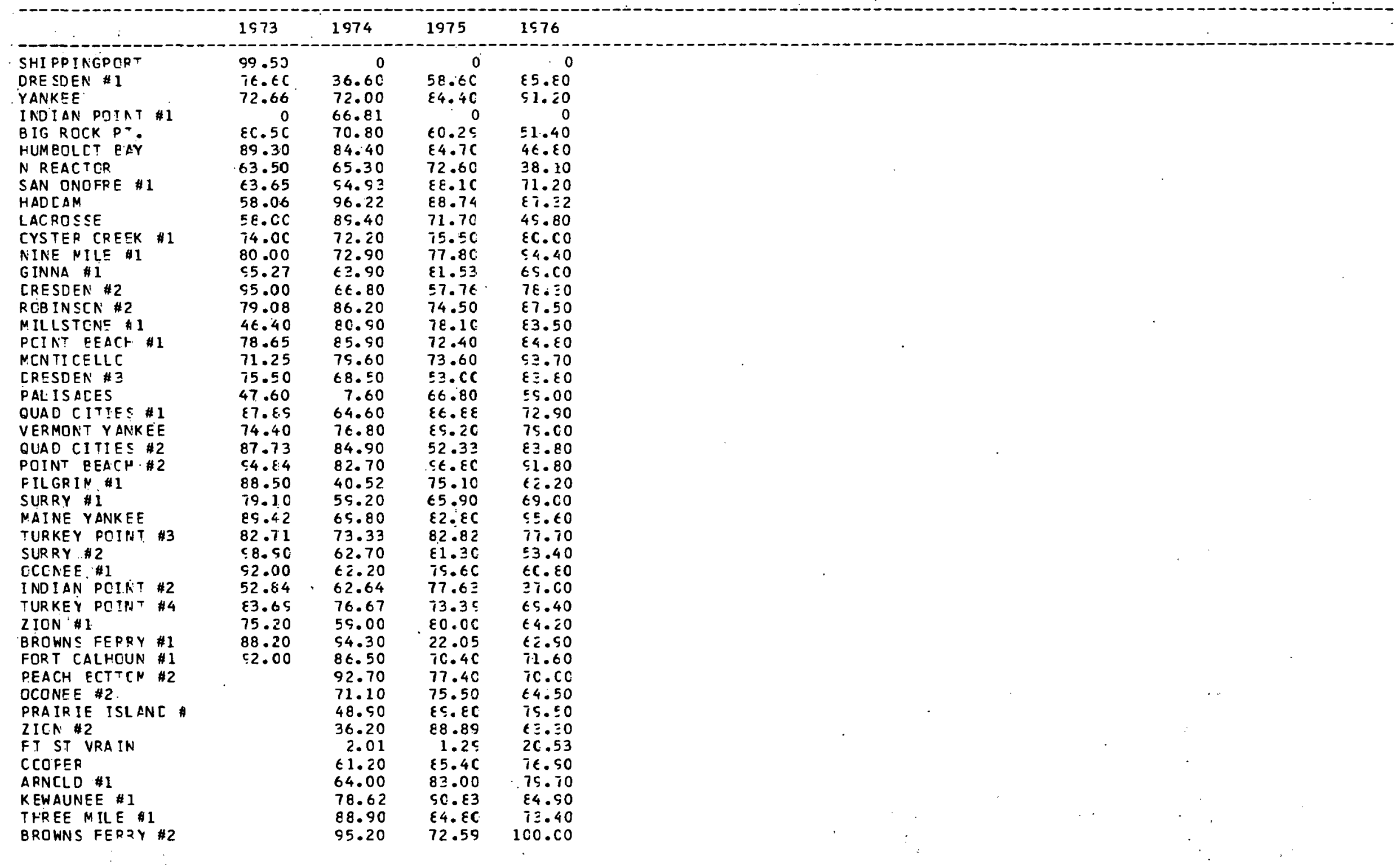


TAELE 6 - POWER REACTCR AVAILAEILITY. FACTORS (q) - UNITS CFEFAELE IN 1976

\begin{tabular}{|c|c|c|c|c|}
\hline 1973 & 1974 & 1975 & $1 \subseteq 76$ & \\
\hline $\begin{array}{l}\text { ARKANSAS CNE \#1 } \\
\text { FEACH ECTTCN \#3 } \\
\text { HATCH \#1 } \\
\text { CCONEE \#3 } \\
\text { RANCHC SECC \#1 } \\
\text { CAL VERT CLIFFS \#1 } \\
\text { FITZPARICK } \\
\text { PRAIRIE ISLAND \#2 } \\
\text { COOK \#I } \\
\text { ERUNSWICK \#2 } \\
\text { MILLSTCNE \#2 } \\
\text { TRCJAN \#1 } \\
\text { INDIAN PCINT \#3 } \\
\text { ST LUCIE \#1 } \\
\text { EEAVER VALLEY \#1 } \\
\text { BROWNS FERRY \#3 } \\
\text { BRUNSWICK \#I } \\
\text { CAL VERT CLIFFS \#2 } \\
\text { SALEM I }\end{array}$ & $\begin{array}{r}68.30 \\
100.00 \\
3.90 \\
47.50\end{array}$ & $\begin{array}{l}80.8 C \\
\varepsilon 7.16 \\
i \epsilon .18 \\
7 C .5 C \\
48.60 \\
50.4 C \\
i 4 . C C \\
57.30 \\
86.2 C \\
55.49 \\
79.90\end{array}$ & $\begin{array}{l}59.50 \\
\varepsilon C . .0 \\
88.00 \\
i 1.20 \\
57.80 \\
\subseteq E .10 \\
i 4 . \equiv 0 \\
7 \varepsilon .50 \\
\varepsilon 4.10 \\
5 \subseteq . \varepsilon 7 \\
\text { S..40 } \\
\subseteq 4 . \varepsilon 0 \\
i \varepsilon . E 0 \\
\text { N/A } \\
\text { N/A } \\
\text { N/A } \\
\text { N/A } \\
\text { N/A } \\
\text { N/A }\end{array}$ & $\cdot$ \\
\hline
\end{tabular}




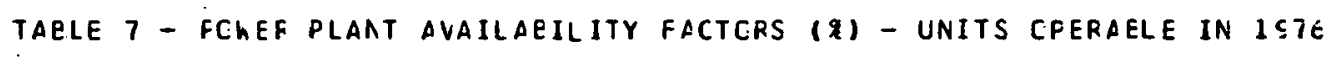

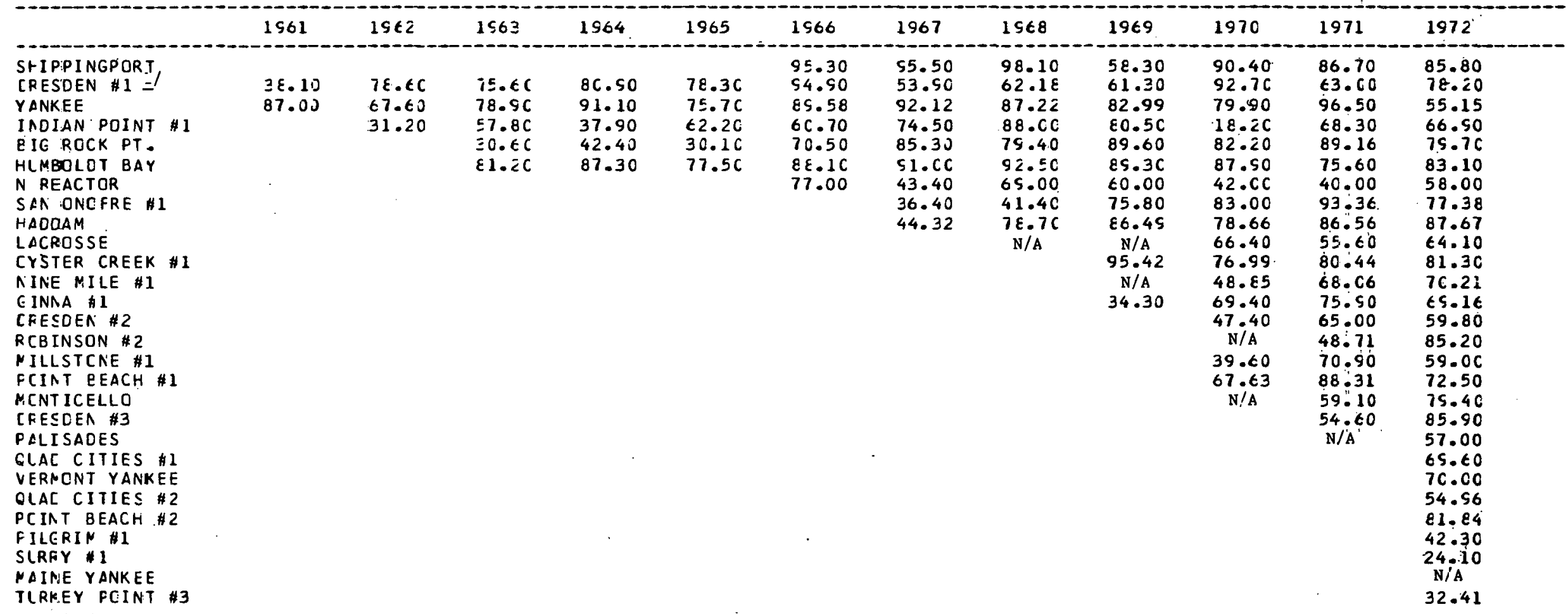


TAELE 7 - FOWEF PLANT AVAILABILITY FACTORS (\%) - UNITS OPERAELE IN 1 \$ 76

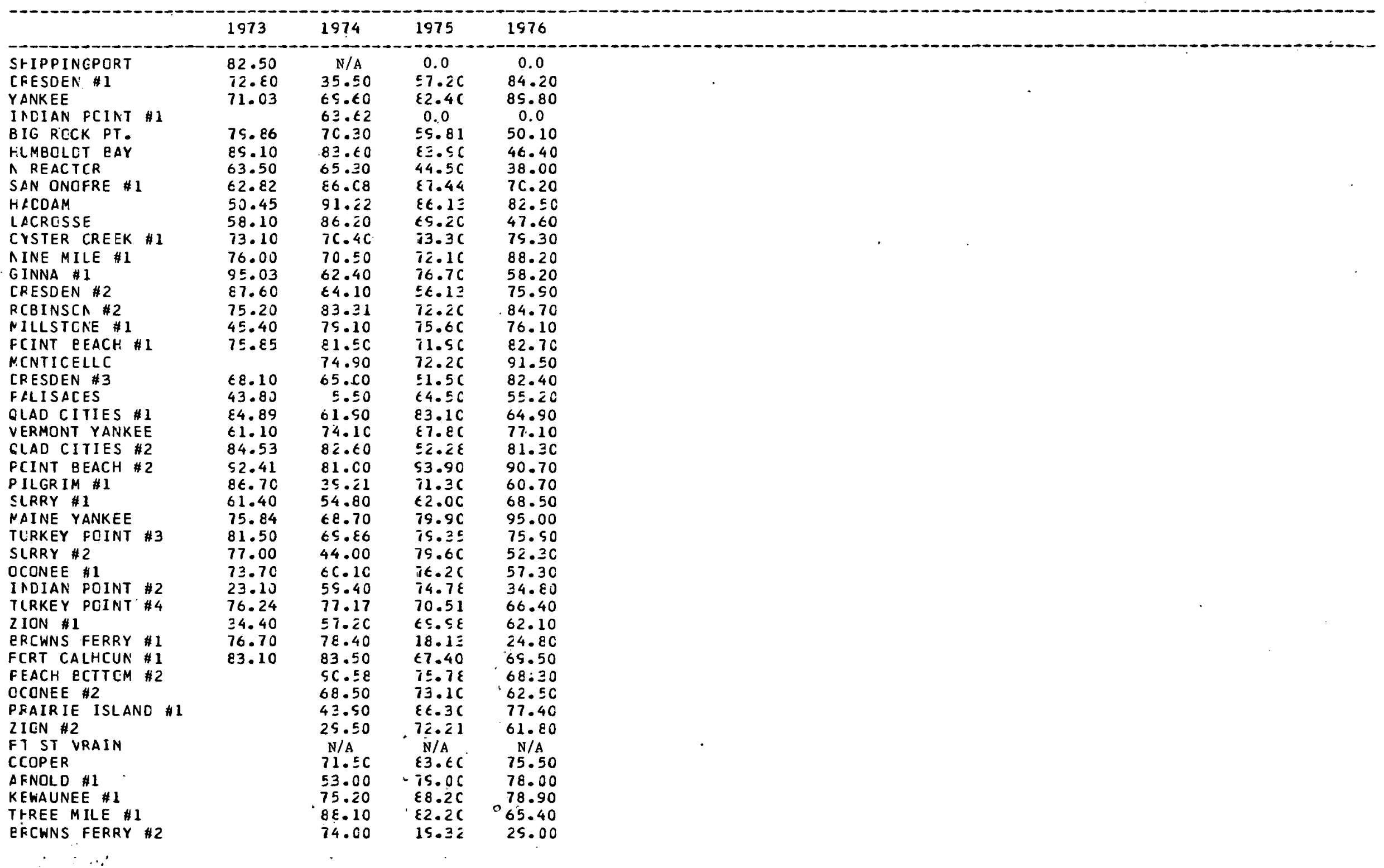




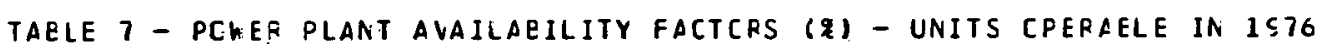

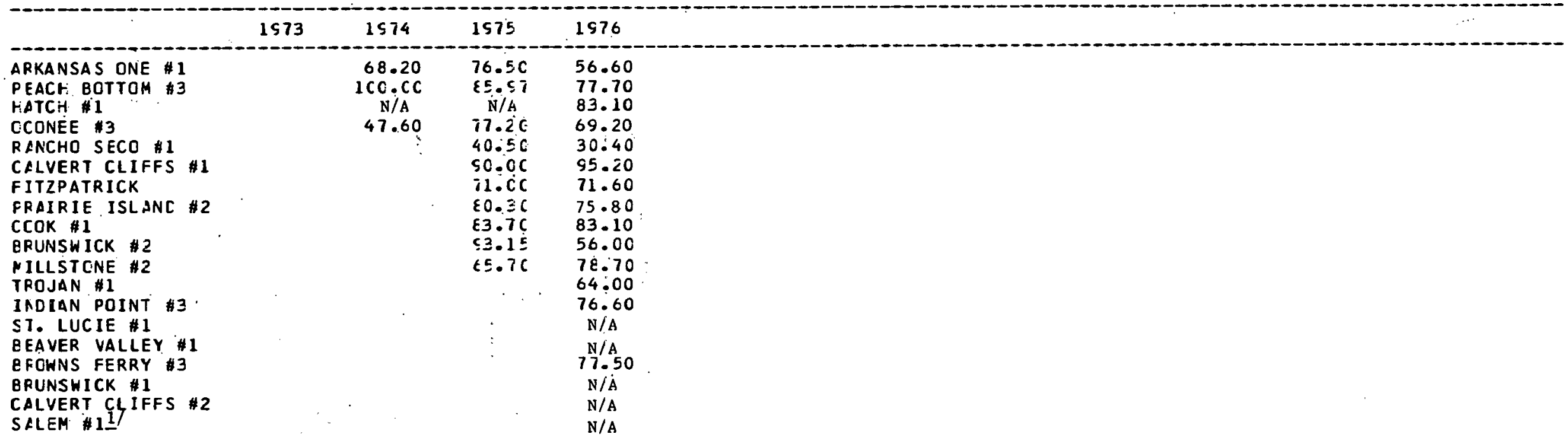

1/ From date of commercial operation 
TABLE 8 - FCWER PLANT CAPACITY FACTCRS (\$) - UNITS OPERABLE IN 1 STE

\begin{tabular}{|c|c|c|c|c|c|c|c|c|c|c|c|c|}
\hline & 1961 & 1962 & 1963 & 1964 & 1965 & $19 t E$ & 1967 & $1 S \in 8$ & 1569 & 1970 & 1971 & 1972 \\
\hline 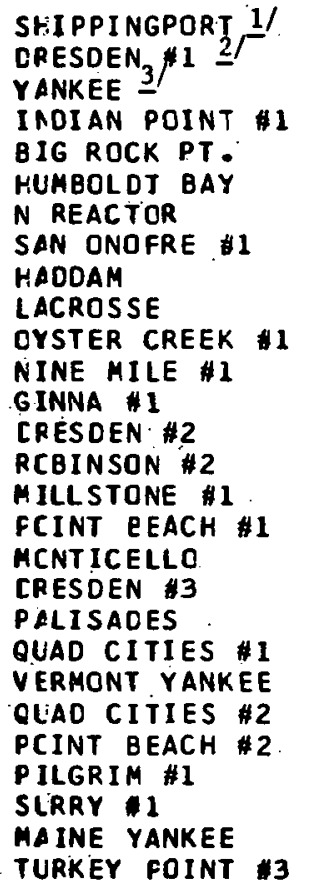 & $\begin{array}{l}59.00 \\
33.00 \\
76.00\end{array}$ & $\begin{array}{l}62.00 \\
73.00 \\
55.0 C \\
27.70\end{array}$ & $\begin{array}{l}66.0 \mathrm{C} \\
53.8 \mathrm{C} \\
65.0 \mathrm{C} \\
38.0 \mathrm{C} \\
35.3 \mathrm{C} \\
77.8 \mathrm{C}\end{array}$ & $\begin{array}{l}4.00 \\
56.20 \\
79.80 \\
24.60 \\
42.20 \\
83.30\end{array}$ & $\begin{array}{l}42.0 C \\
55.4 C \\
64.95 \\
4 E .4 C \\
25.03 \\
55.50\end{array}$ & $\begin{array}{l}67.00 \\
80.20 \\
85.89 \\
56.30 \\
55.30 \\
36.50 \\
41.00\end{array}$ & $\begin{array}{l}60.80 \\
46.40 \\
85.70 \\
68.30 \\
80.70 \\
74.60 \\
42.00 \\
21.30 \\
31.14\end{array}$ & $\begin{array}{c}46.8 \mathrm{C} \\
52.41 \\
81.48 \\
64 . C \mathrm{C} \\
68.20 \\
81.5 \mathrm{C} \\
57 . \mathrm{CC} \\
33.6 \mathrm{C} \\
73.37 \\
\text { N/A }\end{array}$ & $\begin{array}{c}39.10 \\
47.5 \mathrm{C} \\
75.30 \\
72.1 \mathrm{C} \\
64.20 \\
68.20 \\
56.0 \mathrm{C} \\
69.20 \\
74.97 \\
\mathrm{~N} / \mathrm{A} \\
92.67 \\
15.64\end{array}$ & $\begin{array}{l}49.10 \\
77.7 C \\
78.75 \\
14.010 \\
57.58 \\
75.80 \\
38.0 C \\
80.57 \\
71.34 \\
42.6 C \\
74.25 \\
42.40 \\
57.77 \\
23.30 \\
25.5 C \\
30.00\end{array}$ & $\begin{array}{l}34.10 \\
35.30 \\
95.44 \\
60.40 \\
59.15 \\
60.90 \\
40.00 \\
87.53 \\
83.92 \\
43.70 \\
77.54 \\
63.36 \\
65.60 \\
37.70 \\
40.73 \\
63.20 \\
76.30 \\
49.20 \\
35.70\end{array}$ & $\begin{array}{l}29.5 \mathrm{C} \\
62.50 \\
42.50 \\
48.40 \\
57.5 \mathrm{C} \\
6 t .10 \\
40.00 \\
74.52 \\
85.56 \\
54.6 \mathrm{C} \\
75.58 \\
60.50 \\
64.05 \\
47.4 \mathrm{C} \\
80.40 \\
54.5 \mathrm{C} \\
65.41 \\
74.51 \\
72.8 \mathrm{C} \\
57.00 \\
64.01 \\
37.50 \\
39.35 \\
14.77 \\
25.3 \mathrm{C} \\
47.30 \\
\mathrm{~N} / \mathrm{A} \\
10 . \mathrm{C} \Xi\end{array}$ \\
\hline
\end{tabular}

1/ Shippingport: 32.0 in 1959; 45.0 in 1960

2/ Dresden f1: 21.8 in 1960

3/ Yankee: 25.0 in 1960 
TABLE \& - PCHER PLANT CAPACITY FACTCRS (Z) - UAITS GPEFAELE IA $197 \dot{2}$

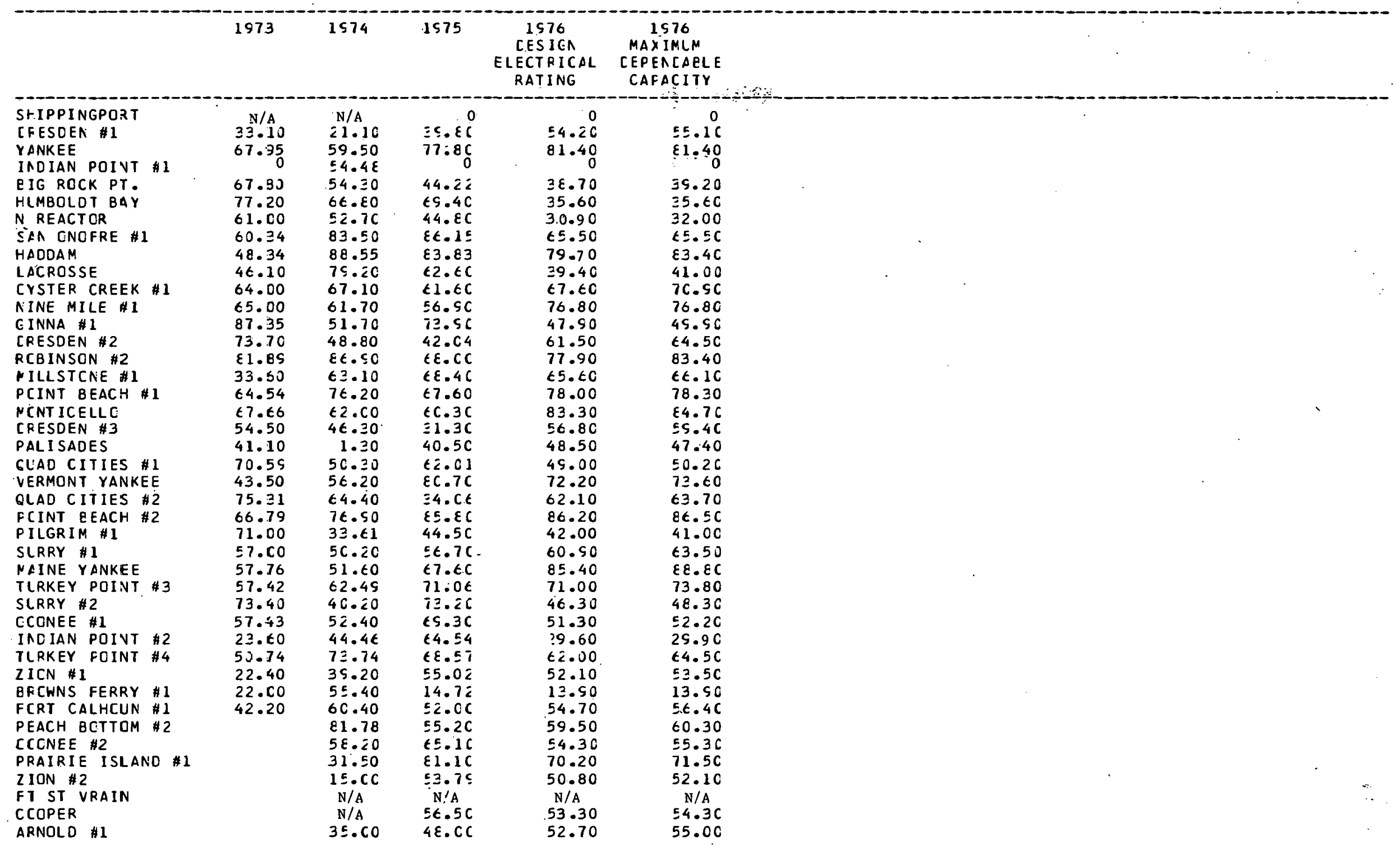


TABLE 8 - FCHER PLANT CAPACITY FACTORS (\%) - UNITS OPERABLE IN 1976

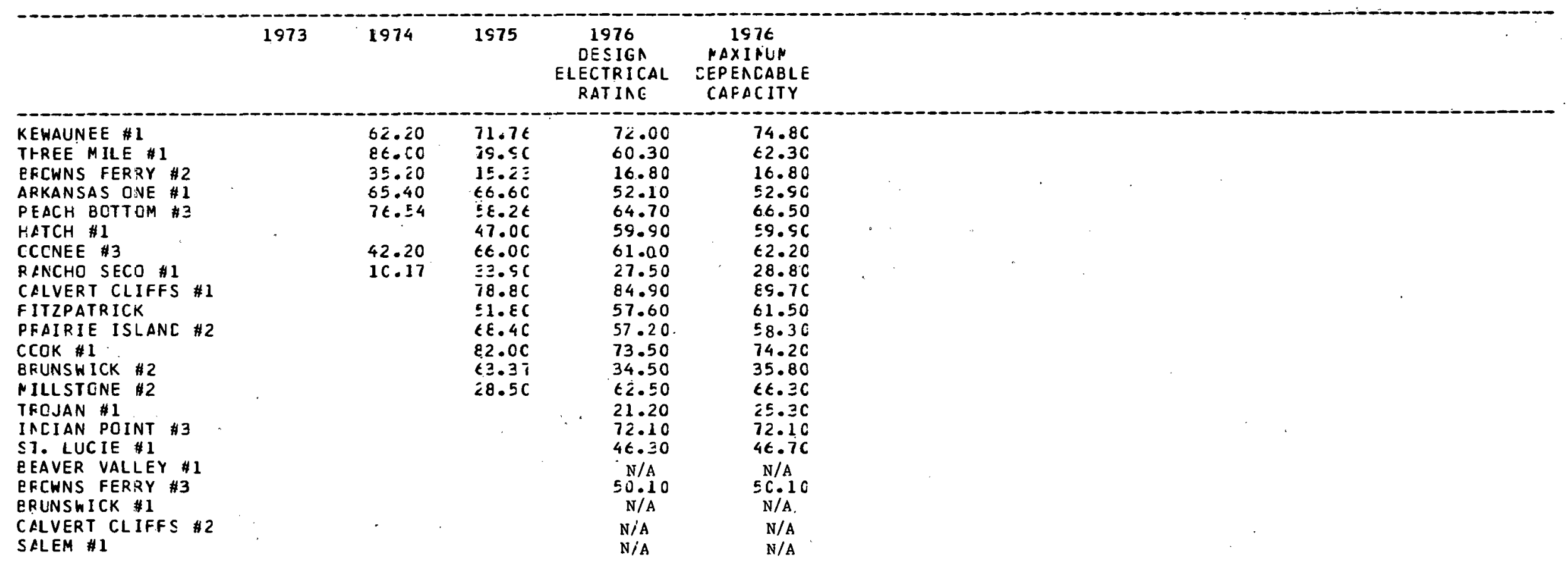




\section{HISTORY OF MAJOR SHUTDOWNS - OPERABLE PLANTS \\ CENTRAL STATION POWER}

Noㅡㅁㅡ:

Only shutdowns of five days or more are listed in this section. These shutdown periods include time for refueling as well as maintenance and repair.

The legend for the headings in the following pages is as follows:

MWt

$M W e$ (net)

CRIT-L

ELECTRIC

DOWNTIME

UPTIME
- Megawatts thermal rating of nuclear unit

- Megawatts electrical (net) rating of nuclear unit

- Month and year in which unit achieved initial criticality

- Month and year in which unit initially delivered electricity to the utility system

- Date on which unit was shut down

- Date on which unit was started up again 


\begin{tabular}{|c|c|c|c|c|c|c|c|}
\hline MHT & MWEE-NET & $C R I T-L$ & ELECTRIC & CCWN-A & {$[K N-C A] E$} & LF-DATE & REASCA \\
\hline $2 \div 8.0$ & 850.0 & $\mathrm{CB} / 74$ & 08,74 & $\begin{array}{r}1 \\
2 \\
3 \\
4 \\
5 \\
6 \\
7 \\
8 \\
9 \\
10\end{array}$ & $\begin{array}{l}C E / 22 / 74 \\
C \subseteq / 12 / 74 \\
1: 11 / 74 \\
1 C / 25 / 74 \\
C \geqq 1 / 1 S / 75 \\
1] / 24 / 75 \\
1 \geq / 21 / 75 \\
C \equiv / 1 S / 76 \\
00 / 21 / 76 \\
C E / 07 / 76\end{array}$ & $\begin{array}{l}C S / 01 / 74 \\
C S / 22 / 74 \\
10 / 22 / 74 \\
11 / 18 / 74 \\
C 5 / 07 / 75 \\
11 / 07 / 75 \\
01 / 17 / 76 \\
C 6 / 1 C / 76 \\
06 / 29 / 76 \\
C S / 13 / 76\end{array}$ & $\begin{array}{l}\text { ACCEC HANGEFS FCR SUPPCRT OF PRESSURIZER RELIEF LINE } \\
\text { GENEFATCR.EXCITER BEARINE FAILURE } \\
\text { LEAK IN DRAIN PIPING ON REACTOR COCLANT SYS } \\
\text { LEAK IN DRAIN PIFING ON REACTOR COOLANT SYS } \\
\text { INSTALLATICA OF STEAH LINE EREAK } \\
\text { CCNTRG ROD INTERCHANGE \& CTHER MAINTENANCE } \\
\text { RATCHET JRIF RESULTING IN CANAGEC CRCM } \\
\text { FAILLRE CF REACTCR SFECINEA TLBE HCLEEFS } \\
\text { REPLACE BEARINGS \& REPAIR STATCR } \\
\text { REPLACE REACTCR CCOLANT FUNF STAFT SEALS }\end{array}$ \\
\hline
\end{tabular}


DLANE ARNOLD ENERGY CENTER UNIT 1

\begin{tabular}{|c|c|c|c|c|c|c|c|}
\hline MWT & MWE-NET & CRIT-L & ELECTRIC & COWN-\# & ChN-CATE & UP-OATE & REASCN \\
\hline 1593.0 & 538.0 & $03 / 7.4$ & $05 / 74$ & $\begin{array}{l}1 \\
2 \\
3 \\
4 \\
5 \\
6 \\
7 \\
8\end{array}$ & $\begin{array}{l}C 3 / 25 / 74 \\
C E / 14 / 74 \\
C S / 11 / 74 \\
C 2 / C 8 / 75 \\
C 4 / 19 / 75 \\
C 6 / 06 / 75 \\
C 2 / 14 / 76 \\
C 5 / 1 S / 76\end{array}$ & $\begin{array}{l}04 / 26 / 74 \\
08 / 19 / 74 \\
09 / 24 / 74 \\
02 / 17 / 75 \\
04 / 24 / 75 \\
07 / 19 / 75 \\
04 / 14 / 76 \\
05 / 26 / 76\end{array}$ & $\begin{array}{l}\text { STARTUP TESTING } \\
\text { STARTLF TESTING } \\
\text { REPAIR OF RELIEF VALVES } \\
\text { INSPECTICN CF CRYWELL PIPINE } \\
\text { REPAIR \& TESTING CF HPCI ShING CHECKS \& STCF CHK VALVES } \\
\text { VIBRATION OF IN-CORE INSTRUMENTATION TLBES } \\
\text { REFLELING } \\
\text { MAINTENANCE CUTAGE }\end{array}$ \\
\hline
\end{tabular}


REAVER VALLEY FOHER STATIEN UNIT 1

\begin{tabular}{|c|c|c|c|c|c|c|c|}
\hline NhT & NWE-NET & CRIT-L & ELECTRIC & $C C h N-6$ & CWN-CATE & UP-DATE & REASCN \\
\hline 2660.0 & 852.0 & $05 / 76$ & $05 / 76$ & $\begin{array}{l}1 \\
2 \\
3 \\
4 \\
5 \\
6\end{array}$ & $\begin{array}{l}C 1 / 3 C / 76 \\
C 6 / 1 C / 76 \\
C 7 / 07 / 76 \\
C E / 02 / 76 \\
C 8 / 25 / 76 \\
11 / 05 / 76\end{array}$ & $\begin{array}{l}65 / 05 / 76 \\
C 6 / 26 / 76 \\
C 7 / 24 / 76 \\
C 8 / 17 / 76 \\
08 / 30 / 76 \\
11 / 30 / 76\end{array}$ & $\begin{array}{l}\text { FLEL LCADING; STARTLP \& TEST PROGRAN } \\
\text { IASTRUMENT FAILURE \& MAINTEAANCE } \\
\text { TUBE REFAIR \& CTHER MAINTENANCE } \\
\text { REPAIR \& INSTALLATICA CF FEEChATER FLMFS } \\
\text { MAINTENANCE OUTAGE } \\
\text { FEECHATEF VIERATICN \& FCFTUFEC CRAIA LINE }\end{array}$ \\
\hline
\end{tabular}




\begin{tabular}{|c|c|c|c|c|c|c|c|}
\hline NWT & MWE-NET & $C R I T-L$ & ELECTRIC & $D C W N-\#$ & CHN-CATE & LP-DATE & REASON \\
\hline$C<40.0$ & 72.0 & $c 9 / 62$ & $12 / 62$ & $\begin{array}{l}1 \\
2 \\
3 \\
4 \\
5 \\
6 \\
7 \\
8 \\
9 \\
16 \\
11 \\
12 \\
13 \\
14 \\
15 \\
16 \\
17 \\
18 \\
19 \\
20 \\
21 \\
22 \\
23 \\
24 \\
25 \\
26 \\
27 \\
28 \\
25 \\
30\end{array}$ & 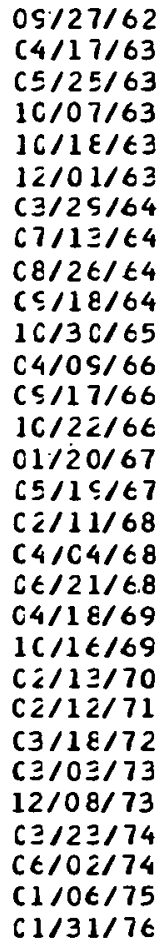 & $\begin{array}{l}12 / 08 / 62 \\
C 5 / 16 / 63 \\
C 8 / 26 / 63 \\
10 / 16 / 63 \\
10 / 23 / 63 \\
12 / 21 / 63 \\
05 / 21 / 64 \\
08 / 05 / 64 \\
09 / 15 / 64 \\
09 / 03 / 65 \\
11 / 04 / 65 \\
05 / 07 / 66 \\
10 / 21 / 66 \\
11 / 08 / 66 \\
02 / 09 / 67 \\
66 / 14 / 67 \\
03 / 15 / 68 \\
C 4 / 09 / 68 \\
07 / 16 / 68 \\
05 / 05 / 69 \\
10 / 20 / 69 \\
c 3 / 25 / 70 \\
03 / 13 / 71 \\
05 / 13 / 72 \\
C 4 / 15 / 73 \\
01 / 11 / 74 \\
C 5 / 05 / 74 \\
c 7 / 27 / 74 \\
06 / 07 / 75 \\
67 / 27 / 76\end{array}$ & 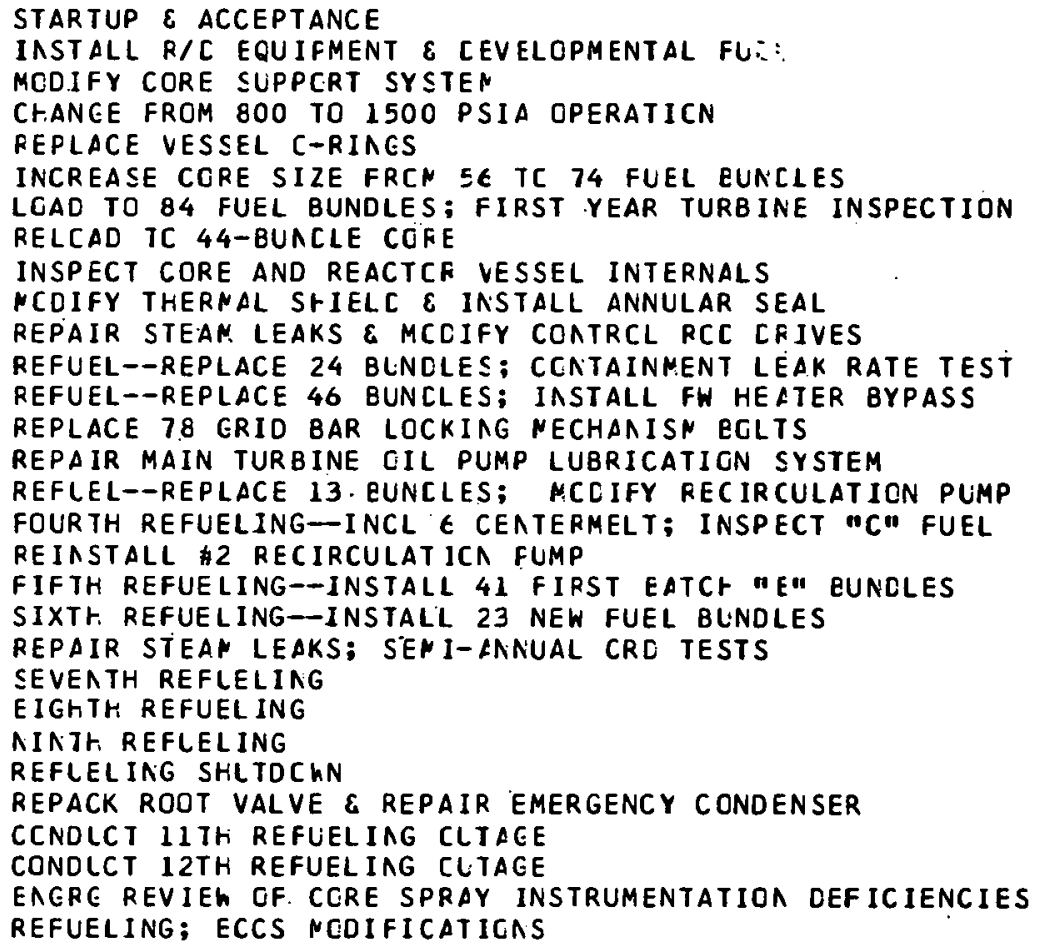 \\
\hline
\end{tabular}


BRCWRS FERRY NUCLEAR FEWER FLANT UNIT 1

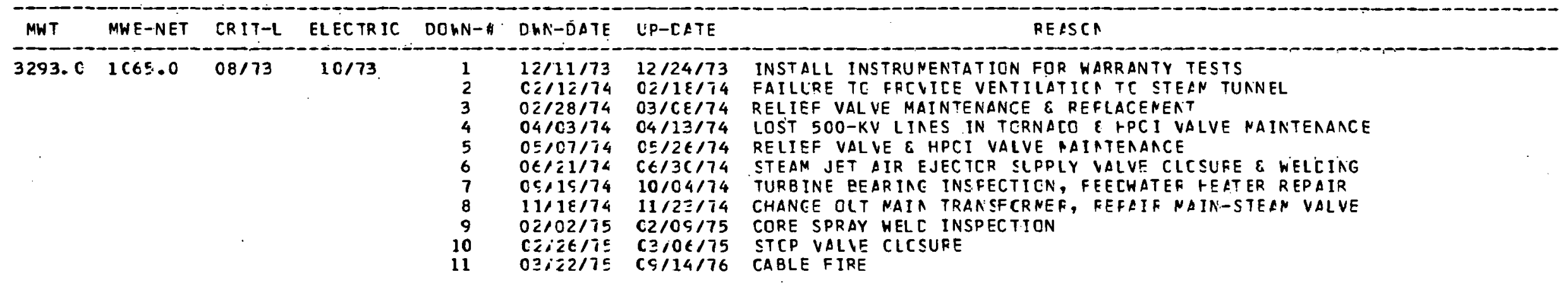


BRCWNS FERRY NUCLEAR PCWER FLANT UN $1 T 2$

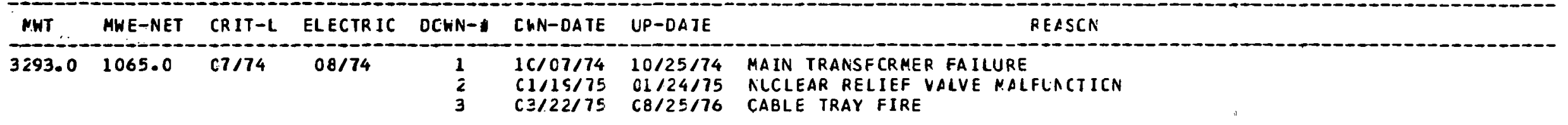


BRUNSWICK STEAM ELECTRIC PLANT INIT 2

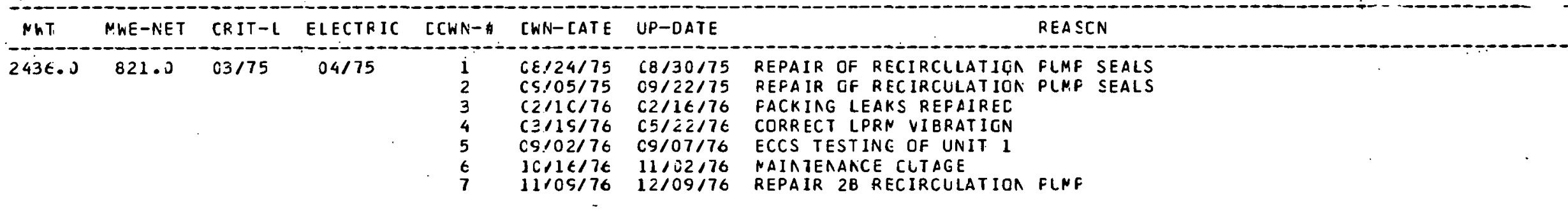


CALVERT CLIFFS NUCLEAR PCWER. PLANT LAIT 1

\begin{tabular}{|c|c|c|c|c|c|c|c|}
\hline \multicolumn{8}{|c|}{ ELECTRIC } \\
\hline $\begin{array}{l}\text { NhT } \\
2576 .-\end{array}$ & 845.0 & $10 / 74$ & $12 / 74$ & $\begin{array}{l}1 \\
2 \\
3 \\
4\end{array}$ & $\begin{array}{l}15 / 12 / 75 \\
08 / 11 / 75 \\
(4 / 1 C / 76 \\
12 / 31 / 76\end{array}$ & $\begin{array}{l}C 5 / 18 / 75 \\
08 / 22 / 75 \\
C 4 / 22 / 76\end{array}$ & $\begin{array}{l}\text { LCSS GF FEEC PUMP \& SUBSEGUENT WATER HAMMER IN FEED SYS } \\
\text { LEAK IN REACTOR COOL ING SYSTEM } \\
\text { LEAK RATE CETERMINATION CF CCATAINMENT PIFING PENETR } \\
\text { REFUELING }\end{array}$ \\
\hline
\end{tabular}


CCNALD C COCK PLANT LNIT I

\begin{tabular}{|c|c|c|c|c|c|c|c|}
\hline NWT & MHE-NET & $C R I T-L$ & ELECTRIC & CCWN-\# & $\operatorname{CHN}-[A T E$ & UP-DATE & REA SON \\
\hline 3250.0 & $1 C 54.0$ & $01 / 75$ & $02 / 75$ & $\begin{array}{l}1 \\
2 \\
3 \\
4 \\
5 \\
6\end{array}$ & $\begin{array}{l}C 2 / 18 / 75 \\
C 7 / 03 / 75 \\
1 C / 31 / 75 \\
04 / 12 / 76 \\
09 / 10 / 76 \\
12 / 31 / 76\end{array}$ & $\begin{array}{l}03 / 31 / 75 \\
07 / 23 / 75 \\
C 9 / 25 / 75 \\
C 5 / 1 C / 76 \\
09 / 19 / 76 \\
1,1\end{array}$ & $\begin{array}{l}\text { MAINTENANCE GUTAGE } \\
\text { MAIN CCNCENSER. LEAK } \\
\text { INSFECT MAIA UNIT TRANSFCRNER; CTHER MAINTENANCE } \\
\text { SLRV TEST; S-G MCO; EDCY CLRREAT TEST; MAINTENANCE } \\
\text { INSPECT MAIN UNIT TRANSFCRMERS; CTHER MAINTENANCE } \\
\text { REFLELING }\end{array}$ \\
\hline
\end{tabular}




\begin{tabular}{|c|c|c|c|c|c|c|c|}
\hline NHT & MHE-NET & CRIT-L & ELECTRIC & COWN- & CWN-CATE & LP-DATE & REASCA \\
\hline 2381.0 & 778.0 & $c 2 / 74$ & $05 / 74$ & $\begin{array}{l}1 \\
2 \\
3 \\
4 \\
5 \\
6\end{array}$ & $\begin{array}{l}C 2 / 03 / 75 \\
C S / 27 / 75 \\
12 / 10 / 75 \\
C 1 / 07 / 76 \\
C 5 / 0 \& / 76 \\
C S / 1 \varepsilon / 76\end{array}$ & $\begin{array}{l}02 / 05 / 75 \\
11 / 01 / 75 \\
12 / 18 / 75 \\
01 / 1 \varepsilon / 76 \\
05 / 24 / 76 \\
11 / 12 / 76\end{array}$ & $\begin{array}{l}\text { LLTRASCNIC TESTING CF FIFINE } \\
\text { PLUG LCHER CCRE PLATE EYFASS FLCh CF HCLES } \\
\text { CENERATOR EXCITER FAILURE } \\
\text { REPAIR/REDESIGN CFFGAS SYSTEN } \\
\text { REPLACEMENT GF GENERATCF EXCITER E TCRLS REPAIR } \\
\text { REFUELINE }\end{array}$ \\
\hline
\end{tabular}


CRESDEN NUCLEAR PCWER STATICA UNIT I

\begin{tabular}{|c|c|c|c|c|c|c|c|}
\hline MWT & MWE-NET & CRI T-L & ELECTRIC & DOWN-\# & DHA-CATE & UP-DETE & REASON \\
\hline $070 \mathrm{C} . \mathrm{C}$ & $\mathrm{C} 2 \mathrm{CC} .0$ & $10 / 5 s$ & $04 / 60$ & $\begin{array}{l}1 \\
2 \\
3 \\
4 \\
5 \\
6 \\
7 \\
8 \\
9 \\
10 \\
11 \\
12 \\
12 \\
14 \\
15 \\
16 \\
17 \\
18 \\
19 \\
20 \\
21 \\
22 \\
23 \\
24 \\
25 \\
26\end{array}$ & 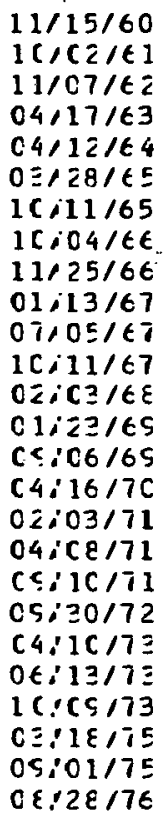 & $\begin{array}{l}C \epsilon / 02 / 61 \\
12 / 0 \epsilon / \epsilon 1 \\
C 2 / E 1 / \epsilon 3 \\
C 4 / 25 / 63 \\
0 \epsilon / C S / \epsilon 4 \\
C 5 / 2 C / \epsilon 5 \\
10 / 17 / 65 \\
10 / C C / \epsilon 6 \\
11 / 3 C / \epsilon 6 \\
05 / 28 / \epsilon 7 \\
C 7 / / C / 67 \\
11 / C 4 / 67 \\
C 6 / 02 / \epsilon 8 \\
02 / C 2 / \epsilon S \\
12 / 31 / 69 \\
04 / 2 \epsilon / 70 \\
C 2 / 11 / 71 \\
04 / 16 / 71 \\
02 / C 2 / 72 \\
11 / 0 \equiv / 72 \\
C 4 / 15 / 73 \\
C \epsilon / 1 C / 73 \\
C 7 / 05 / 74 \\
04 / 0 S / 75 \\
01 / 25 / 76 \\
0 S / 11 / 76\end{array}$ & 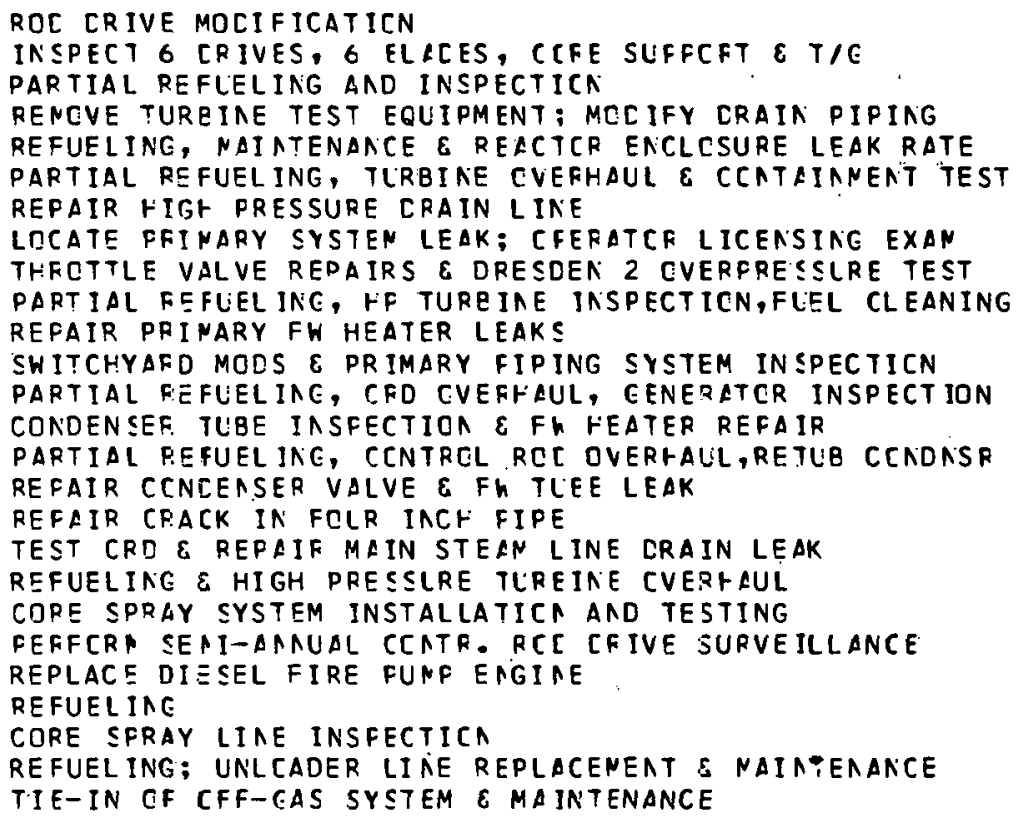 \\
\hline
\end{tabular}


CRESDEN NUCLEAR PCWER STATICN UNIT 2

\begin{tabular}{|c|c|c|c|c|c|c|c|}
\hline MWT & MWE-NET & CRI T-L & ELECTRIC & DCHN-\# & CHA-CATE & L:P-CATE & REASON \\
\hline $2527 . \mathrm{C}$ & 809.0 & $01 / 7 \mathrm{C}$ & $C 4 / 7 C$ & $\begin{array}{l}1 \\
2 \\
3 \\
4 \\
5 \\
6 \\
7 \\
8 \\
9 \\
10 \\
11 \\
12 \\
13 \\
14 \\
15 \\
16 \\
17 \\
18 \\
19\end{array}$ & 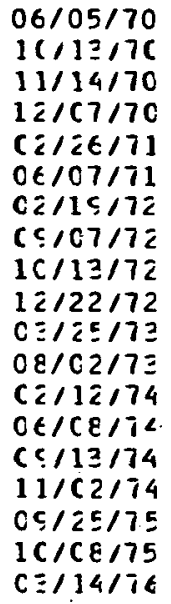 & $\begin{array}{l}C 8 / 11 / 70 \\
10 / 25 / 70 \\
1 / / 2 C / 70 \\
12 / 27 / 70 \\
C E / 27 / 71 \\
C E / 1 Z / 71 \\
C 5 / 10 / 72 \\
C S / Z 1 / 72 \\
11 / 07 / 72 \\
12 / 31 / 72 \\
C 4 / C Z / 73 \\
C 8 / 13 / 73 \\
03 / / 7 / 74 \\
C E / 15 / 74 \\
10 / 107 / 74 \\
C 5 / 1 C / 75 \\
1 C / C 1 / 75 \\
10 / 14 / 75 \\
C E / 24 / 76\end{array}$ & 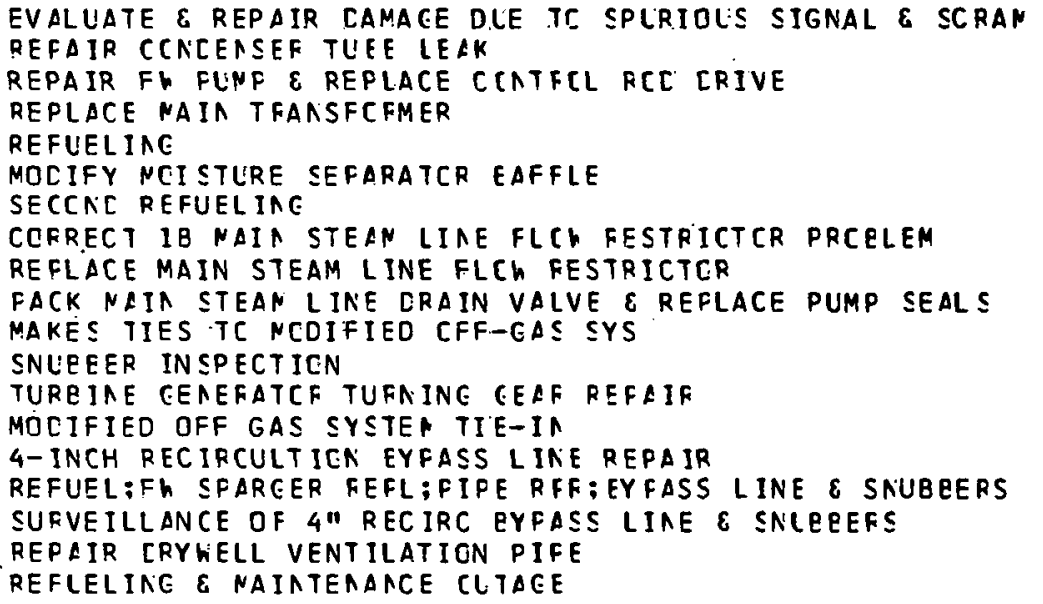 \\
\hline
\end{tabular}




\begin{tabular}{|c|c|c|c|c|c|c|c|}
\hline NWT & MWE-NET & CRIT-L & ELECTRIC & CCWN-\# & CWR - CATE & L:P-DATE & REASON \\
\hline $2527 . \mathrm{C}$ & 809.0 & $\mathrm{Cl} / 7 \mathrm{I}$ & $07 / 71$ & $\begin{array}{r}1 \\
2 \\
3 \\
4 \\
5 \\
6 \\
7 \\
8 \\
9 \\
10 \\
11 \\
12 \\
12 \\
14\end{array}$ & $\begin{array}{l}C \& ; 25 / 71 \\
C \subseteq: C S / 71 \\
1 C: 18 / 71 \\
12: 08 / 71 \\
C 4: 14 / 72 \\
11: 18 / 72 \\
C 7: 31 / 73 \\
C 1 / 17 / 74 \\
C 3: 11 / 74 \\
C E: 24 / 74 \\
C S / 2 C / 74 \\
02: 18 / 75 \\
04 / 16 / 75 \\
C S 115 / 76\end{array}$ & $\begin{array}{l}09 / 06 / 71 \\
C 9 / 18 / 71 \\
10 / 28 / 71 \\
12 / 28 / 71 \\
C 4 / 25 / 72 \\
11 / 24 / 72 \\
C 8 / 1 C / 73 \\
C 1 / 24 / 74 \\
06 / 06 / 74 \\
C 7 / 02 / 74 \\
65 / 28 / 74 \\
C 2 / 25 / 75 \\
C 9 / 19 / 75 \\
11 / 05 / 76\end{array}$ & 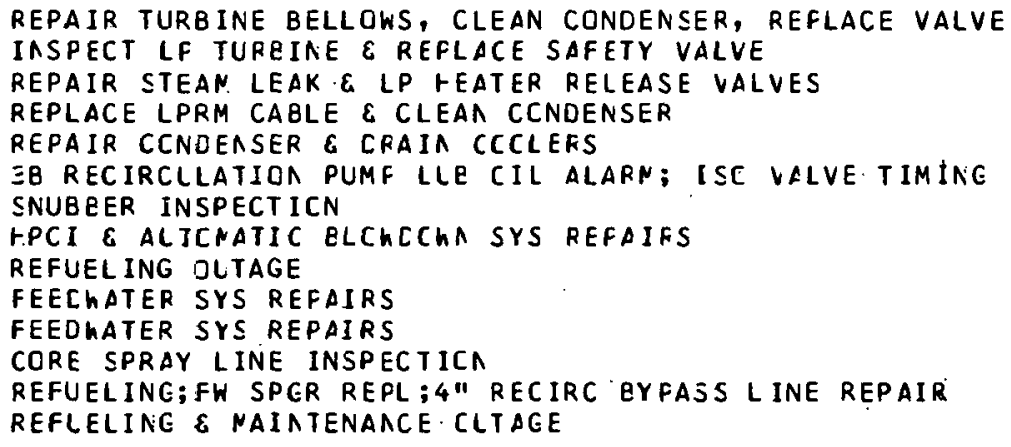 \\
\hline
\end{tabular}


JAMES A FITZPATRICK NLCLEAR FOHER PLANT

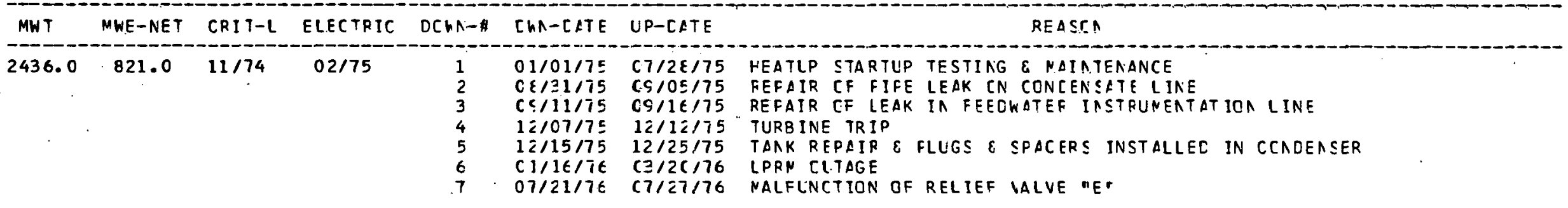


FCRT CALHCUN STATICN UNIT I

\begin{tabular}{|c|c|c|c|c|c|c|c|}
\hline Mn'T & MWE-NET & CR I T-L & ELECTR IC & $D C W N=A$ & CHA-CATE & UP-DATE & REA SON \\
\hline $1<20.0$ & $457 . C$ & $\mathrm{C} 8 / 73$ & $0 \varepsilon / 73$ & $\begin{array}{l}1 \\
2 \\
3 \\
4 \\
5 \\
6 \\
7 \\
8\end{array}$ & $\begin{array}{l}03 / 07 / 74 \\
C \equiv / 25 / 74 \\
C 4 / 17 / 74 \\
11 / 05 / 74 \\
C 2 / 01 / 75 \\
C 5 / 16 / 75 \\
C 2 / 21 / 76 \\
1 C / 01 / 76\end{array}$ & $\begin{array}{l}03 / 15 / 74 \\
64 / 11 / 74 \\
C 4 / 2 S / 74 \\
11 / 17 / 74 \\
C 5 / 05 / 75 \\
10 / 06 / 75 \\
03 / 25 / 76 \\
12 / 1 C / 76\end{array}$ & $\begin{array}{l}\text { TESTING } E \text { INSTALLATION OF MAIN STEAM LINE RESTRAINTS } \\
\text { NAIA SIEAN LINE ISCLATICA VALVE REFAIRS. } \\
\text { AIR PIPING SYS E REAC CCCLANT PUMPS SEALS REPAIR } \\
\text { REACTCR CCCLANT SYS CHENICALLY CLEANED } \\
\text { REFUELING } \\
\text { FEPAIR REACTCR CCOLANT FLNP SEAL } \\
\text { REPLACEMENT CF. INCORE CETECTGR } \\
\text { REFLELING }\end{array}$ \\
\hline
\end{tabular}




\begin{tabular}{|c|c|c|c|c|c|c|c|}
\hline MWT & NWE-NET & CRI I-L & ELECTRIC & CCWN-A & {$[W A-C A T E$} & UP-CDATE & REASCA \\
\hline 0841.7 & 330.0 & $01 / 74$ & $12 / 76$ & $\begin{array}{l}1 \\
2 \\
2 \\
4 \\
5\end{array}$ & $\begin{array}{l}0 E / C 1 / 7 E \\
C E / C 3 / 7 t \\
C C / 21 / 7 t \\
1 C / 25 / 7 t \\
11 / 23 / 7 t\end{array}$ & $\begin{array}{l}(7 / C 1 / 76 \\
C \varepsilon / 24 / 76 \\
1 C / 15 / 76 \\
11 / 15 / 76 \\
12 / 05 / 76\end{array}$ & $\begin{array}{l}\text { CAELE SEFARATICN E ELEC SEGREGATICA PRCELENS } \\
\text { REFLACE IC IELILN CIRCLLATCR } \\
\text { REFAIR SH STEAN EYFASS SC VALVE } \\
\text { REPAIR NSIV PACKING E HYC CIL SYSTEN } \\
\text { REVIEW DF CIRCUITRY }\end{array}$ \\
\hline
\end{tabular}


RCEERT EMMETT GINAA NUCLEAR PCWER PLANT LNIT:

\begin{tabular}{|c|c|c|c|c|c|c|c|}
\hline NWT & MWE-NET & CRIT-L & ELECTRIC & $D C W N-\#$ & CHA-CATE & LP-CATE & REASON \\
\hline$\varepsilon 20 . \mathrm{C}$ & .1490 .0 & $11 / 69$ & $12 / 69$ & $\begin{array}{l}1 \\
2 \\
3 \\
4 \\
5 \\
6 \\
7 \\
8 \\
9 \\
1 C \\
11 \\
12 \\
13 \\
14 \\
15 \\
16 \\
17 \\
1 \varepsilon \\
19\end{array}$ & 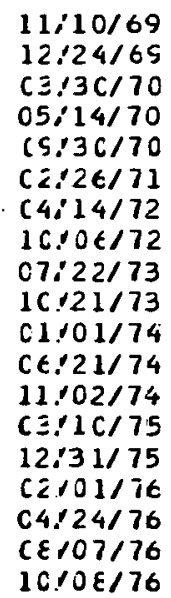 & $\begin{array}{l}11 / 27 / 69 \\
01 / 17 / 70 \\
C 4 / 24 / 70 \\
06 / 19 / 70 \\
10 / 15 / 70 \\
C 5 / 13 / 71 \\
c 6 / 24 / 72 \\
11 / 10 / 72 \\
07 / 31 / 73 \\
1 C / 27 / 73 \\
c 4 / 25 / 74 \\
06 / 26 / 74 \\
11 / 13 / 74 \\
C 5 / 15 / 75 \\
01 / 11 / 76 \\
c 4 / 16 / 76 \\
05 / 07 / 76 \\
c 9 / 03 / 76 \\
1 J / 25 / 76\end{array}$ & 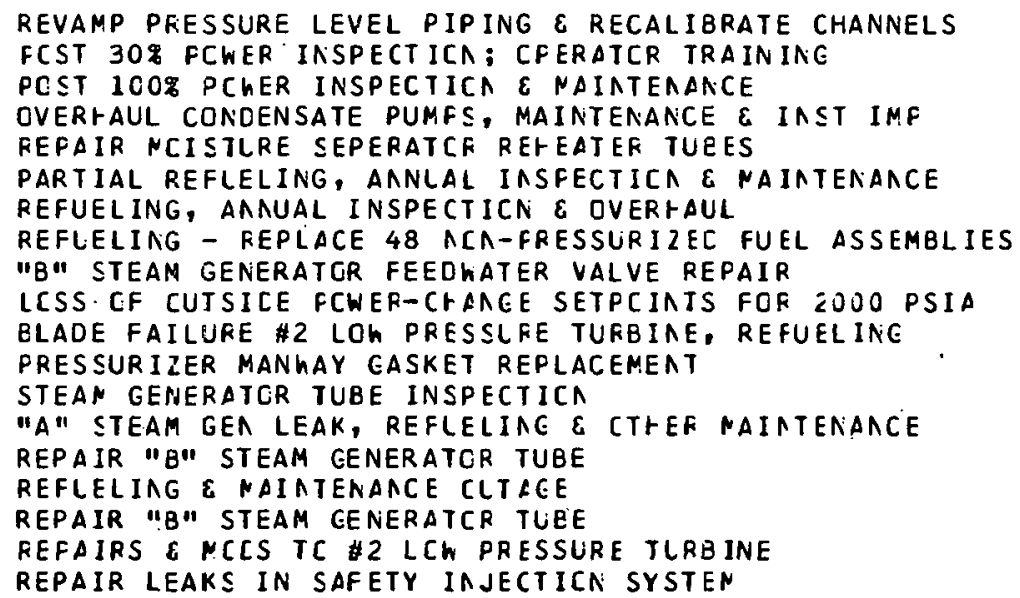 \\
\hline
\end{tabular}




\begin{tabular}{|c|c|c|c|c|c|c|c|}
\hline NWT & MWE-NET & $C R I T-L$ & ELECTRIC & $D C h N-A$ & CWN-CATE & UP-OATE & REASON \\
\hline $1 \varepsilon 25 . \mathrm{C}$ & 575.0 & $07 / 67$ & $08 / 67$ & $\begin{array}{l}1 \\
2 \\
3 \\
4 \\
5 \\
6 \\
7 \\
8 \\
9 \\
16 \\
11 \\
12 \\
13 \\
14 \\
15 \\
16 \\
17 \\
18\end{array}$ & $\begin{array}{l}C 8 / 07 / 67 \\
C E / 21 / 67 \\
1 C / 05 / 67 \\
10 / 25 / 67 \\
11 / 2 C / 67 \\
C 1 / 12 / 68 \\
C 3 / 01 / 68 \\
C 3 / 15 / 68 . \\
C 3 / 2 S / 68 \\
C 4 / 11 / 69 \\
C 4 / 17 / 70 \\
C 4 / 16 / 71 \\
C E / 1 C / 72 \\
C E / 02 / 73 \\
C 7 / 08 / 73 \\
C 3 / 23 / 74 \\
C 5 / 17 / 75 \\
05 / 18 / 76\end{array}$ & $\begin{array}{l}08 / 15 / 67 \\
10 / 02 / 67 \\
16 / 10 / 67 \\
10 / 30 / 67 \\
11 / 28 / 67 \\
C 1 / 23 / 68 \\
03 / 12 / 68 \\
03 / 27 / 68 \\
04 / 15 / 68 \\
05 / 11 / 69 \\
06 / 26 / 70 \\
05 / 26 / 71 \\
07 / 14 / 72 \\
06 / 17 / 73 \\
12 / 14 / 73 \\
04 / 20 / 74 \\
07 / 01 / 75 \\
07 / 19 / 76\end{array}$ & 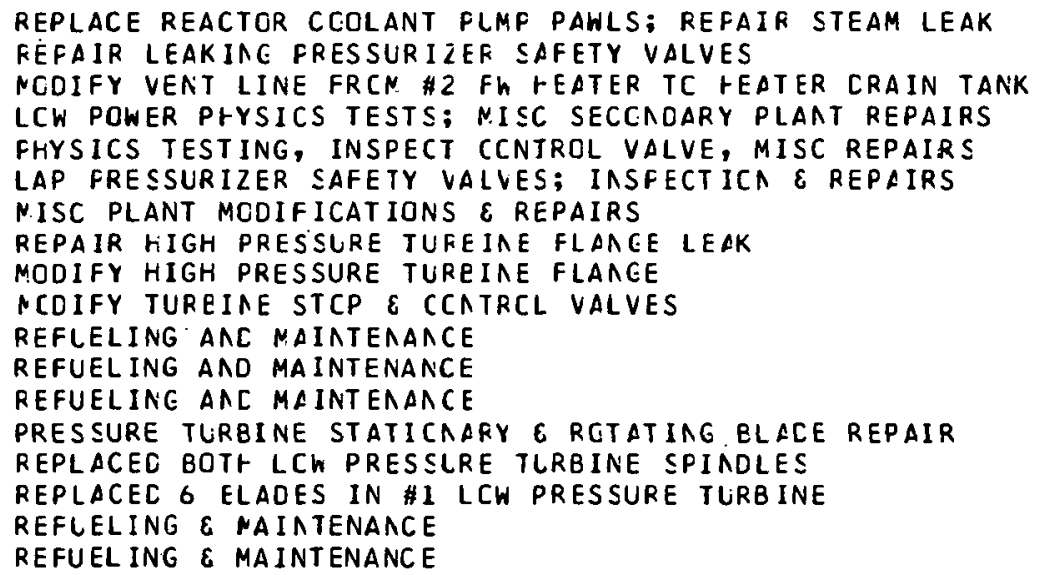 \\
\hline
\end{tabular}


ECWIN I HATCH. NUCLEAR FLANT UNIT I

\begin{tabular}{|c|c|c|c|c|c|c|c|}
\hline NWT & MWE-NET & CRIT-L & ELECTR IC & CCWN-t & CHN-CATE & UP-DATE & REA SON \\
\hline 2436.0 & $786 . \mathrm{C}$ & $\mathrm{C} 9 / 74$ & $11 / 74$ & $\begin{array}{l}1 \\
2 \\
3 \\
4 \\
5\end{array}$ & $\begin{array}{l}02 / 1) 2 / 75 \\
(4 / 1 \subseteq / 75 \\
11 / 1 \in / 75 \\
(3 / 27 / 76 \\
C \leqslant / 2 \subseteq / 76\end{array}$ & $\begin{array}{l}02 / 11 / 75 \\
64 / 30 / 75 \\
12 / 24 / 75 \\
04 / 22 / 76 \\
06 / 05 / 76\end{array}$ & $\begin{array}{l}\text { RADICGRAPH WELOS IN DRYWELL } \\
\text { SEHC CIL LEAK;MAIAT TUFEIAE. SV \& CIV SCREEA REMEVAL } \\
\text { IMPLEMENT LFRM VIBRATICA FIX } \\
\text { PCDIFY LFCI SYSTEM \& REFLIR MSIV } \\
\text { REPLACE "B" N-G SET CRIVE MCTCF }\end{array}$ \\
\hline
\end{tabular}




\begin{tabular}{|c|c|c|c|c|c|c|c|}
\hline NhT & MWE-NET & CRIT-L & ELECTRIC & {$[C W N-\#$} & CWN-CATE & UP-DATE & REASCN \\
\hline 220.0 & $E 2.0$ & $C 2 / \leftarrow 3$ & $04 / 6 ?$ & $\begin{array}{l}1 \\
2 \\
3 \\
4 \\
5 \\
6 \\
7 \\
8 \\
5 \\
10 \\
11 \\
12 \\
13 \\
14 \\
15 \\
16 \\
17 \\
18 \\
19 \\
20 \\
21 \\
22\end{array}$ & $\begin{array}{l}1 C / 26 / 63 \\
C 1 / 1 S / \epsilon 4 \\
0 \varepsilon / 16 / 64 \\
C \subseteq / 20 / \epsilon 5 \\
C 1 / 14 / 6 \epsilon \\
11 / 1 \varepsilon / 66 \\
C 3 / 2 S / 67 \\
1 C / 04 / 67 \\
1 C / 05 / 68 \\
C \epsilon / 14 / \epsilon 9 \\
04 / 17 / 70 \\
C 7 / 17 / 7 C \\
C 1 / 0 \varepsilon / 71 \\
C \epsilon / 06 / 71 \\
C \varepsilon / 25 / 72 \\
10 / 0 \epsilon / 72 \\
C S / 01 / 73 \\
1 C / 3 C / 74 \\
C 5 / 30 / 75 \\
C 7 / 14 / 75 \\
C 3 / 17 / 7 \epsilon \\
07 / 02 / 76\end{array}$ & $\begin{array}{l}11 / 17 / 63 \\
C 2 / C 2 / 64 \\
09 / 13 / 64 \\
12 / 01 / 65 \\
01 / 2 C / 66 \\
12 / 20 / 66 \\
C 4 / 05 / 67 \\
10 / 28 / 67 \\
10 / 26 / 68 \\
C 7 / 20 / 69 \\
05 / 09 / 70 \\
C E / C 7 / 770 \\
C 1 / 14 / 71 \\
08 / 26 / 71 \\
10 / J 6 / 72 \\
10 / 23 / 72 \\
10 / 02 / 73 \\
12 / 25 / 74 \\
c 7 / 08 / 75 \\
c 7 / 22 / 75 \\
C 3 / 26 / 76 \\
/ / 1 /\end{array}$ & 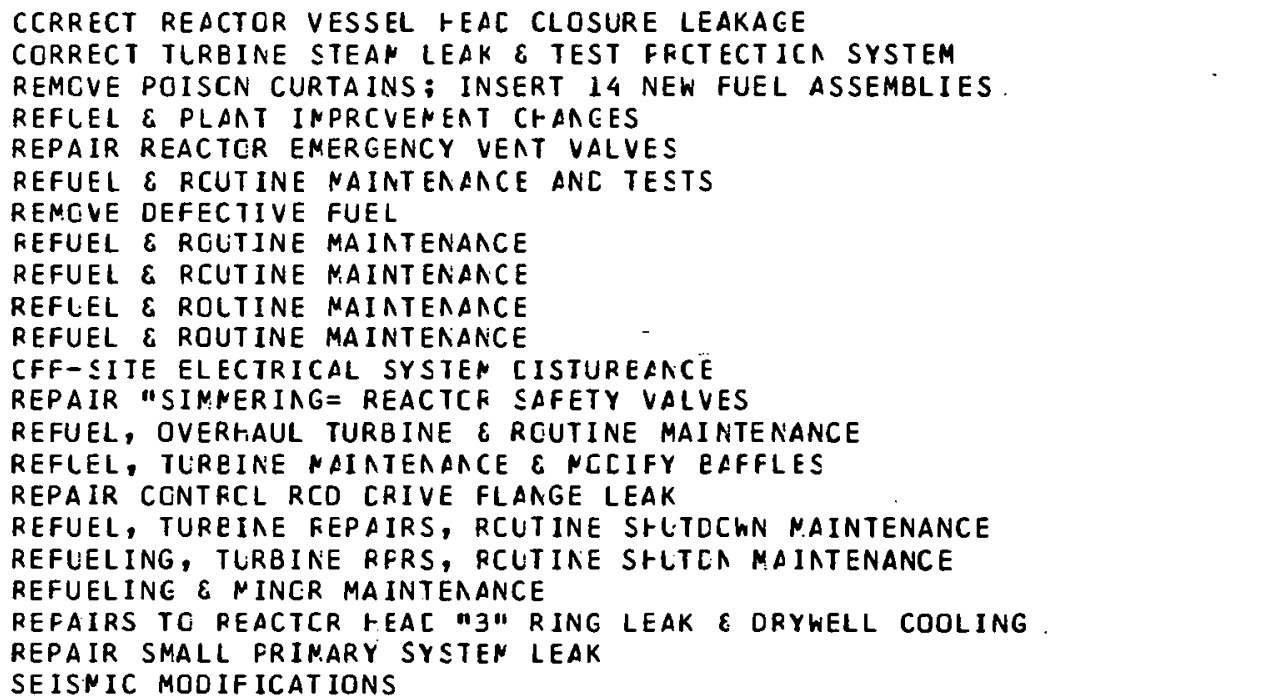 \\
\hline
\end{tabular}


INDIAN POINT STATION UNIT I

\begin{tabular}{|c|c|c|c|c|c|c|c|}
\hline MH!T & MWE-NET & CRIT-L & ELEC-RIC & $D . O h N-\#$ & CHM-CATE & UF-DATE & REASON \\
\hline $0 \notin 15.0$ & $2 \in 5 . C$ & $\mathrm{C} 8 / \in 2$ & $C 9 / t 2$ & $\begin{array}{l}1 \\
2 \\
3 \\
4 \\
5 \\
6 \\
7 \\
8 \\
9 \\
10 \\
11 \\
12 \\
13 \\
14 \\
15 \\
16 \\
17 \\
18 \\
19 \\
20 \\
.11 \\
22 \\
23 \\
24 \\
25 \\
26 \\
27 \\
28 \\
29 \\
30 \\
31\end{array}$ & 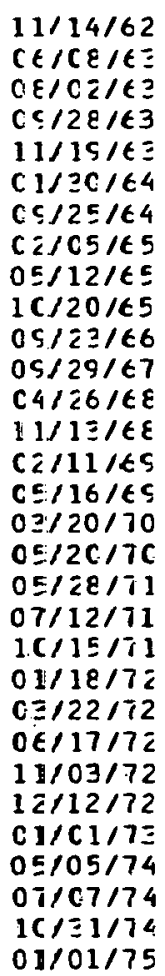 & 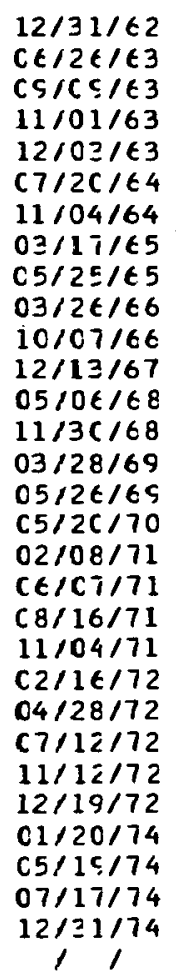 & 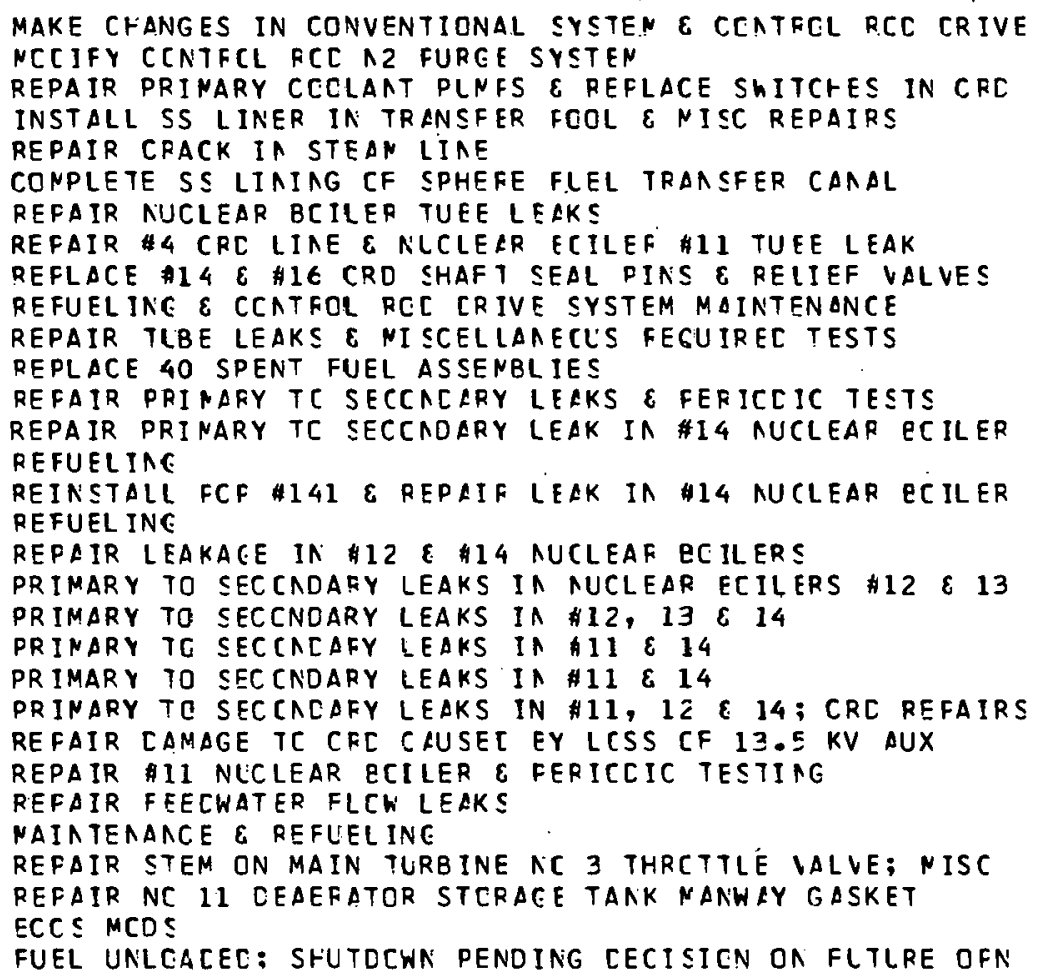 \\
\hline
\end{tabular}


INDIAN PEIAT STATIEA UNIT E

\begin{tabular}{|c|c|c|c|c|c|c|c|}
\hline MWT & MHE-NET & CR I I-L & ELEC TR IC & DOHN-H & CHA-DATE & LP-CATE & REASCA \\
\hline $2758 . c$ & $873 . \mathrm{C}$ & $05 / 73$ & $06 / 73$ & $\begin{array}{l}1 \\
2 \\
3 \\
4 \\
5 \\
6 \\
7 \\
8 \\
9 \\
10 \\
11 \\
12 \\
12 \\
14 \\
15\end{array}$ & 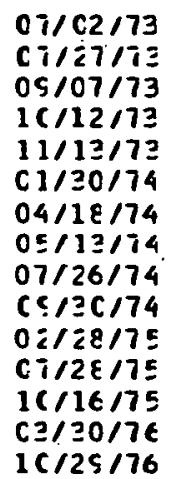 & $\begin{array}{l}c 7 / 10 / 73 \\
\times \varepsilon / 04 / 73 \\
C 9 / 21 / 73 \\
11 / 13 / 73 \\
01 / 26 / 74 \\
C 2 / 22 / 74 \\
04 / 29 / 74 \\
05 / 21 / 74 \\
C 8 / 07 / 74 \\
10 / 12 / 74 \\
C 4 / 04 / 75 \\
C 8 / 11 / 75 \\
10 / 31 / 75 \\
C 5 / 27 / 76 \\
12 / 10 / 76\end{array}$ & 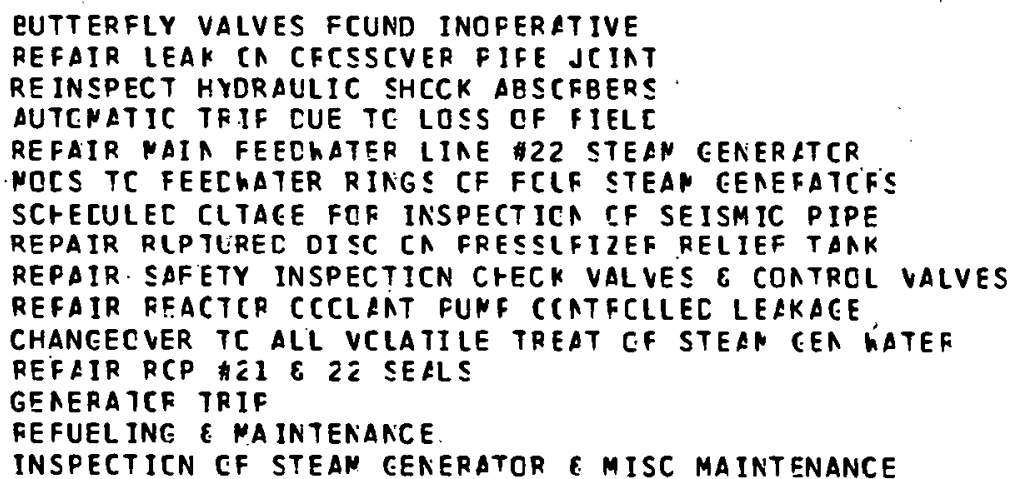 \\
\hline
\end{tabular}


INDIAN FCINT STATION LNIT Z

\begin{tabular}{|c|c|c|c|c|c|c|c|}
\hline MWT & MWE-NET & CRIT-L & ELECTRIC & CChN-\# & ChN-CATE & LP-CATE & \\
\hline 15 & 873.0 & $04 / 76$ & $04 / 76$ & $\frac{1}{2}$ & $05 / 15 / 7 t$ & $\begin{array}{l}\text { C5/22/76 } \\
10 / 02 / 76\end{array}$ & NAINTENANCE CUTAEE \\
\hline
\end{tabular}

RE $A S O N^{\prime}$ 
KEGAUNEE NUCLEAR FChEF PLANT UNIT 1

\begin{tabular}{|c|c|c|c|c|c|c|c|}
\hline NWT & NWE-NET & $C R I T-L$ & ELECTRIC & CCWN-G & ChN-CATE & LP-DATE & FEASCA \\
\hline $1<50.0$ & 535.0 & $\mathrm{C} 3 / 74$ & $C 4 / 74$ & $\begin{array}{l}1 \\
2 \\
3 \\
4 \\
5 \\
6\end{array}$ & $\begin{array}{l}C E / 2 \varepsilon / 74 \\
C S / 2 C / 74 \\
C S / 1 / 75 \\
1 C / 2 \epsilon / 75 \\
C 2 / 14 / 76 \\
C S / C S / 76\end{array}$ & $\begin{array}{l}07 / 10 / 74 \\
10 / 15 / 74 \\
09 / 19 / 75 \\
11 / 02 / 75 \\
C 4 / 11 / 76 \\
C 5 / 18 / 76\end{array}$ & $\begin{array}{l}\text { FRINARY \& SECCNCARY SYSTEM MINOR MAINTENANCE } \\
\text { REQLIRED STEAN GENEFATCF TLEE INSFECTICA } \\
\text { REPAIR STEAM GENERATOR \& PRESSURIZER MANHAY LEAKS } \\
\text { CVERFALL MAIA STEAM ISCLATICA VALVES } \\
\text { REFLELING } \\
\text { SEAL REFLACENEAT CN FEACTCR VESSEL FEAC }\end{array}$ \\
\hline
\end{tabular}


LACROSSE (GENOA) NUCLEAR GENERATIAG STATICA

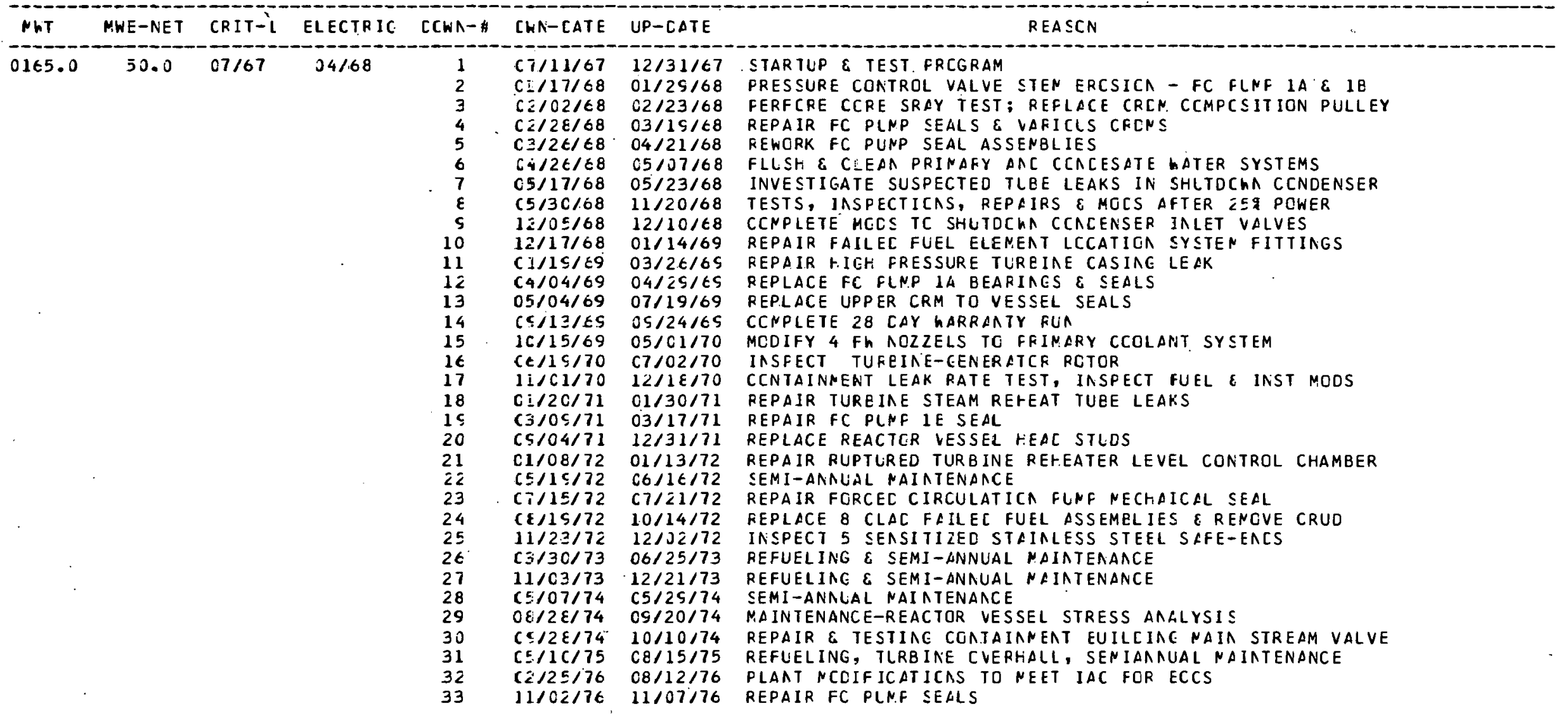


MAINE YANKEE ATOM IC FCWER PLANT

\begin{tabular}{|c|c|c|c|c|c|c|c|}
\hline M.HT & MWE-NET & CR IT - L & ELECTRIC & $C C W N-A$ & ChN-CATE & UP-DATE & REASCA \\
\hline 2440.0 & .790 .0 & $10 / 72$ & $11 / 72$ & $\begin{array}{l}1 \\
2 \\
3 \\
4 \\
5 \\
6 \\
7 \\
2\end{array}$ & $\begin{array}{l}1 C / 23 / 72 \\
C</ 18 / 73 \\
06 / 29 / 73 \\
C \subseteq / 07 / 73 \\
C E / 28 / 74 \\
01 / 16 / 75 \\
C 5 / 02 / 75 \\
0 S / 03 / 76\end{array}$ & $\begin{array}{l}12 / 28 / 72 \\
C 3 / 12 / 73 \\
08 / 02 / 73 \\
05 / 20 / 73 \\
11 / 11 / 74 \\
01 / 22 / 75 \\
06 / 28 / 76 \\
C 9 / 14 / 76\end{array}$ & $\begin{array}{l}\text { STARTUP } \& \text { TEST PROGRAM } \\
\text { INSPECTION } \& \text { REFAIR CF LCW FFESSLFE TUFEINE } \\
\text { SCHECULE TURBINE/GENERATCR INSPECTION } \\
\text { GENERATCR ALIGNMAT \& EALANCIAE } \\
\text { FLEL REPLACEMENT } \\
\text { EXAMINE FEECWATER LINES } \\
\text { REFLELING } \\
\text { REPLACEMENT CF REACTCR FLMP SEAL ASSEMELIES }\end{array}$ \\
\hline
\end{tabular}


NILLSTCNE NUCLEAR FCWER STATION UNIT I

\begin{tabular}{|c|c|c|c|c|c|c|c|}
\hline MWT & MWE-NET & CRIT $T-L$ & ELECTRIC & $C C W N-A$ & CHR-CATE & LUP-DATE & REASON \\
\hline $2 \mathrm{C} 11.0$ & 652.0 & $10 / 70$ & $11 / 70$ & $\begin{array}{l}1 \\
2 \\
3 \\
4 \\
5 \\
6 \\
7 \\
8 \\
9 \\
10 \\
11 \\
12 \\
13 \\
14 \\
15 \\
16 \\
17 \\
18 \\
19 \\
20 \\
21\end{array}$ & 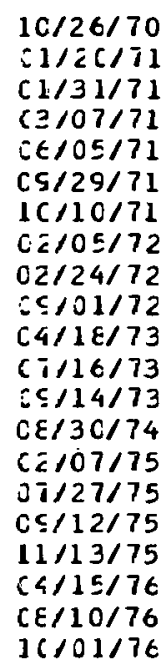 & $\begin{array}{l}11 / 29 / 70 \\
C 1 / 24 / 71 \\
02 / 11 / 71 \\
C 3 / 17 / 71 \\
c 6 / 11 / 71 \\
10 / 03 / 71 \\
10 / 24 / 71 \\
C 2 / 13 / 72 \\
03 / 01 / 72 \\
12 / 31 / 72 \\
c 7 / 16 / 73 \\
c 7 / 28 / 73 \\
c 9 / 22 / 73 \\
11 / 02 / 74 \\
C 2 / 15 / 75 \\
08 / 13 / 75 \\
10 / 21 / 75 \\
11 / 23 / 75 \\
C 4 / 20 / 76 \\
08 / 18 / 76 \\
12 / 01 / 76\end{array}$ & 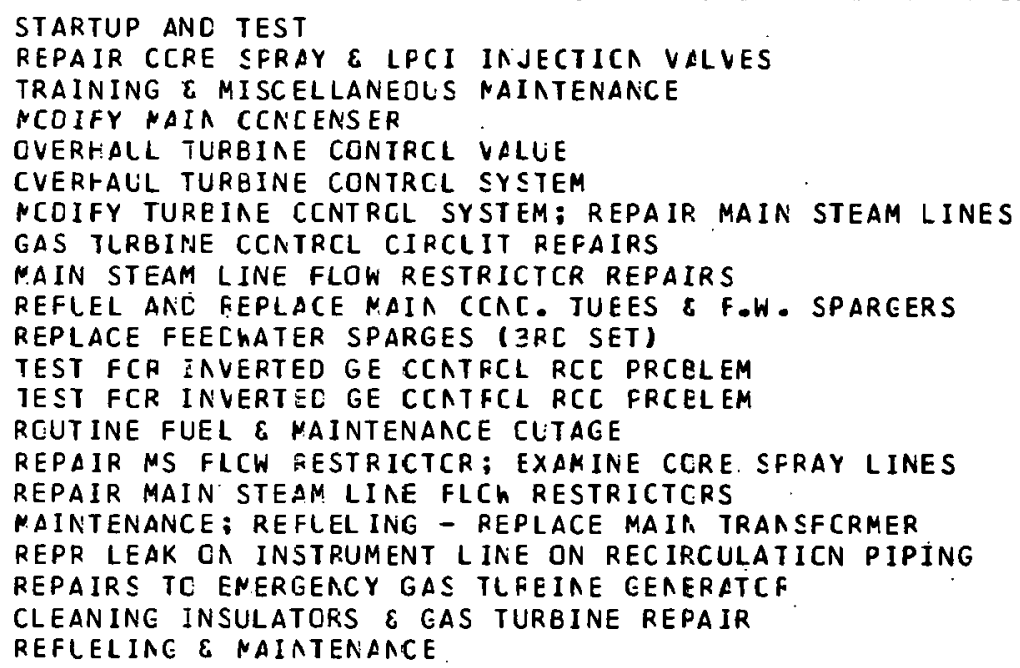 \\
\hline
\end{tabular}


millstcae nliclear fCher statica lait 2

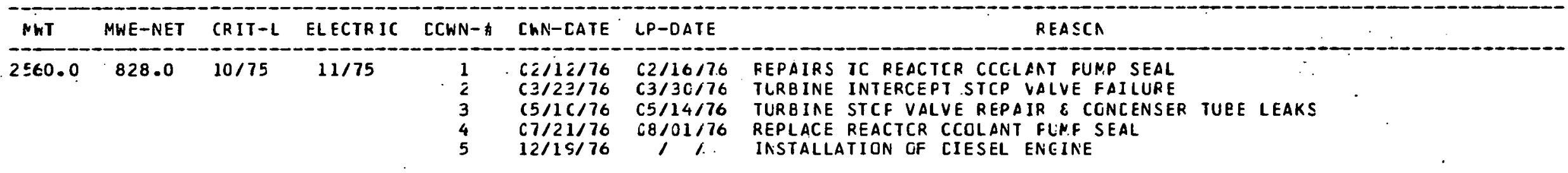


MCNTICELLC NUCLEAF GENERATINE FLAAT

\begin{tabular}{|c|c|c|c|c|c|c|c|}
\hline MWT & MWE-NET & CRIT-L & ELECTRIC & $C \mathrm{CWN}-\mathrm{A}$ & CHN-CATE & LP-DATE & REASCA \\
\hline 1670.0 & 545.0 & $12 / 70$ & $03 / 71$ & $\begin{array}{l}1 \\
2 \\
3 \\
4 \\
5 \\
6 \\
7 \\
8 \\
5 \\
10 \\
11 \\
12 \\
13 \\
14 \\
15 \\
16 \\
17\end{array}$ & 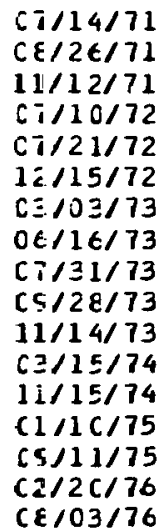 & $\begin{array}{l}c 7 / 2 t / 71 \\
c 9 / 0 / / 71 \\
01 / 31 / 72 \\
07 / 17 / 72 \\
c 8 / 02 / 72 \\
12 / 21 / 72 \\
C 5 / 15 / 73 \\
06 / 20 / 73 \\
c 8 / 10 / 73 \\
10 / 0 t / 73 \\
11 / 18 / 73 \\
c 5 / 21 / 74 \\
11 / 21 / 74 \\
C 2 / 01 / 75 \\
1 c / 31 / 75 \\
C 3 / 01 / 76 \\
c 5 / 09 / 76\end{array}$ & 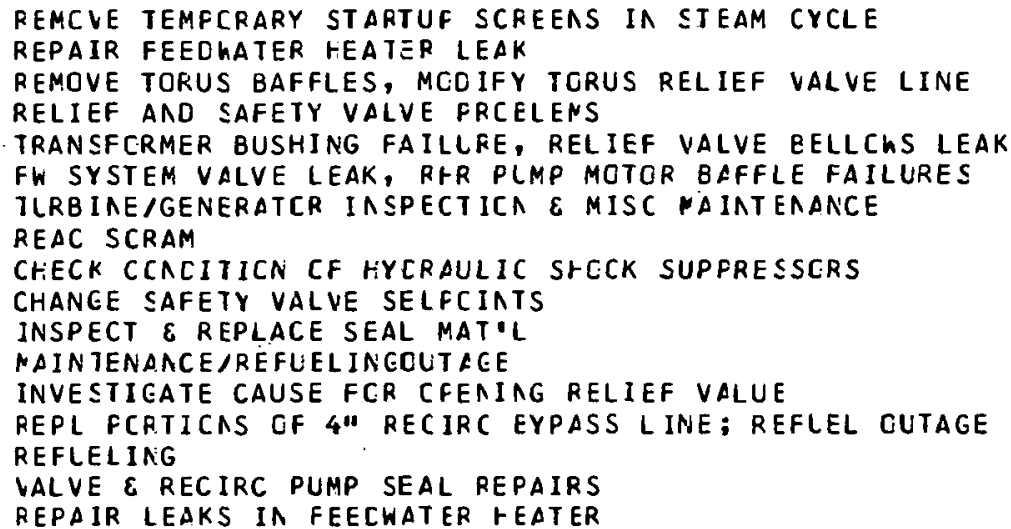 \\
\hline
\end{tabular}


NINE MILE POINT NLCLEAR STATION LNIT 1

\begin{tabular}{|c|c|c|c|c|c|c|c|}
\hline N.WT & MHE-NET & CR IT $\div \mathrm{L}$ & ELECTRIC & {$[C W N-A$} & CWN-CATE & UP-DATE & REA $S C N$ \\
\hline 1850.0 & 610.0 & $09 / 69$ & $11 / 69$ & $\begin{array}{l}1 \\
2 \\
3 \\
4 \\
5 \\
6 \\
7 \\
8 \\
5 \\
10 \\
11 \\
12 \\
13 \\
14 \\
15 \\
16 \\
17 \\
18 \\
15 \\
20\end{array}$ & $\begin{array}{l}11 / 0 s / 6 s \\
c 1 / 24 / 70 \\
c 3 / 02 / 70 \\
1 c / c s / 7 c \\
12 / 04 / 70 \\
c 4 / 02 / 71 \\
c E / 01 / 71 \\
c s / 0 s / 71 \\
c 4 / 01 / 72 \\
11 / 19 / 72 \\
c 2 / 0 s / 73 \\
c 4 / 14 / 73 \\
1 c / 18 / 73 \\
c \equiv / 3 c / 74 \\
1 c / 12 / 74 \\
12 / 21 / 74 \\
c 1 / 01 / 75 \\
c 2 / 03 / 75 \\
c s / 13 / 75 \\
c 3 / 22 / 76\end{array}$ & $\begin{array}{l}11 / 30 / 69 \\
02 / 03 / 70 \\
C 7 / 12 / 70 \\
1 C / 1 s / 70 \\
12 / 21 / 70 \\
C 5 / 2 \varepsilon / 71 \\
c \in / C \varepsilon / 71 \\
09 / 22 / 71 \\
06 / 14 / 72 \\
11 / 24 / 72 \\
02 / 15 / 73 \\
C 6 / 16 / 73 \\
10 / 23 / 73 \\
C 7 / 02 / 74 \\
10 / 12 / 74 \\
12 / 31 / 74 \\
01 / 05 / 75 \\
02 / 12 / 75 \\
12 / 04 / 75 \\
C 4 / 0 s / 76\end{array}$ & 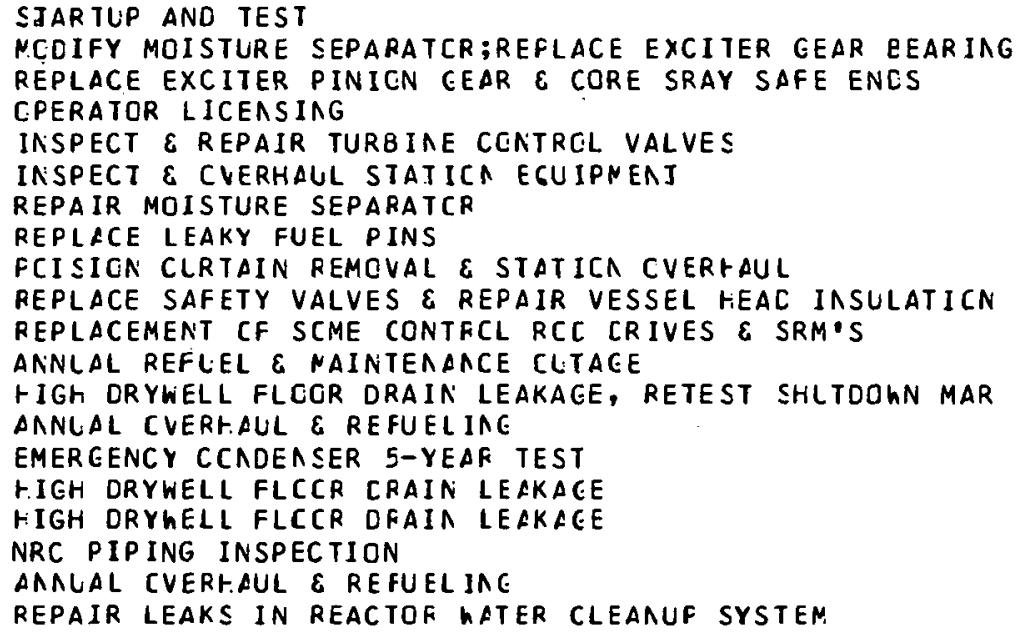 \\
\hline
\end{tabular}


CCCNEE NUCLEAR STATICA UNIT I

\begin{tabular}{|c|c|c|c|c|c|c|c|}
\hline$M W: T$ & MWE-NET & CRIT-L & ELEC TFIC & $D O W N-H$ & CINN-CATE & LP-DATE & RELSCA \\
\hline 2568.0 & 887.0 & $04 / 73$ & 05,73 & $\begin{array}{r}1 \\
2 \\
3 \\
4 \\
5 \\
6 \\
7 \\
8 \\
5 \\
10 \\
11 \\
12\end{array}$ & 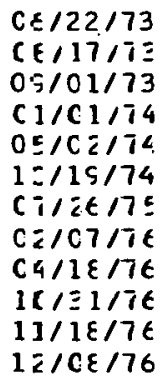 & $\begin{array}{l}07 / 09 / 73 \\
C \varepsilon / 2 ! / 73 \\
C 9 / C \subseteq / 73 \\
C 1 / 1 \varepsilon / 74 \\
C E / C \epsilon / 74 \\
C 3 / 12 / 75 \\
(E / C 1 / 75 \\
C 4 / C \epsilon / 76 \\
C 5 / 21 / 76 \\
11 / 1 \leq / 76 \\
12 / 0 \epsilon / 76 \\
12 / 20 / 76\end{array}$ & 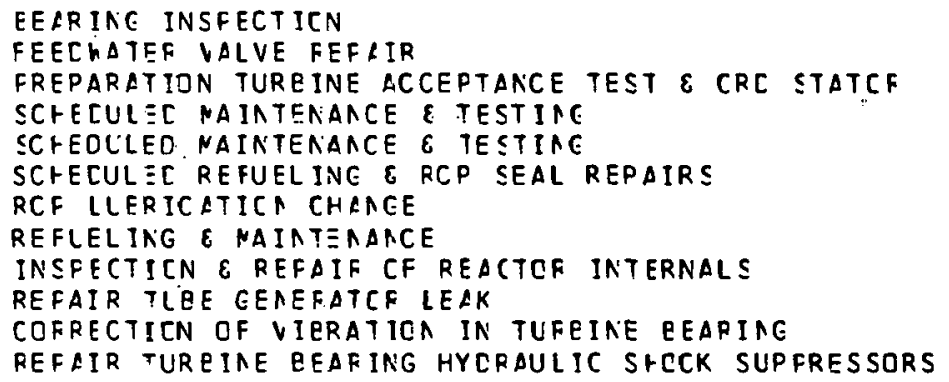 \\
\hline
\end{tabular}


CCENEE NUCLEAR STATICN UNIT 2

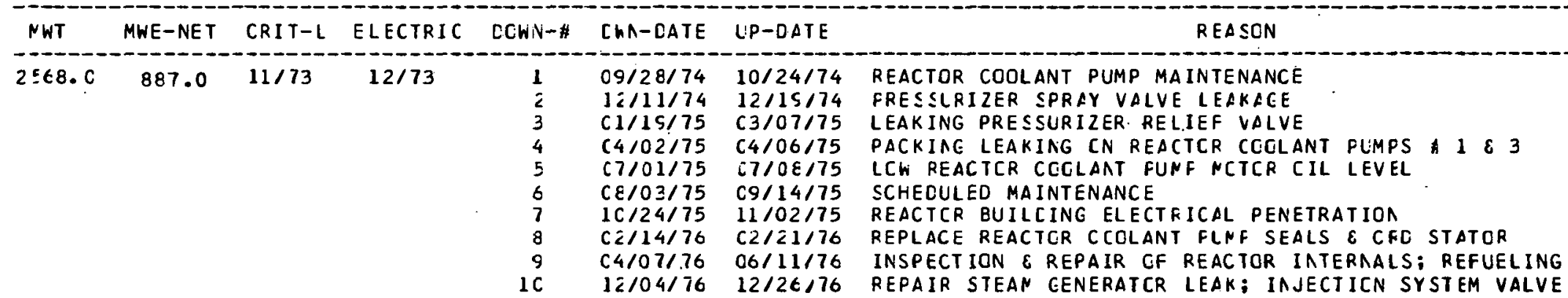


OCONEE NUCLEAR STATICA UNIT 3

\begin{tabular}{|c|c|c|c|c|c|c|c|c|}
\hline$M W T$ & MWE-NET & CRIT-L & ELECTRIC & DOWN-A & {$[n N-D A T E$} & LP-DATE & $F E L S C A$ & \\
\hline 256.8 .0 & 887.0 & $c 5 / 74$ & $05 / 74$ & $\begin{array}{r}1 \\
2 \\
3 \\
4 \\
5 \\
6 \\
7 \\
8 \\
5 \\
1 C\end{array}$ & $\begin{array}{l}12 / 23 / 74 \\
C 4 / C 7 / 75 \\
05 / 01 / 75 \\
C E / 13 / 75 \\
C 5 / 20 / 7 E \\
0 E / 23 / 76 \\
C \equiv / 20 / 76 \\
07 / C 1 / 7 \epsilon \\
C 7 / 21 / 76 \\
C ? / 1 E / 76\end{array}$ & $\begin{array}{l}C 1 / 11 / 75 \\
04 / 27 / 75 \\
05 / 14 / 75 \\
C E / 25 / 75 \\
10 / 11 / 75 \\
C 2 / 26 / 76 \\
C 4 / 17 / 76 \\
67 / C E / 76 \\
C 7 / 3 C / 76 \\
11 / 1 C / 76\end{array}$ & 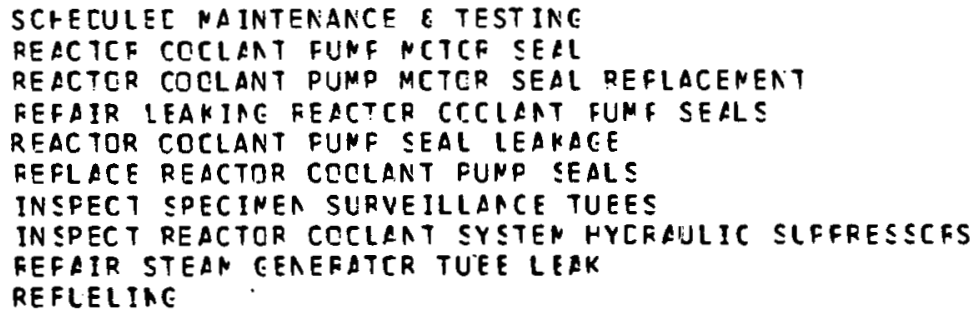 & $\ldots$ \\
\hline
\end{tabular}




\begin{tabular}{|c|c|c|c|c|c|c|c|}
\hline N.WT & MWE-NET & CR I T $T-L$ & ELECTRIC & $D C W N-\#$ & ChN-CATE & $L P-D A T E$ & REASON \\
\hline 1530.0 & 650.0 & $C 5 / 69$ & $05 / 69$ & $\begin{array}{l}1 \\
2 \\
3 \\
4 \\
5 \\
6 \\
7 \\
8 \\
9 \\
10 \\
11 \\
12 \\
12 \\
14 \\
15 \\
16 \\
17 \\
18 \\
19 \\
20 \\
21\end{array}$ & $\begin{array}{l}0 \in / 01 / 69 \\
C 2 / 01 / 70 \\
C 4 / 19 / 70 \\
10 / 17 / 70 \\
C 2 / 13 / 71 \\
C S / 18 / 71 \\
11 / 16 / 71 \\
C 5 / 02 / 72 \\
C 8 / 10 / 72 \\
C 1 / 01 / 73 \\
C 4 / 14 / 73 \\
C S / 08 / 73 \\
C 1 / 12 / 74 \\
C 3 / 07 / 74 \\
C 4 / 13 / 74 \\
1 C / 08 / 74 \\
C 2 / 04 / 75 \\
C 3 / 1 S / 75 \\
C S / 24 / 75 \\
11 / 25 / 75 \\
12 / 27 / 75\end{array}$ & $\begin{array}{l}06 / 05 / 69 \\
C 2 / 12 / 70 \\
04 / 21 / 70 \\
10 / 27 / 70 \\
c 2 / 18 / 71 \\
11 / 12 / 71 \\
11 / 22 / 71 \\
66 / 15 / 72 \\
08 / 14 / 72 \\
01 / 13 / 73 \\
C 6 / C 4 / 73 \\
10 / 05 / 73 \\
c 1 / 21 / 74 \\
C 3 / 1 / 7 / 74 \\
C 7 / 02 / 74 \\
10 / 16 / 74 \\
02 / 10 / 75 \\
05 / 26 / 75 \\
10 / 03 / 75 \\
12 / 01 / 75 \\
c 3 / 16 / 76\end{array}$ & 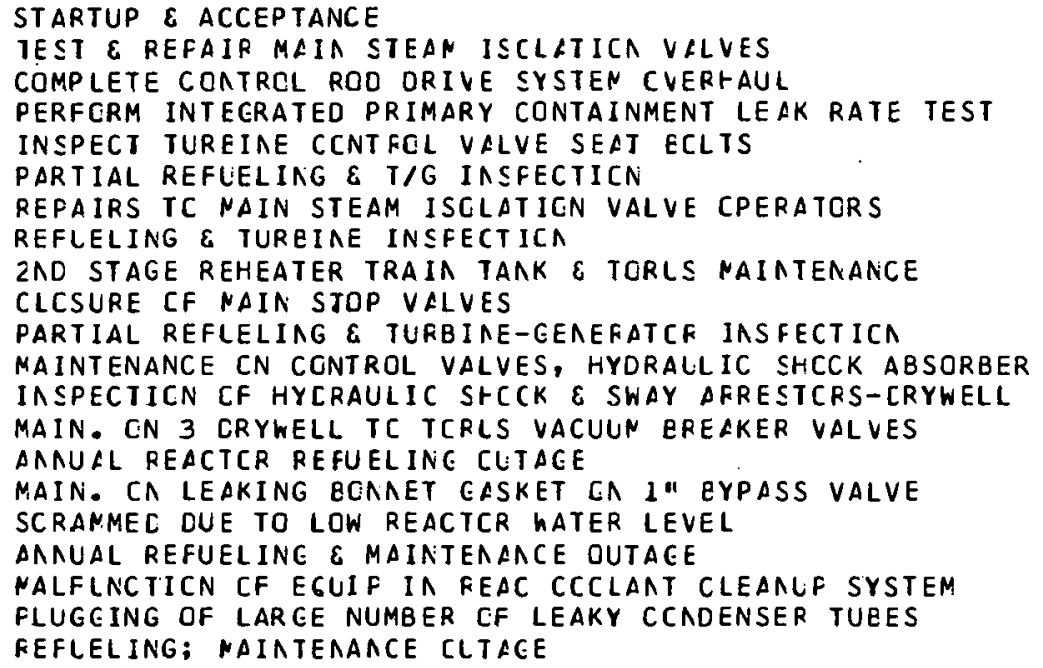 \\
\hline
\end{tabular}


PAL ISALES

\begin{tabular}{|c|c|c|c|c|c|c|c|}
\hline MW T & MWE-NE T & CPI T-L & ELECTRIC & $C C h N-A$ & {$[h A-C \angle T E$} & UF-CATE & RE $\triangle S C A$. \\
\hline 2212.0 & 668.0 & $05 / 71$ & $12 / 71$ & $\begin{array}{r}1 \\
2 \\
3 \\
4 \\
5 \\
6 \\
7 \\
8 \\
5 \\
10\end{array}$ & $\begin{array}{l}C E / 24 / 71 \\
02 / C 3 / 72 \\
C \equiv / 1 \leq / 72 \\
0</<2 / 72 \\
C E / 26 / 72 \\
C(12 / 72 \\
1 C / 23 / 72 \\
C 1 / 1 \in / 73 \\
C E / 18 / 73 \\
0 \& / 11 / 73\end{array}$ & $\begin{array}{l}C 1 / 11 / 72 \\
C 3 / 05 / 72 \\
C 3 / 24 / 72 \\
C E / C 3 / 72 \\
C 9 / 03 / 72 \\
C \subseteq / 23 / 72 \\
11 / 12 / 72 \\
C 3 / C 6 / 73 \\
C 5 / 3 C / 73 \\
10 / C 1 / 74\end{array}$ & 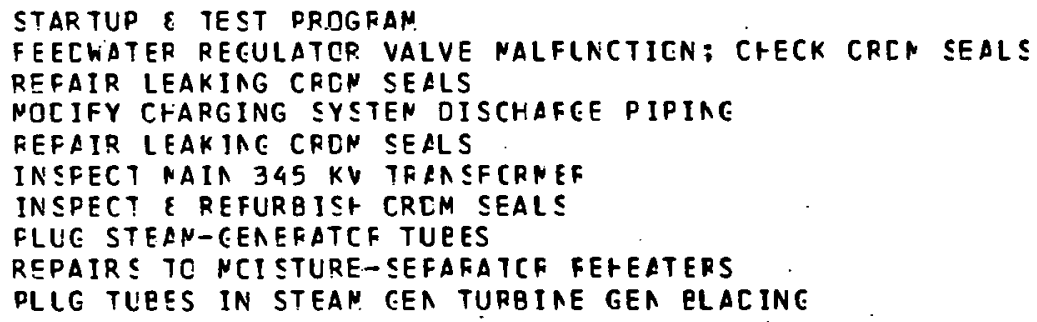 \\
\hline & & & & $\begin{array}{l}11 \\
12 \\
13 \\
14 \\
15 \\
16 \\
17\end{array}$ & 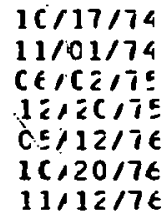 & $\begin{array}{l}1 C / 27 / 74 \\
C 4 / C 2 / 75 \\
C E / 2 C / 75 \\
C 4 / 2 \epsilon / 76 \\
C 5 / 17 / 76 \\
10 / 2 \epsilon / 76 \\
11 / 16 / 76\end{array}$ & $\begin{array}{l}\text { AEC LICEASING EXAM, CFCH FEFAIF E WATER CHER. FFCELEM } \\
\text { REPL CCNC TLEING: RPR TURBIAE; REFL MAIN CCNC TUEES } \\
\text { REFAIR LEAKINE CFCN SESLS } \\
\text { REFUELIAG } \\
\text { REPAIR CRD SEAL LEAKACE } \\
\text { REFAIR LEAK IN CHEN E VOLUNE CCNTRCL SYSTEN } \\
\text { REFAIR CENTFCL RCO CFIVE }\end{array}$ \\
\hline
\end{tabular}


PEACH BOTTOM ATOMIC POWER STATICN UNIT $\bar{z}$

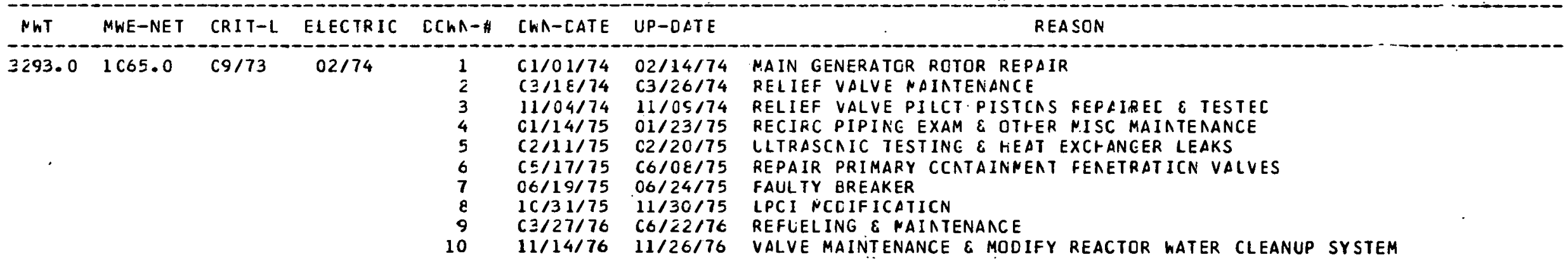


PEACH BCTTCM ATOMIC PCWER STATION UNIT

\begin{tabular}{|c|c|c|c|c|c|c|c|}
\hline MHT & MW'E-NET & CR IT-L & ELECTRIC & DCWN-A & $\operatorname{ChN} N-\operatorname{CATE}$ & $L P-D A] E$ & REASCN \\
\hline 3293.0 & 1065.0 & $C 8 / 74$ & $09 / 74$ & $\begin{array}{l}1 \\
2 \\
3 \\
4 \\
5 \\
6 \\
7 \\
8 \\
9 \\
10 \\
11 \\
12\end{array}$ & $\begin{array}{l}C S / 1 \varepsilon / 74 \\
1 / 106 / 74 \\
C 1 / 03 / 75 \\
C 1 / 17 / 75 \\
C 2 / 17 / 75 \\
C S / 17 / 75 \\
C 1 / 01 / 76 \\
C 2 / 27 / 76 \\
05 / 08 / 76 \\
C 5 / 25 / 76 \\
07 / 2 C / 76 \\
12 / 24 / 76\end{array}$ & $\begin{array}{c}10 / 03 / 74 \\
12 / 10 / 74 \\
01 / 11 / 75 \\
02 / 06 / 75 \\
02 / 23 / 75 \\
09 / 23 / 75 \\
02 / 02 / 76 \\
C 3 / 05 / 76 \\
05 / 15 / 76 \\
66 / 01 / 76 \\
c 7 / 26 / 76 \\
1,1\end{array}$ & 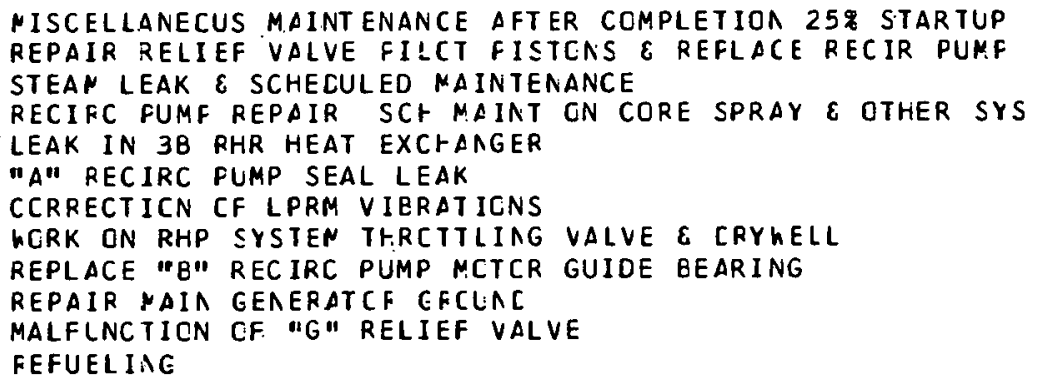 \\
\hline
\end{tabular}


PILGRIM STATICA UNIT I

\begin{tabular}{|c|c|c|c|c|c|c|c|}
\hline MHT & MWE-NET & CRIT-L & ELECTRIC & $O C H N-1$ & CHN-CATE & UP-CATE & REASON \\
\hline 1598.0 & 655.0 & $\mathrm{C} 6 / 72$ & $07 / 72$ & $\begin{array}{l}1 \\
2 \\
3 \\
4 \\
5 \\
6 \\
7 \\
8 \\
9 \\
10 \\
11 \\
12 \\
13 \\
14 \\
15\end{array}$ & $\begin{array}{l}c 7 / 24 / 72 \\
1 C / C 3 / 72 \\
11 / 29 / 72 \\
C 3 / 2 S / 73 \\
C 7 / 17 / 73 \\
C 9 / 01 / 73 \\
11 / 07 / 73 \\
12 / 28 / 73 \\
C 1 / 11 / 75 \\
C 1 / 3 C / 75 \\
C 4 / 15 / 75 \\
C 4 / 22 / 75 \\
C E / 3 C / 75 \\
C S / 13 / 75 \\
C 1 / 27 / 76\end{array}$ & $\begin{array}{l}09 / 16 / 72 \\
10 / 16 / 72 \\
12 / 07 / 72 \\
c 4 / 06 / 73 \\
07 / 30 / 73 \\
09 / 05 / 73 \\
11 / 12 / 73 \\
c 7 / 2 / 774 \\
01 / 24 / 75 \\
c 2 / 11 / 75 \\
c 4 / 21 / 75 \\
C 4 / 29 / 75 \\
c 7 / 06 / 75 \\
10 / 28 / 75 \\
C 5 / 30 / 76\end{array}$ & 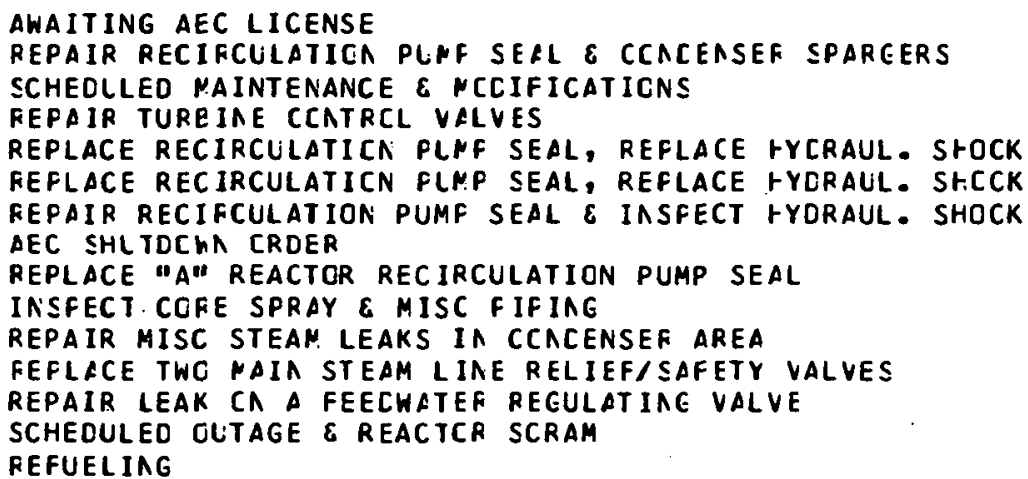 \\
\hline
\end{tabular}


PCINT BEACH NUCLEAR FIANT UNIT 1

\begin{tabular}{|c|c|c|c|c|c|c|c|}
\hline M.WT & MWF-NET & CRIT-L & ELECTRIC & CCWA $\rightarrow$ \# & CWA-CATE & UP-[ATE & REASCA. \\
\hline 1518.0 & 457.0 & $11 / 70$ & $11 / 70$ & $\begin{array}{l}1 \\
2 \\
3 \\
4 \\
5 \\
6 \\
7 \\
8\end{array}$ & $\begin{array}{l}1: / 12 / 7 C \\
C \& / C 2 / 71 \\
C</ 30 / 72 \\
C 4 / C E / 74 \\
0: / 15 / 75 \\
C: / 27 / 75 \\
11 / 16 / 75 \\
1 C / C 1 / 7 E\end{array}$ & $\begin{array}{l}11 / 21 / 70 \\
C 4 / 1 \epsilon / 71 \\
C 3 / 02 / 73 \\
C E / C 7 / 74 \\
02 / C 1 / 75 \\
04 / C E / 75 \\
C 1 / C 7 / 76 \\
11 / 24 / 76\end{array}$ & 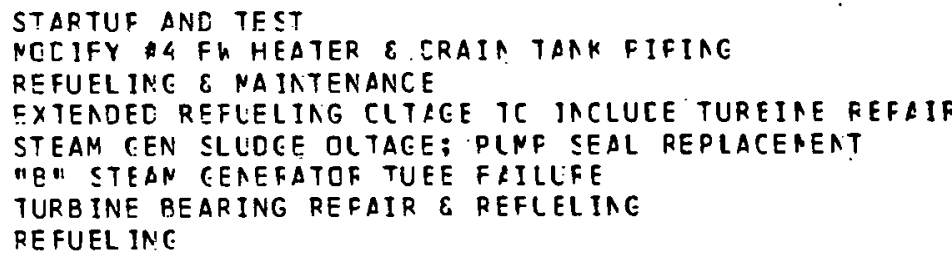 \\
\hline
\end{tabular}


FCINT EEACH NUCLEAR PLANT LNIT 2

\begin{tabular}{|c|c|c|c|c|c|c|c|}
\hline NWT & MWE-NET & CR I T - L & ELECTRIC & CCWN-\# & CИN-CATE & LP-DATE & REASON \\
\hline $1518 . \mathrm{C}$ & 497.0 & $\mathrm{C} 5 / 72$ & $08 / 72$ & $\begin{array}{l}1 \\
2 \\
3 \\
4 \\
5 \\
c \\
7\end{array}$ & $\begin{array}{l}1 C / 13 / 72 \\
(3 / 3 C / 73 \\
C 4 / 08 / 73 \\
C 5 / 03 / 73 \\
1 C / 1 \epsilon / 74 \\
C \varepsilon / 11 / 75 \\
(2 / 26 / 76\end{array}$ & $\begin{array}{l}10 / 29 / 72 \\
C 4 / 05 / 73 \\
C 4 / 13 / 73 \\
05 / 08 / 73 \\
12 / 21 / 74 \\
C 8 / 2 C / 75 \\
C 3 / 26 / 76\end{array}$ & $\begin{array}{l}\text { CCRRECT MAIN COCLANT PUMP SEALS } \\
\text { CVERFAUL CF LEAKING SAFETY VALVES } \\
\text { MACHINING OF MAIN STEAN STCF VALVE FLAFFEF STCP } \\
\text { REPLACE A PH.ASE CF MAIN STEP UP TRANSFCRMER } \\
\text { REFUELING } \\
\text { REPAIR LEAKING STEAM GENEFATCR TLEE } \\
\text { REFUELING }\end{array}$ \\
\hline
\end{tabular}


PRAIRIE ISLAND NUCLEAR GeNERATIAE. PLANT UNIT 1

\begin{tabular}{|c|c|c|c|c|c|c|c|}
\hline NWT & MWE-NET & CRIT-L & ELECTRIC & {$[C W \Lambda-\hbar$} & CWR:- CATE & UP-CATE & REA SCA \\
\hline 1650.0 & 530.0 & $12 / 73$ & $12 / 73$ & $\begin{array}{r}1 \\
2 \\
3 \\
4 \\
5 \\
6 \\
7 \\
8 \\
5 \\
10\end{array}$ & 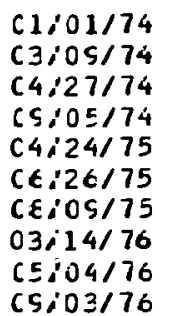 & $\begin{array}{l}02 / 06 / 74 \\
C 4 / 07 / 74 \\
C 7 / 06 / 74 \\
10 / 25 / 74 \\
05 / 14 / 75 \\
07 / 01 / 75 \\
C 8 / 17 / 75 \\
05 / 03 / 76 \\
C 5 / 10 / 76 \\
C 9 / 12 / 76\end{array}$ & $\begin{array}{l}\text { TLRBINE ELACING FAILURE } \\
\text { TURBINE ELACING FAILLRE } \\
\text { TUREINE ELACING FAILURE } \\
\text { RELACE TLRBINE } \\
\text { INSPECT STEAM GENERATOR TLBES } \\
\text { RFR LEAK IN RCP SEALINE LINE E RHR REL IEF VALVE LINE } \\
\text { REFUELINE SLRVEILLANCE E CCATAINNEAT PENETFATICN TESTIN } \\
\text { REFUEL INE } \\
\text { REPAIR INSTFLNENT THIMELE SEAL WELC } \\
\text { REPLACE REACTCR PRESSURE VESSEL LEAKING FLANGE SEALS }\end{array}$ \\
\hline
\end{tabular}


PRAIRIE ISLAND NUCLEAR GENERATING PLANT LNIT

\begin{tabular}{|c|c|c|c|c|c|c|c|}
\hline MWT & MWE-NET & CR IT-L & ELECTRIC & $O C W N-\#$ & ChN-CATE & $L P-D A T E$ & REASCN \\
\hline $1<50.0$ & 530.0 & $12 / 74$ & $12 / 74$ & $\begin{array}{l}1 \\
2 \\
3 \\
4 \\
5 \\
6\end{array}$ & $\begin{array}{l}C 3 / 08 / 75 \\
C \in / C 1 / 75 \\
C \in / 2</ 75 \\
10 / 15 / 75 \\
1</ 16 / 75 \\
1 C / 21 / 76\end{array}$ & $\begin{array}{l}03 / 15 / 75 \\
06 / 11 / 75 \\
07 / 04 / 75 \\
10 / 21 / 75 \\
01 / 15 / 76 \\
12 / 21 / 76\end{array}$ & $\begin{array}{l}\text { REPAIR HYDRCGEN COOLER LEAK \& MSIV SHAFT SEAL LEAKAGE } \\
\text { INSPECT \#5 } 8 \text { TURBINE EEARINGS FCR REFAIR } \\
\text { REPAIR FLLIC LEAK IN EH CCATRCL SYS } \& \text { FEP \# } 6 \text { EEARIAG } \\
\text { FAILURE OF } 22 \text { REACTOR CCCLANT PUNF SEALS } \\
\text { TLREINE BLACING FAILURE; STEAN GEA NCL; ELCY CUR TEST } \\
\text { REFLELING }\end{array}$ \\
\hline
\end{tabular}


GLAD-CITIES STATICN UNIT I

\begin{tabular}{|c|c|c|c|c|c|c|c|}
\hline NWT & MWE-NET & $C R I T-L$ & ELECTR IC & DONN- & [WN-CATE & LP-DATE & REASCN \\
\hline $2 \leqslant 11 . \mathrm{C}$ & 789.0 & $10 / 71$ & $c 4 / 72$ & $\begin{array}{r}1 \\
2 \\
3 \\
4 \\
5 \\
6 \\
7 \\
8 \\
9 \\
10 \\
11 \\
12 \\
13 \\
14\end{array}$ & 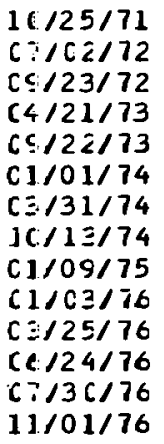 & $\begin{array}{l}04 / 12 / 72 \\
07 / 10 / 72 \\
09 / 29 / 72 \\
C 5 / 03 / 73 \\
65 / 30 / 73 \\
C 1 / 07 / 74 \\
c 7 / 21 / 74 \\
10 / 18 / 74 \\
02 / 24 / 75 \\
63 / 13 / 76 \\
64 / 05 / 76 \\
07 / 14 / 76 \\
08 / 06 / 76 \\
11 / 05 / 76\end{array}$ & 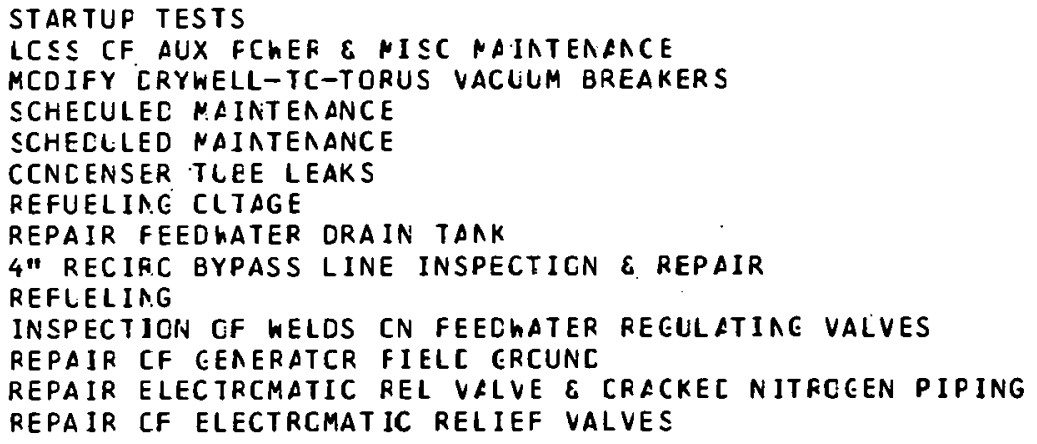 \\
\hline
\end{tabular}




\begin{tabular}{|c|c|c|c|c|c|c|c|}
\hline MHT & MWE-NET & CRIT-L & ELECTRIC & {$[C W A-A$} & CWN-CATE & UP-DATE & REA SEN \\
\hline 2511.0 & 789.0 & $04 / 72$ & $05 / 72$ & $\begin{array}{r}1 \\
2 \\
3 \\
4 \\
5 \\
6 \\
7 \\
6 \\
9 \\
10\end{array}$ & $\begin{array}{l}C 7 / 16 / 72 \\
C \varepsilon / 21 / 72 \\
C \in / 1 C / 74 \\
C \in / 18 / 74 \\
C 8 / 31 / 74 \\
(C / 12 / 74 \\
C 1 / 01 / 75 \\
C \varepsilon / 15 / 75 \\
1 C / 04 / 75 \\
C S / 1 C / 76\end{array}$ & $\begin{array}{l}07 / 24 / 72 \\
10 / 15 / 72 \\
06 / 17 / 74 \\
C 6 / 24 / 74 \\
09 / 07 / 74 \\
65 / 25 / 74 \\
04 / 3 C / 75 \\
08 / 25 / 75 \\
11 / 07 / 75 \\
11 / 04 / 76\end{array}$ & $\begin{array}{l}\text { REPAIR CABLE TRAY DAMAGE CALSEC EY FIRE } \\
\text { FEPAIR JET FUMP } 17 \\
\text { HIAIMUN FEECWATER FLCW LINE REPAIR } \\
\text { REPAIR SEAL LEAK ON CHECK VALVE 2-1201-81 } \\
\text { REPLACED RECUCERS ON LOW FLCW VALVES } \\
\text { 4-INCH RECIFCULATICN BYFASS LINE FEFAIF } \\
\text { REFUELING; 4" RECIRC BYFASS LIAE REF; FW SPARGER REPL } \\
\text { FEPAIR FW NININUN. FLCW LINE } \\
\text { CGRE MAINTENANCE CUTAGE;FEFAIR 4" FECIRC EYPASS LINE } \\
\text { REFUELING }\end{array}$ \\
\hline
\end{tabular}


RANCHO SECG NUCLEAR GENERATING STATION LNIT I

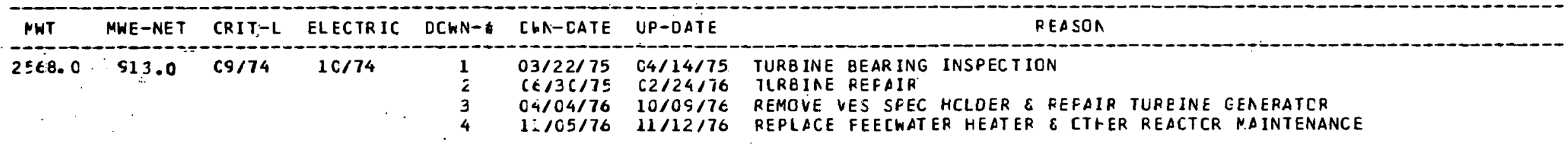


1. E ROBINSON SE PLANT UNIT Z

\begin{tabular}{|c|c|c|c|c|c|c|c|}
\hline N.WT & MHE-NET & CR I T $-L$ & ELECTRIC & $D C W N-\#$ & CHA-CATE & UP-DATE & REASON \\
\hline $\begin{array}{c}2200.0 \\
.\end{array}$ & 712.0 & $c 9 / 70$ & $09 / 70$ & $\begin{array}{l}1 \\
2 \\
3 \\
4 \\
5 \\
6 \\
7 \\
8 \\
9 \\
1 C \\
11 \\
12\end{array}$ & $\begin{array}{l}C S / 20 / 70 \\
C 3 / 14 / 71 \\
65 / 2 \varepsilon / 71 \\
C 5 / 07 / 72 \\
C 3 / 1 \epsilon / 73 \\
11 / 22 / 73 \\
05 / 06 / 74 \\
64 / 12 / 75 \\
C 5 / 02 / 75 \\
C E / 02 / 75 \\
11 / 01 / 75 \\
1 C / 3 C / 76\end{array}$ & $\begin{array}{l}12 / 31 / 70 \\
65 / 08 / 71 \\
68 / 20 / 71 \\
06 / 10 / 72 \\
05 / 14 / 73 \\
12 / C \equiv / 73 \\
06 / 24 / 74 \\
64 / 30 / 75 \\
65 / 26 / 75 \\
06 / 06 / 75 \\
12 / 16 / 75 \\
12 / 10 / 76\end{array}$ & $\begin{array}{l}\text { STARTUP E TESTING } \\
\text { TLREINE GENEFATCR EEARING FAILURE } \\
\text { TLRBINE VIBRATICN \& STEAN GENEFATCR REFAIFS } \\
\text { REPAIRS TO STEAN GENERATCR "A" } \\
\text { FIRST REFUELING CUTACE } \\
\text { STEAN GENERATCR TLBE FAILLRE } \\
\text { REFUELING, INSPECTION E REPAIR OF STEAM GENERATOR } \\
\text { STEAF CENERATCR INSPECTICA } \\
\text { REAC COOLANT PUMF SEAL FAILLRE REFAIF } \\
\text { REAC CCCLANT FUMF SEAL FAILURE REPAIR } \\
\text { REFLELING, STEAN GENERATCF INSPECTICA } \\
\text { REFUELING }\end{array}$ \\
\hline
\end{tabular}


SALEM NUCLEAR GENERATING STATIOA LNIT 1

NHT MWE-NET CRIT-L ELECTRIC DCWA-A CAN-CATE UP-CATE

REASON

335.0. C $1090.012 / 76$, 1 1 $12 / 1176$, REACTOR TESTING E INSPECTICA 


\begin{tabular}{|c|c|c|c|c|c|c|c|}
\hline NWT & MWE-NET & CRIT-L & ELECTRIC & $C C h \Lambda-\#$ & ChN-CATE & UP-OATE & REA SON \\
\hline $1 \equiv 47: c$ & 430.0 & $C 6 / 67$ & $07 / 67$ & $\begin{array}{l}1 \\
2 \\
3 \\
4 \\
5 \\
6 \\
7 \\
8 \\
9 \\
10 \\
11 \\
12 \\
13 \\
14 \\
15 \\
16 \\
17 \\
18 \\
19 \\
20 \\
21 \\
22 \\
23 \\
24 \\
25 \\
26 \\
27\end{array}$ & 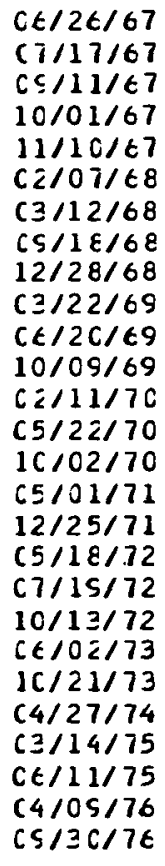 & $\begin{array}{l}07 / 07 / 67 \\
09 / 02 / 67 \\
05 / 23 / 67 \\
10 / 16 / 67 \\
12 / 03 / 67 \\
C 2 / 19 / 68 \\
09 / 12 / 68 \\
05 / 26 / 68 \\
01 / 07 / 69 \\
04 / 04 / 69 \\
08 / 05 / 69 \\
10 / 16 / 69 \\
C 2 / 15 / 70 \\
C 5 / 28 / 70 \\
11 / 19 / 70 \\
05 / 05 / 71 \\
02 / 24 / 72 \\
05 / 25 / 72 \\
C 7 / 28 / 72 \\
10 / 20 / 72 \\
67 / 27 / 73 \\
C 1 / 22 / 74 \\
c 5 / 20 / 74 \\
64 / 23 / 75 \\
06 / 16 / 75 \\
04 / 15 / 76 \\
1 / 1 / 1\end{array}$ & 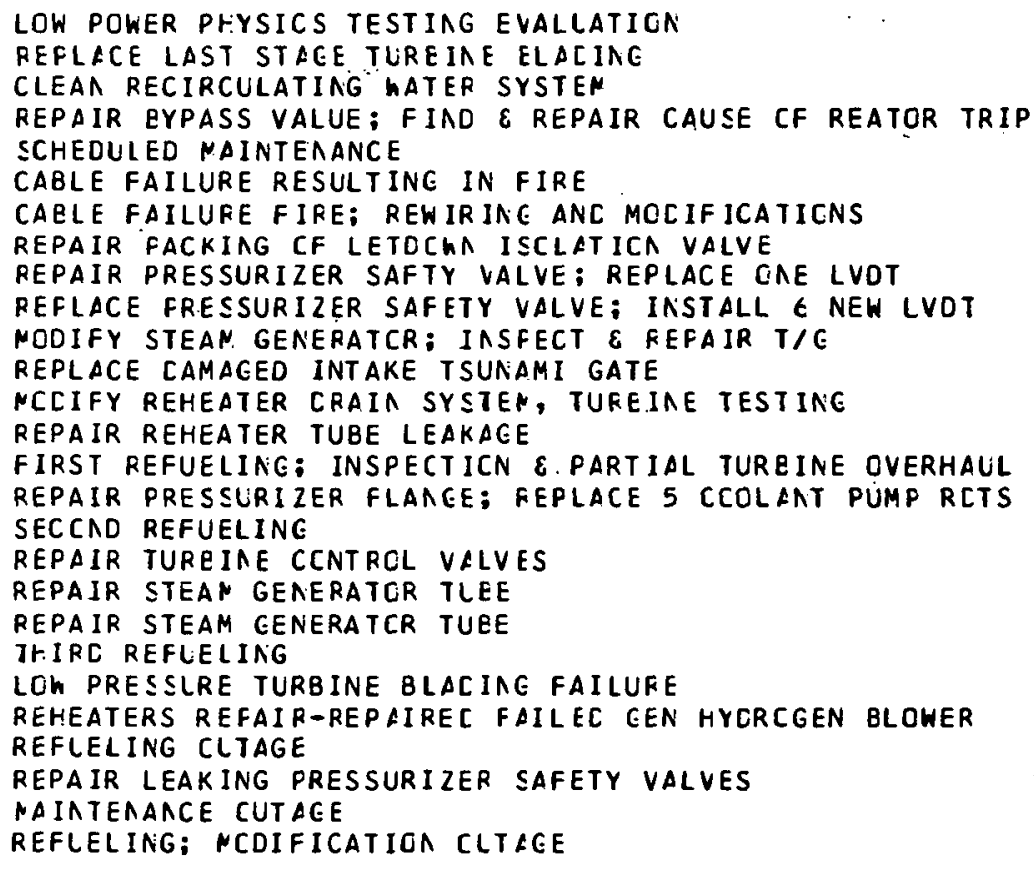 \\
\hline
\end{tabular}


ST. LUCIE UNIT 1

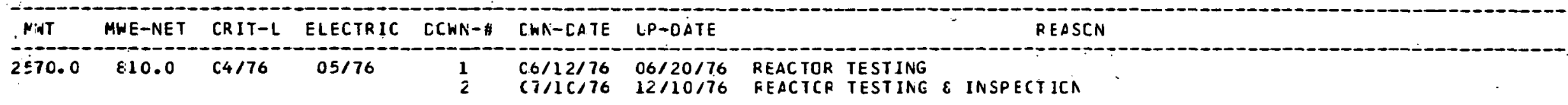


SLRRY PCWER STATICA UNIT I

\begin{tabular}{|c|c|c|c|c|c|c|c|}
\hline MWT & MWE -NET & $C R I T-L$ & ELECTR IC & $D C h N-A$ & ChN-CATE & LP-DATE & REA SON \\
\hline 2441.0 & 822.0 & $07 / 72$ & $07 / 72$ & $\begin{array}{r}1 \\
2 \\
3 \\
4 \\
5 \\
6 \\
7 \\
8 \\
9 \\
10 \\
11 \\
12 \\
13 \\
14 \\
15 \\
16 \\
17\end{array}$ & 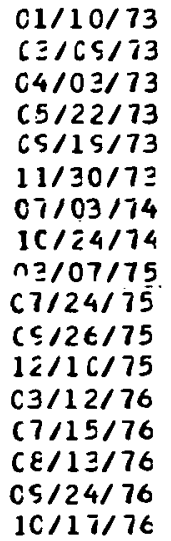 & $\begin{array}{c}01 / 29 / 73 \\
63 / 15 / 73 \\
c 4 / 11 / 73 \\
C 6 / 12 / 73 \\
10 / 24 / 73 \\
c 3 / 15 / 74 \\
C 7 / 11 / 74 \\
12 / 31 / 74 \\
C 3 / 14 / 75 \\
C 8 / 01 / 75 \\
12 / 08 / 75 \\
12 / 16 / 75 \\
03 / 18 / 76 \\
c 7 / 22 / 76 \\
C 8 / 15 / 76 \\
C 9 / 30 / 76 \\
/ 1 /\end{array}$ & 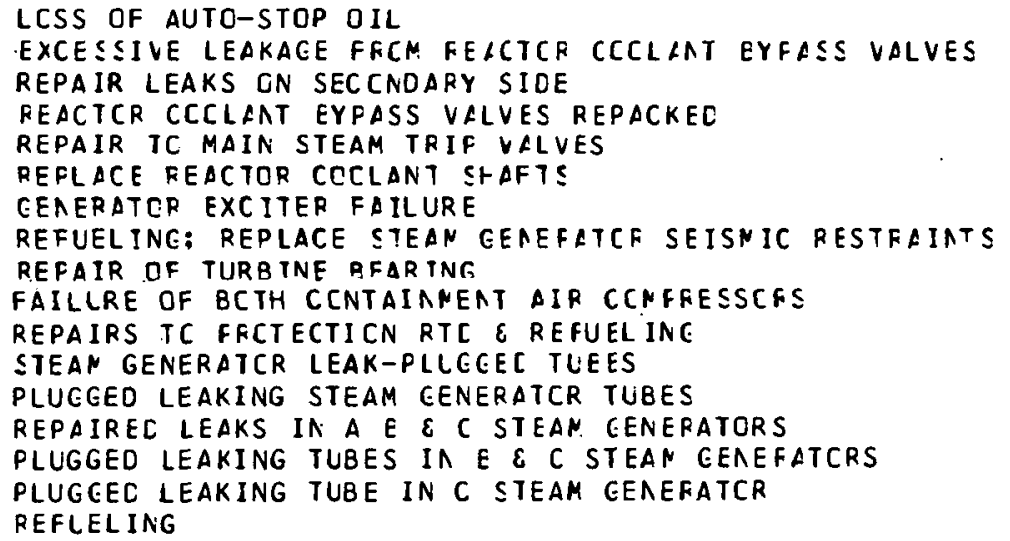 \\
\hline
\end{tabular}




\begin{tabular}{|c|c|c|c|c|c|c|c|}
\hline$N_{k}:$ & MWE-NET & CRIT-L & ELECTRIC & {$[C W n-B$} & CWN-CATE & UP-LATE & REA $\subseteq C N$ \\
\hline 2441.0 & 822.0 & $03 / 73$ & $03 / 73$ & $\begin{array}{l}1 \\
2 \\
3 \\
4 \\
5 \\
6 \\
7 \\
8 \\
9 \\
10 \\
11 \\
12\end{array}$ & $\begin{array}{l}C 5 / 07 / 73 \\
1 C / 25 / 73 \\
C 4 / 1 \equiv / 74 \\
C E / 25 / 74 \\
C C / 6 \epsilon / 74 \\
64 / 2 \epsilon / 75 \\
C 7 / C E / 75 \\
C 1 / 17 / 76 \\
C 2 / 02 / 76 \\
03 / 04 / 76 \\
C 4 / 22 / 76 \\
C S / 15 / 7 t\end{array}$ & $\begin{array}{l}C 5 / 25 / 73 \\
11 / 13 / 73 \\
66 / 07 / 74 \\
c 7 / 08 / 74 \\
12 / 31 / 74 \\
66 / 17 / 75 \\
c 7 / 11 / 75 \\
c 1 / 25 / 76 \\
62 / 11 / 76 \\
63 / 10 / 76 \\
06 / 10 / 76 \\
12 / 18 / 76\end{array}$ & 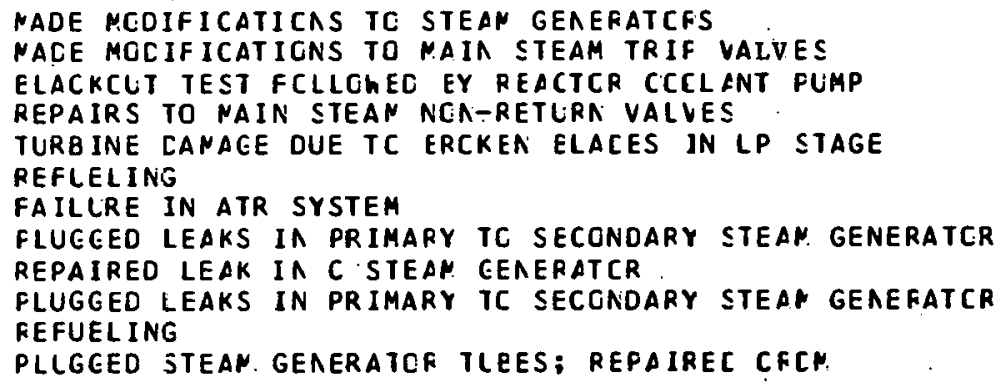 \\
\hline
\end{tabular}


THREE NILE ISLANC NUCLEAR STATICA UNIT 1

\begin{tabular}{|c|c|c|c|c|c|c|c|}
\hline NhT & $M W E-\Lambda E T$ & CRIT-L & ELECTRIC & $C(W N-\#$ & CWN- CATE & $U P-D A T E$ & REA $S C N$ \\
\hline 2535.0 & 819.0 & $06 / 74$ & $0 \in / 74$ & $\begin{array}{l}1 \\
2 \\
3 \\
4 \\
5 \\
6 \\
7 \\
8\end{array}$ & $\begin{array}{l}C E / 13 / 74 \\
1 C / 2 C / 74 \\
C 4 / 05 / 75 \\
C E / 25 / 75 \\
10 / 01 / 75 \\
11 / 12 / 75 \\
C 2 / 2 C / 76 \\
11 / 05 / 76\end{array}$ & $\begin{array}{l}08 / 23 / 74 \\
10 / 30 / 74 \\
C 4 / 13 / 75 \\
C E / 1 C / 75 \\
10 / 07 / 75 \\
11 / 24 / 75 \\
C 5 / 26 / 76 \\
12 / 04 / 76\end{array}$ & $\begin{array}{l}\text { REMCVE MESH SCREENS FRCM TLFEINE \& FEEC PLIMF STEAM LINE } \\
\text { LEAKINE RELIEF VALVES FRCN REAC. CCCLANT FRESSURIZER } \\
\text { CCATFCL RCE CROFPED.INTC CORE } \\
\text { REPAIRS \& CCATRCL RCD INTERCHANGE } \\
\text { REPAIR REACTOR COOLANT PUMP } \\
\text { FEPAIR CCNTFCL RCD CFIVE } \\
\text { REFUELING \& NAINIENANCE } \\
\text { NAINTENANCE GUTAGE }\end{array}$ \\
\hline
\end{tabular}


NHT MHE-NET CRIT-L ELECTRIC COHN-" CKN-DATE LF-CATE

RE $\&$ SCA

NWT

$3423.01130 .012 / 75$; 1 ; $119 / 7611 / 13 / 76$ REPAIR GRCUNC IN MAIN GENEFATCR 
TURKEY FCINT STATICA UNIT ?

\begin{tabular}{|c|c|c|c|c|c|c|c|}
\hline MNT. & MWE-NET & CR I T-L & ELEC TR IC & DOLN-\# & DHN-DATE & LF-DATE & REASCA \\
\hline 2200.0 & 653.0 & $10 / 72$ & $11 / 72$ & $\begin{array}{l}1 \\
2 \\
3 \\
4 \\
5 \\
6 \\
7 \\
8 \\
9 \\
10 \\
11 \\
12 \\
13 \\
14 \\
15 \\
16 \\
17 \\
18 \\
19 \\
20 \\
21\end{array}$ & 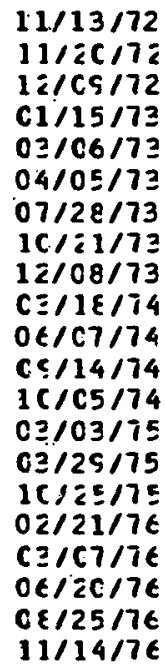 & $\begin{array}{c}11 / 17 / 72 \\
12 / 04 / 72 \\
12 / 22 / 72 \\
C 1 / 23 / 73 \\
03 / 13 / 73 \\
C 4 / 16 / 73 \\
08 / 08 / 73 \\
1 C / 2 \varepsilon / 73 \\
12 / 2 C / 73 \\
C 3 / \equiv 1 / 74 \\
C E / 13 / 74 \\
C 9 / 23 / 74 \\
12 / 15 / 74 \\
C 2 / C C / 75 \\
C 5 / 01 / 75 \\
12 / 24 / 75 \\
C 2 / 27 / 76 \\
C 2 / 12 / 76 \\
C \in / 26 / 76 \\
C 8 / 31 / 76 \\
/ / / 7\end{array}$ & 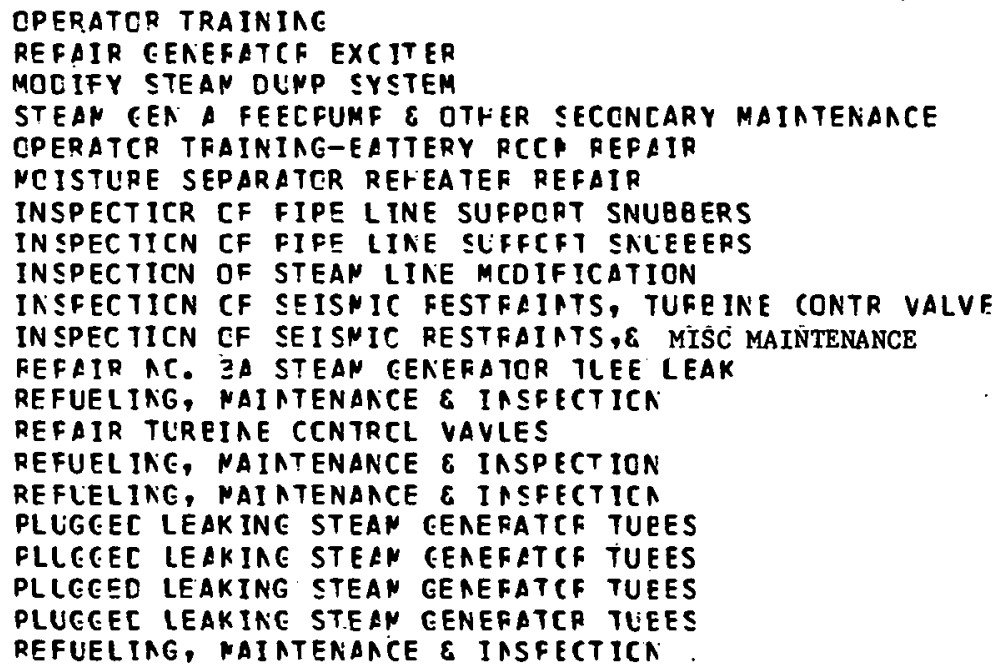 \\
\hline
\end{tabular}


TURKEY FEIAT STATIEA LNIT 4

\begin{tabular}{|c|c|c|c|c|c|c|c|}
\hline$M W T$ & MWE-NET & CRI T-L & ELECTRIC & CCWN-H & CWA-CATE & $U ! P-C \Delta T E$ & REASCA \\
\hline 2200.0 & 693.0 & $06 / 73$ & $06 / 73$ & $\begin{array}{r}1 \\
2 \\
3 \\
4 \\
5 \\
6 \\
7 \\
8 \\
5 \\
10 \\
11 \\
12 \\
13 \\
14\end{array}$ & 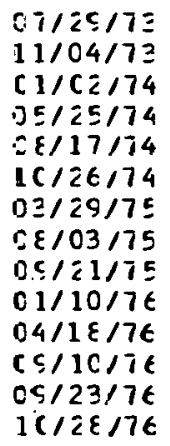 & $\begin{array}{l}C \varepsilon / C 4 / 73 \\
11 / 16 / 73 \\
C 2 / 02 / 74 \\
C E / \equiv 1 / 74 \\
C S / 10 / 74 \\
11 / 02 / 74 \\
C E / 21 / 75 \\
C 8 / C C / 75 \\
10 / 01 / 75 \\
01 / 17 / 76 \\
C 6 / 10 / 76 \\
C S / 16 / 76 \\
C 9 / 2 E / 76 \\
12 / C 2 / 76\end{array}$ & 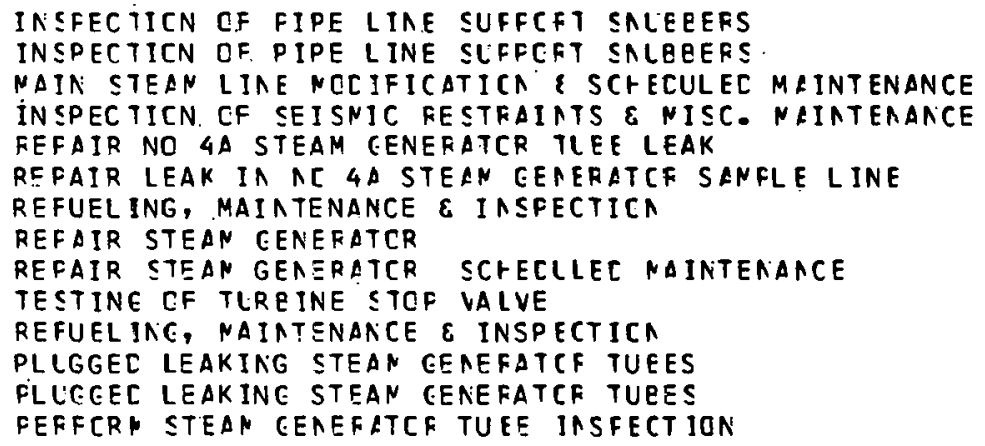 \\
\hline
\end{tabular}




\begin{tabular}{|c|c|c|c|c|c|c|c|}
\hline NWT & MWE-NET & CRIT-L & ELECTRIC & $C C h N-A$ & ChN-CATE & UP-DATE & REA SOA \\
\hline 1593.0 & 514. C & $(3 / 72$ & $0 s / 72$ & $\begin{array}{l}1 \\
2 \\
3 \\
4 \\
5 \\
6 \\
7 \\
8 \\
9 \\
10 \\
11 \\
12 \\
13\end{array}$ & $\begin{array}{l}09 / 29 / 72 \\
11 / 0 \varepsilon / 72 \\
12 / 27 / 22 \\
C 1 / 17 / 73 \\
C E / 04 / 73 \\
03 / 2 S / 74 \\
C 5 / 25 / 74 \\
1 C / 12 / 74 \\
C 6 / 05 / 75 \\
C E / 07 / 75 \\
C 1 / 27 / 76 \\
06 / 19 / 76 \\
C S / 0 E / 76\end{array}$ & $\begin{array}{l}10 / 06 / 72 \\
11 / 22 / 72 \\
01 / 10 / 73 \\
03 / 01 / 73 \\
68 / 12 / 73 \\
04 / 04 / 74 \\
06 / 05 / 74 \\
12 / 1 \equiv / 74 \\
06 / 15 / 75 \\
08 / 29 / 75 \\
c 2 / 12 / 76 \\
08 / 07 / 76 \\
09 / 15 / 76\end{array}$ & 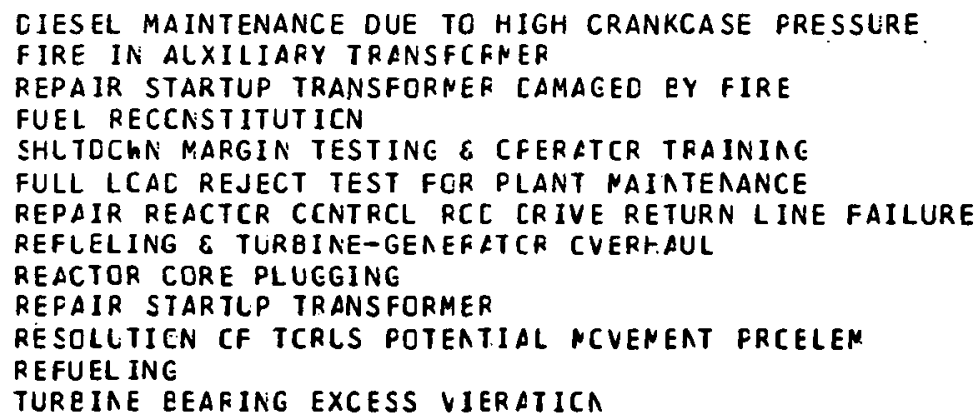 \\
\hline
\end{tabular}




\begin{tabular}{|c|c|c|c|c|c|c|c|}
\hline NWT & MWE-NET & $C R I T-L$ & ELECTRIC & COWN-A & {$[\mathrm{WN}-\mathrm{CA} T \mathrm{~T}$} & LP-DATE & FEA SCA \\
\hline $0 \in 00.0$ & 175.0 & $C 8 / 60$ & $11 / 60$ & $\begin{array}{l}1 \\
2 \\
3 \\
4 \\
5 \\
6 \\
7 \\
8 \\
9 \\
10 \\
11 \\
12 \\
12 \\
14 \\
15 \\
16 \\
17 \\
18 \\
19 \\
20 \\
21 \\
22 \\
23 \\
24 \\
25\end{array}$ & 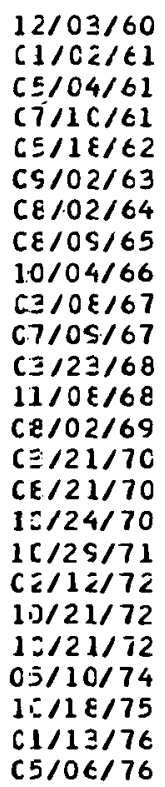 & $\begin{array}{l}12 / 13 / 60 \\
C 1 / 16 / 61 \\
05 / 09 / 61 \\
07 / 23 / 61 \\
09 / 21 / 62 \\
11 / 13 / 63 \\
C S / 06 / 64 \\
11 / 1 C / 65 \\
11 / 07 / 66 \\
63 / 20 / 67 \\
07 / 27 / 67 \\
05 / 01 / 68 \\
11 / 15 / 68 \\
09 / 25 / 69 \\
C 3 / 29 / 70 \\
C S / 01 / 70 \\
11 / 30 / 70 \\
11 / 05 / 71 \\
C 5 / 17 / 77 \\
05 / 00 / 73 \\
C 4 / C 5 / 73 \\
C 8 / 25 / 74 \\
12 / 16 / 75 \\
c 2 / 01 / 76 \\
05 / 19 / 76\end{array}$ & 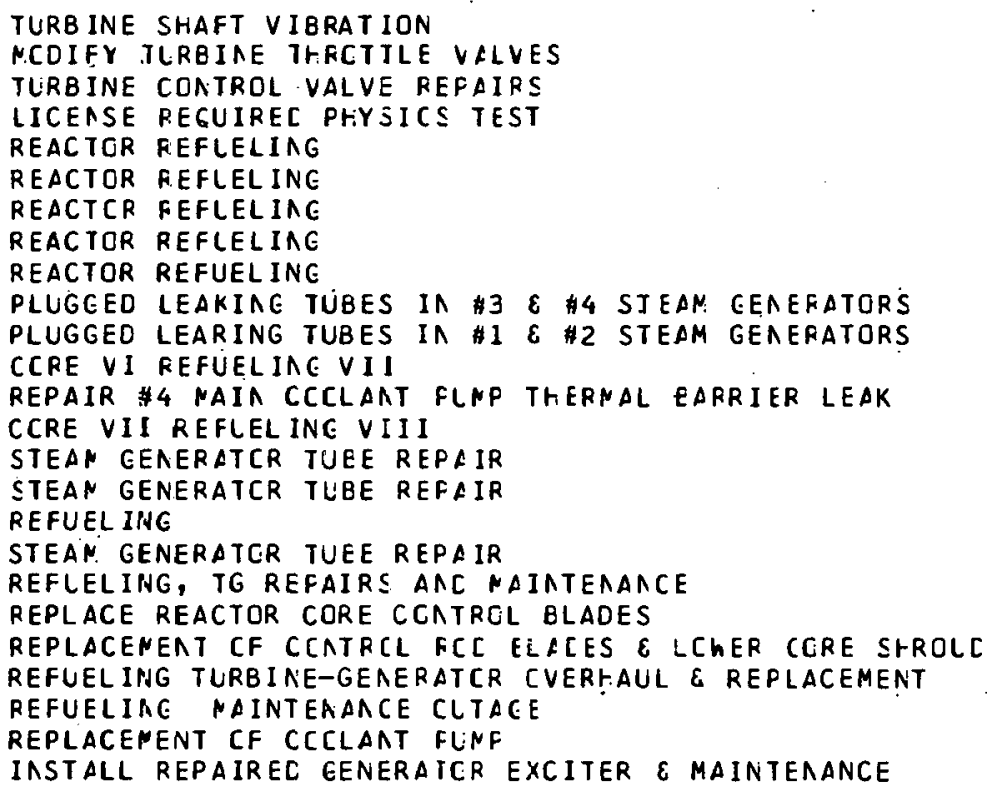 \\
\hline
\end{tabular}




\begin{tabular}{|c|c|c|c|c|c|c|c|}
\hline MH T & MWE-NET & CRI T-L & ELECTRIC & DChN-\# & {$[h N-[A] E$.} & LP- & $R E A S C A$ \\
\hline 3250.0 & 1040.0 & $06 / 73$ & $06 / 73$ & $\begin{array}{l}1 \\
2 \\
3 \\
4 \\
5 \\
6 \\
7 \\
\varepsilon \\
9 \\
10 \\
11 \\
12 \\
13 \\
14 \\
15 \\
16 \\
17\end{array}$ & 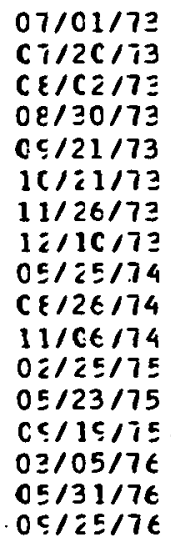 & $\begin{array}{l}C 7 / 1 E / 73 \\
C 7 / 30 / 73 \\
C 8 / 11 / 73 \\
C 9 / C C / 73 \\
10 / 01 / 73 \\
10 / 2 C / 73 \\
12 / 05 / 73 \\
C 4 / 1 \equiv / 74 \\
C E / C Z / 74 \\
C S / O 7 / 74 \\
11 / 12 / 74 \\
C 3 / 3 C / 75 \\
C 7 / 11 / 75 \\
C S / 25 / 75 \\
C 5 / 2 \epsilon / 76 \\
06 / 12 / 76 \\
C S / 2 C / 76\end{array}$ & 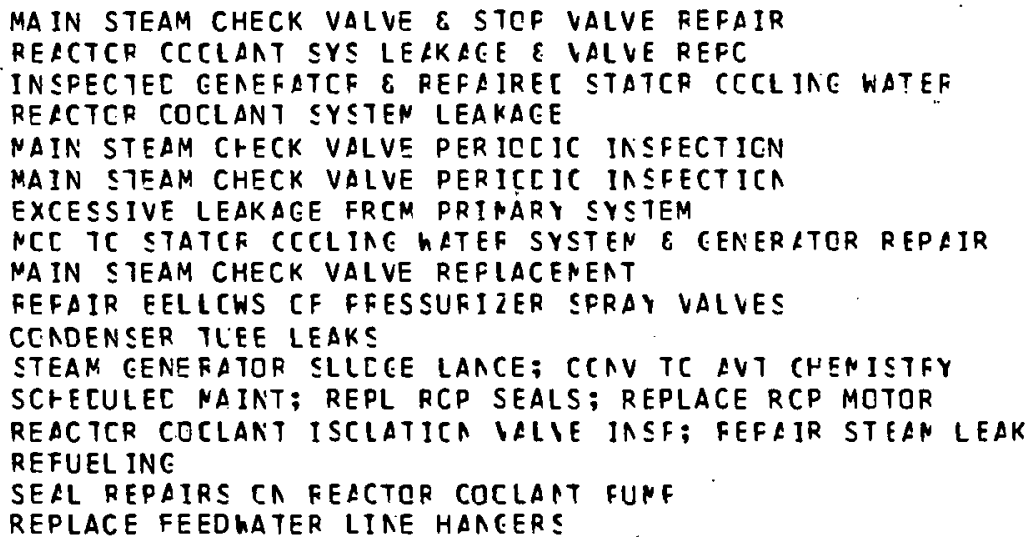 \\
\hline
\end{tabular}


Z JCN STATICN UNIT 2

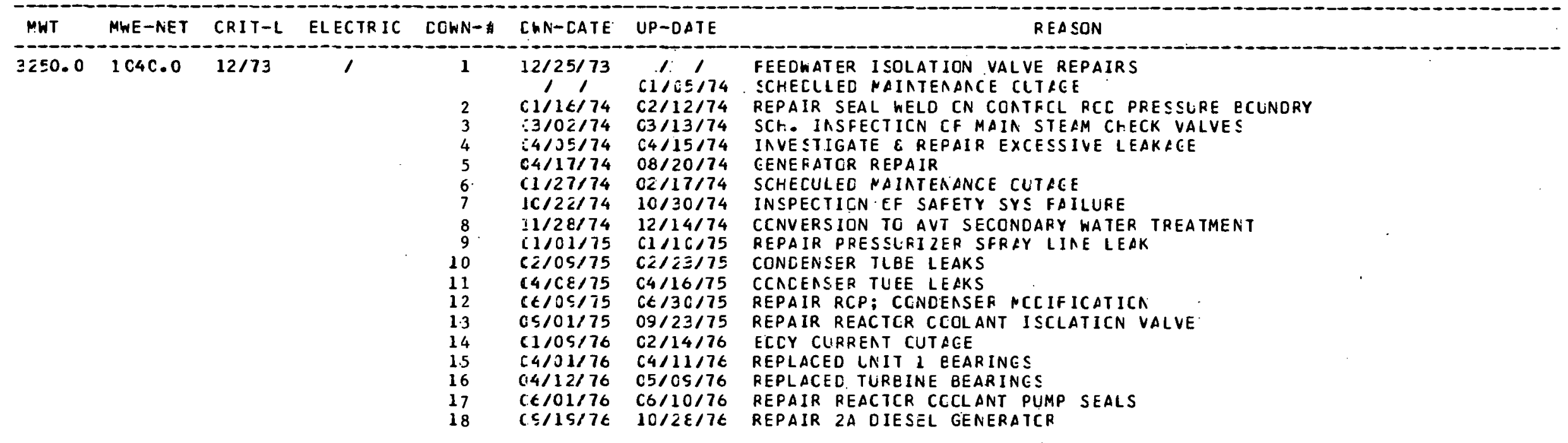




\begin{tabular}{|c|c|c|c|c|c|c|c|}
\hline NhT & MWE-NET & CRI $T-L$ & ELECTRIC & CCWN-\# & CWN- CATE & UP-DATE & REASON \\
\hline 4000.0 & 850.0 & $12 / 63$ & $04 / 66$ & $\begin{array}{l}1 \\
2 \\
3 \\
4 \\
5 \\
6 \\
7 \\
8 \\
5 \\
10 \\
11 \\
12 \\
13 \\
14 \\
15 \\
16 \\
17 \\
18 \\
15 \\
20\end{array}$ & $\begin{array}{l}C E / 01 / 66 \\
C 7 / 08 / 66 \\
C 1 / 02 / 67 \\
C 2 / 04 / 67 \\
C 3 / 12 / 67 \\
C 4 / 18 / 67 \\
C 5 / 15 / 67 \\
C E / 02 / 67 \\
C E / 21 / 67 \\
C 7 / 01 / 67 \\
C E / 26 / 67 \\
C S / 01 / 67 \\
C 7 / 20 / 69 \\
C 5 / 24 / 70 \\
C 1 / 28 / 71 \\
03 / 04 / 72 \\
C E / 12 / 73 \\
C E / 2 \equiv / 74 \\
C E / C S / 75 \\
C 4 / 2 S / 76\end{array}$ & $\begin{array}{l}06 / 05 / 66 \\
08 / 24 / 66 \\
01 / 05 / 67 \\
02 / 1 / 67 \\
03 / 21 / 67 \\
04 / 25 / 67 \\
05 / 2 t / 67 \\
06 / 07 / 67 \\
06 / 3 c / 67 \\
07 / 1 c / 67 \\
08 / 31 / 67 \\
12 / 25 / 67 \\
09 / 05 / 69 \\
C 9 / 14 / 70 \\
c 7 / 1 / 7 / 71 \\
C 7 / 30 / 72 \\
C 8 / 65 / 73 \\
C 7 / 31 / 74 \\
C 8 / 01 / 75 \\
10 / 05 / 76\end{array}$ & 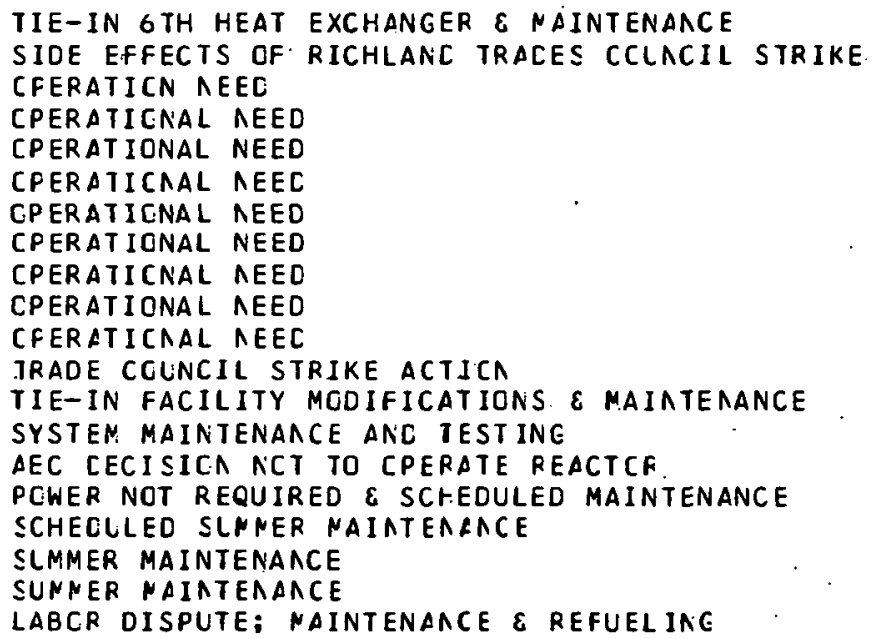 \\
\hline
\end{tabular}

